Portland State University

PDXScholar

2-10-1995

\title{
Tree Restructuring Approach to Mapping Problem in Cellular Architecture FPGAS
}

Narahari Ramineni

Portland State University

Follow this and additional works at: https://pdxscholar.library.pdx.edu/open_access_etds

Part of the Electrical and Computer Engineering Commons Let us know how access to this document benefits you.

Recommended Citation

Ramineni, Narahari, "Tree Restructuring Approach to Mapping Problem in Cellular Architecture FPGAS" (1995). Dissertations and Theses. Paper 4914.

https://doi.org/10.15760/etd.6790

This Thesis is brought to you for free and open access. It has been accepted for inclusion in Dissertations and Theses by an authorized administrator of PDXScholar. Please contact us if we can make this document more accessible: pdxscholar@pdx.edu. 


\section{THESIS APPROVAL}

The abstract and thesis of Narahari Ramineni for the Master of Science in Electrical and Computer Engineering were presented February 10, 1995, and accepted by the thesis committee and the department.

COMMITTEE APPROVALS:

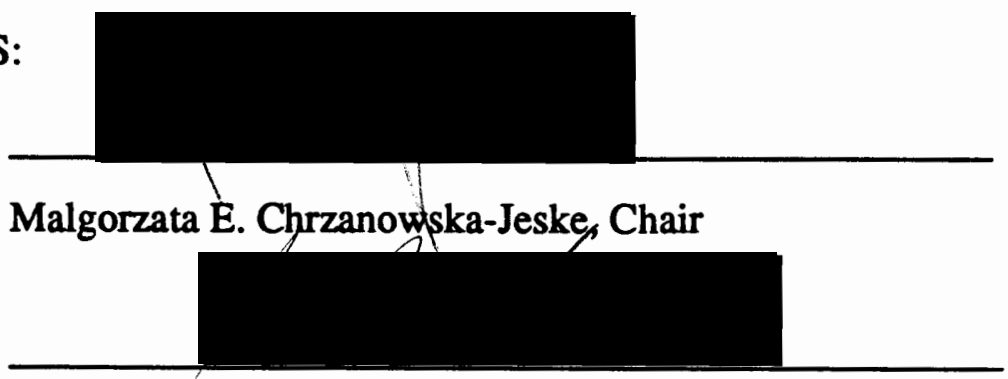

Marek A.Perkowski

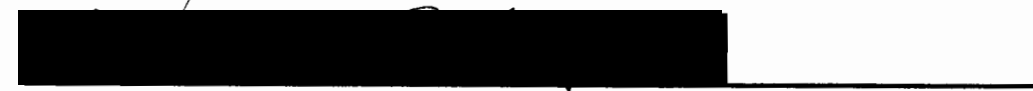

Maria Balogh

Representative of the Office of Graduate Studies

DEPARTMENT APPROVAL:

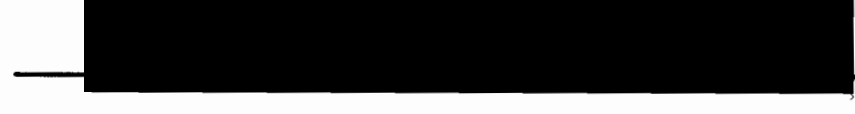

Rolf Schaumann, Chair

Department of Electrical Engineering

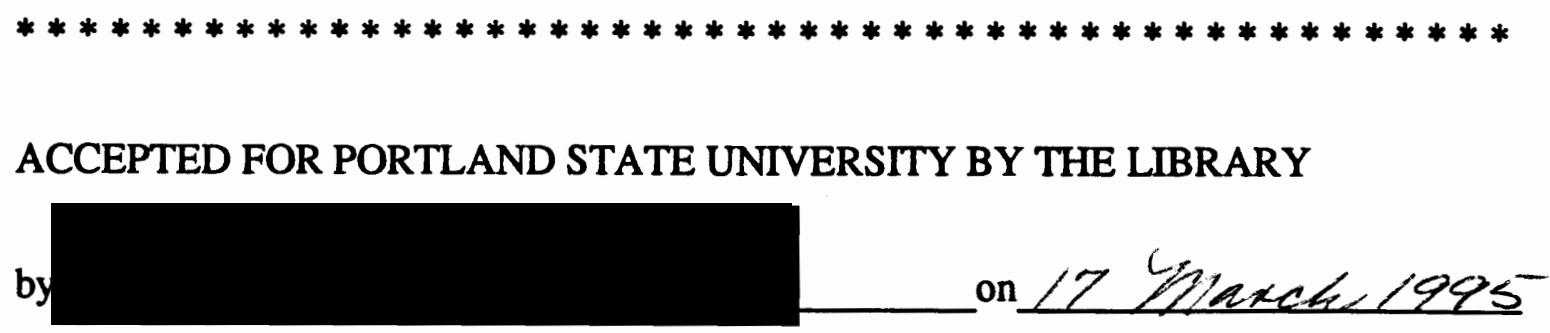




\begin{abstract}
An abstract of thesis of Narahari Ramineni for the Master of Science in Electrical and Computer Engineering presented on February 10, 1995.
\end{abstract}

Title:Tree Restructuring Approach To Mapping Problem In Cellular_Architecture FPGAS

This thesis presents a new technique for mapping combinational circuits to Fine-Grain Cellular-Architecture FPGAs. We represent the netlist as the binary tree with decision variables associated with each node of the tree. The functionality of the tree nodes is chosen based on the target FPGA architecture. The proposed tree restructuring algorithms preserve local connectivity and allow direct mapping of the trees to the cellular array, thus eliminating the traditional routing phase. Also, predictability of the signal delays is a very important advantage of the developed approach. The developed bus-assignment algorithm efficiently utilizes the medium distance routing resources (buses). The method is general and can be used for any Fine Grain CA-type FPGA. To demonstrate our techniques, ATMEL 6000 series FPGA was used as a target architecture. The area and delay comparison between our methods and commercial tools is presented using a set of MCNC benchmarks. Final layouts of the implemented designs are included. Results show that the proposed techniques outperform the available commercial tools for ATMEL 6000 FPGAs, both in area and delay optimization. 
TREE RESTRUCTURING APPROACH TO MAPPING PROBLEM IN CELLULAR_ARCHITECTURE FPGAS

by

NARAHARI RAMINENI

A thesis submitted in partial fulfillment of the requirements for the degree of

\section{MASTER OF SCIENCE \\ in \\ ELECTRICAL AND COMPUTER ENGINEERING}

Portland State University

1995 


\section{ACKNOWLEDGEMENT}

I would like to thank Dr. Malgorzata Chrzanowska-Jeske, my advisor, for providing guidance in my research work and supporting my career growth. I thank her for the methodical introduction to the latest research by organizing seminars and the reading and conference group, which had help me to gain knowledge beyond the research area. I would like to thank Dr. Marek A. Perkowski and Dr. Maria Balogh for serving on my committee and for their numerous suggestions in the preparation of the thesis.

I am grateful to Naveen Buddi for his patience in going through my thesis, papers, presentations and making valuable suggestions and corrections. A special person I want to mention here is Ajith Kumar Dasari. We have been supportive of each other in completing the academic work and building professional career. I also thank all my co-researchers for their suggestions in my thesis work.

I would like to acknowledge the travel grants I received from Academically Controlled Activities Committee at Portland State University. It was through their generous funding of my participation in 36th Midwest Symposium On Circuits \& Systems and the first publication of this thesis work was made possible.

And my special thanks go to Ms. Shirley Clark for her support through these years. I also would like to thank Laura Riddell, and all the office staff for providing the facilities and help.

This thesis work is dedicated to my mother who provided me with the best education right from my primary school and consistently encouraged and supported me to pursue higher education without which, this work would not have been possible. 
I dedicate this work to my beloved Mother Mrs. Jagadamba Ramineni 


\section{TABLE OF CONTENTS}

PAGE

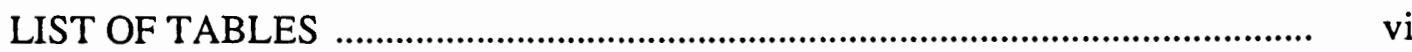

LIST OF FIGURES _................................................................................... vii

\section{CHAPTER}

I Introduction ............................................................................

II Cellular Arrays .................................................................... 7

II.1 Cellular Logic ......................................................................... 7

II.2 Generic CA-type FPGA ……………………....................... 11

III Layout-Driven Logic Synthesis ....................................................... 15

IV Architecture of ATMEL 6000 FPGA ............................................... 22

IV.1 The Symmetrical Array …….................................................. 22

IV.2 The Bussing Network ............................................................. 22

IV.3 Cell States …….............................................................. 24

IV.4 Architecture Restrictions ...................................................... 28

V Our Approach ........................................................................... 29

V.1 Tree Restructuring ................................................................. 29

V.2 Problem Formulation ………….......................................... 31

V.3 TRM Approach ............................................................... 32 
VI Description of Algorithms …................................................... 34

VI.1 Technology Mapping ...................................................... 34

VI.2 Squashed Binary Tree (SBT) ........................................... 41

VI.3 Modified Squashed Binary Tree (MSBT) ............................ 44

VI.4 Bus Assignment ............................................................ 45

VI.5 Multi-Output Functions ................................................. 48

VI.5 Comparision with Other Methods ...................................... 48

VII Results ........................................................................... 52

VIII Conclusions …................................................................... 57

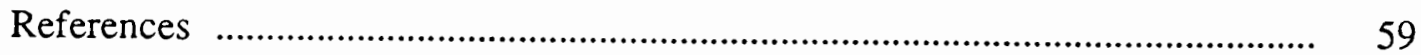

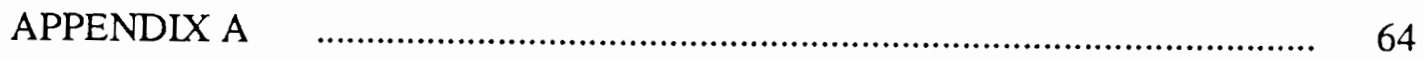

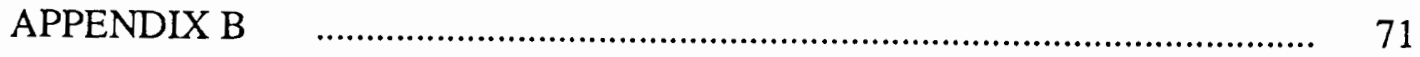




\section{LIST OF TABLES}

TABLE

PAGE

I Cell Utilization SBT Vs. MSBT

53

II

Area Comparison TRM Vs. ATMEL (IDS)

54

III

Delay Comparison TRM Vs. ATMEL (IDS)

55 


\section{LIST OF FIGURES}

$\begin{array}{ll}\text { FIGURE } & \text { PAGE }\end{array}$

1.1 Typical CAD System for FPGAs ................................................... 3

$2.1 \quad$ Maitra Cascade …........................................................................

$2.2 \quad$ Redundant Maitra Cascade …………............................................... 8

2.3 Two Rail Cascade .............................................................................

$2.4 \quad$ A Generic CA-type FPGA ……………....................................... 12

$2.5 \quad$ Logic Block of Algotronix FPGA …….............................................. 13

2.6 Logic Block of Motorola FPGA ………….................................... 14

3.1 Circuit Realization of Shannon and Davio I and II Expansion .......... 18

3.2 PRM Tree of the Boolean Function from Example 1 ........................ 21

4.1 Symetric Array of the ATMEL 6000 Chip ........................................ 23

$4.2 \quad$ Bussing Network ......................................................................... 24

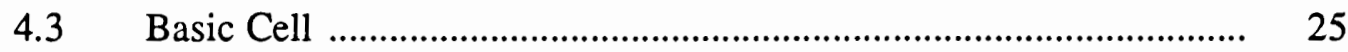

4.4 Combinatorial States .................................................................... 25

4.5 AND-EXOR Realization ............................................................... 26

4.6 Register and Constant States ............................................................... 27

4.7 Four Possible Input Configurations to a Cell ...................................... 28

5.1 Binary Tree to Squashed Binary Tree Transformation ...................... 30

$5.2 \quad$ TRM Flowgraph ........................................................................ 33

6.1 Ilustration of Type I Mismatch …………........................................ 35

$6.2 \quad$ "Switch Cell" - Solution to Type I Mismatch .................................... 36

6.3 A Two Cell Realization of the EXOR-AND Chain ............................. 37 
6.4 One Cell Realization of the EXOR-AND Chain

6.5 Grouped PRMT of the Example from Figure 3.2

6.6 A Grouped PRMT as a Binary Tree

6.7 Squashed Binary Tree (SBT)

6.8 Mapping from SBT to the Cellular Array

6.9 Critical Path Control

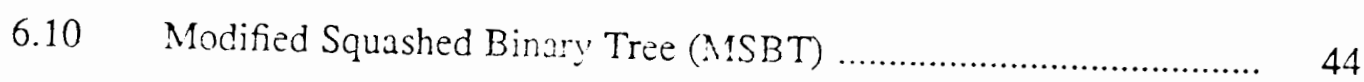

6.11 Mapping MSBT to the Cellular Array ........................................ 45

6.12 Final Mapping of MSBT with Bus Assignment .......................... 47

6.13 CMLA: Two-Dimensional Array ............................................... 49

6.14 CMLA: Realization of a Function .......................................... 51

7.1 Layout of MCNC 5x1 Benchmark Generated by ATMEL Tools ..... 56

7.2 Layout of MCNC $5 \times 1$ Benchmark Generated by TRM Package ..... 56 


\section{CHAPTER I}

\section{INTRODUCTION}

Field Programmable Gate Arrays (FPGAs) have become a popular design technology for designers seeking fast and cost effective implementations of their circuits. FPGAs are regular arrays of programmable logic blocks connected by programmable interconnections. Both, logic blocks and interconnections are programmable by the user, circuit or system designer. The available FPGA architectures differ in sizes and functionality of the logic blocks, in the floorplan of the chip, and in the structure of the routing domain. Based on these differences, FPGAs can be classified into four basic groups; Complex PLDs, Look-Up-Tables, Row-Based, and Fine-Grain CellularArchitecture FPGAs [7]. Programming technology available to program these devices are anti-fuses, static RAM cells, EPROM transistors, and EEPROM transistors. The existence of the different chip architectures combined with the fast and cheap redesigning gives the designer possibility to choose an FPGA which has the optimal features for the specific application. Therefore, FPGAs provide an excellent solution to the demands of shrinking development cycles, rapid prototyping, and applications that benefit from re-programming in field.

In recent years a lot of effort was spent on the development of the technology mapping and layout synthesis methods for the two most popular categories of these devices, namely look-up-table based (LUT-based) and row-based FPGAs. A number of the architecture-specific technology mapping approaches were developed 
$[9,10,11,37,38,39,40]$, but most of the placement and routing techniques were adopted from the semi-custom design styles like standard cells and gate arrays, with some necessary modifications. The other two types of the programmable architectures have not drawn so far much attention of the research community. In this thesis we focus on layout synthesis for one of these other categories of FPGAs, mainly Cellular-Architecture (CA) FPGAs $[2,8,13]$. The main feature of these devices, which distinguished them from the other groups is the local connectivity between logic blocks placed in a symmetrical array. Logic blocks are usually of the small granularity and of the standardcell type with a limited number of inputs and outputs. Local or global buses are used for distance connections. The method of separate technology mapping, placement and routing, used for other FPGAs, become of little value for these devices primarily due to the local connectivity. Therefore, new comprehensive methods to the layout-synthesis problem need to be developed to efficiently utilize the potential of CA-type FPGAs.

Typical steps involved to implement a circuit using programmable hardware devices are shown in Fig 1.1. If this traditional approach is used for CA-type FPGAs, a large number of logic cells is used for wiring connections or left unused [13]. This problem is mainly caused by not preserving local connectivity during the synthesis steps. Frequently, local buses need to be used to complete even very short connections, which increases circuit delay. To avoid the excessive usage of the local buses a better solution is to use different logic implementations with logic cells used as wiring cells. But this will lead to wasting a large number logic cells for wiring in the technology mapping.

The "macro block" approach which is currently used in the industry [13], to solve the layout problem for these devices is based on macro-generators. A technology independent representation of a circuit design is covered with a minimum number of 


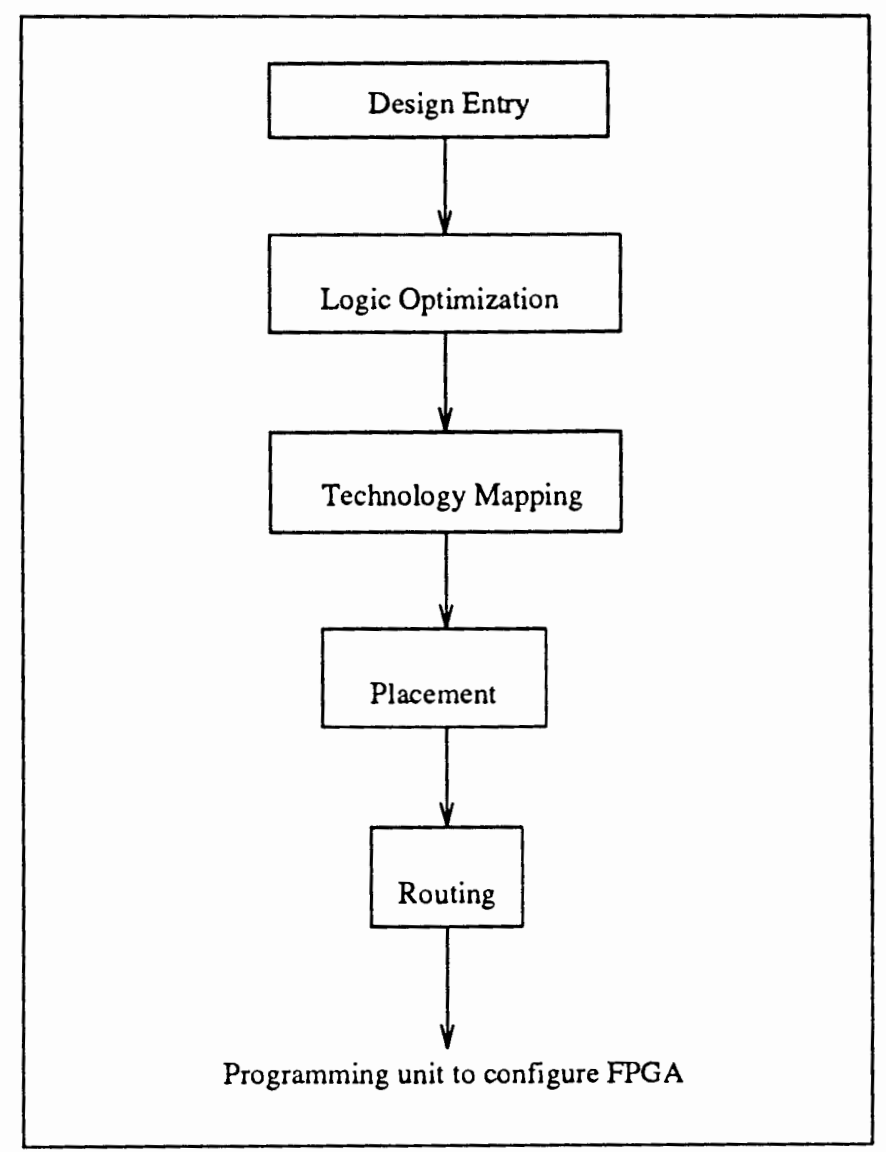

Figure 1.1 Typical CAD System for FPGAs

relatively small standard subfunctions (macros) which usually have non-uniform shapes. Placement of macros is usually performed using simulated annealing algorithm, which places the macros far from each other to assure that in the routing phase all connections between marcos can be completed. This technique leaves a lot of unused cells around the placed macros for possible use as routing blocks. Consequently, the number of cells which need to be used for routing between macros is very large. In ATMEL 6000 , series on average, about $70 \%$ of the area occupied by a design is used for wiring connections or left unused $[2,13]$. This problem is mainly caused by not creating locally connected netlist during the synthesis steps. As the routing 
resources are very limited, efficient usage of these resources can significantly reduce the area occupied by the design and thereby increase the capacity of the chip and improve circuit performance.

Recently, several logic synthesis approaches applicable to CA-type FPGAs have been presented $[1,11,17,43,44]$. In order to create a circuit layout some of these methods require an additional, separate layout synthesis step and some others do not. For example, if logic functions are represented as a two-dimensional array, they can be directly mapped onto a given CA-type architecture. The spectral methods based on orthogonal expansions [17] and Universal XOR Forms, [16] and restricted factorization method [11] based on the classical cellular arrays [15] belong to the latter type. While the spectral methods are more general and usually lead to a better solution, the algebraic one leads to much more efficient algorithms. The approaches $[1,6,12,17]$ based on trees and decision diagrams [14] which preserve well local connectivity have also been reported. In most cases, however, when the tree is finally mapped to a rectangular area, the triangular structure of the tree may waste a large amount of area. Therefore, new comprehensive solutions to the optimized mapping of such trees to the regular, locally connected arrays are of interest.

Among new approaches to the logic synthesis for CA-type FPGAs recently introduced decision diagrams are based on the decomposition of Boolean functions using combinations of Shannon and two Davio expansions. Function representations based on EXOR gates are especially attractive for CA-type FPGA, because EXOR gates are usually available in logic blocks of these devices. The cost of these EXOR gates is comparable with other gates, because fan-in is usually low. In addition, EXOR gate based representations usually give the more compact implementation of the function. These various decision diagrams have structure of the binary tree with decomposition 
variables associated with each node of the tree.

In this thesis, we propose a new approach to the mapping of the above mentioned decision diagrams onto the FPGAs with localized connections, like the CA-type FPGAs. The approach is based on restructuring of the binary tree before final mapping is performed. A Squashed Binary Tree (SBT) [3] and a new Modified Squashed Binary Tree (MSBT) approaches are used to restructure a binary tree, such that when it is mapped to the CA-type FPGA it will result in a rectangular shape rather than triangular shape of the occupied area. The method developed here is applicable to any general binary tree. To illustrate our approach, we present results for the input represented as Permuted Reed-Muller Tree (PRMT), which is obtained by applying Davio I Expansion [14] to a Boolean function. Our general approach is presented using ATMEL 6000 Series of FPGAs as the target architecture, but it has to be underlined that our approach, can be adopted to other CA-type FPGAs, such as Motorola, Algotronix or Pilkington. Algorithms are written in C and implemented on SPARC 10 workstation.

The thesis is organized as follows. In Chapter II we present a generic model of the CA-type FPGA and discuss research on cellular arrays. Chapter III describes the logic synthesis methods to generate various binary decision diagrams especially Permuted Reed-Muller Tree and its relation to the architecture of the CA-type FPGAs. In Chapter IV architecture and restrictions of the ATMEL 6000 FPGA are discussed. Our general Tree Restructuring Method (TRM) approach based on the Squashed Binary Tree (SBT) algorithm together with formulation of the problem are presented in Chapter V. Chapter VI discusses the solution methods to the tree restructuring problem. The new solution method introduced by the author i.e. Modified SBT (MSBT) approach will be discussed along with Bus-Assignment algorithm. We also compare our method to the other methods being currently developed for CA-type FPGAs. 
Results comparing SBT and MSBT approaches are given in Chapter VII to show that our approach gives better results both in area and delay optimization compared to the ATMEL IDS tools for all examined designs. Conclusions from our research are given in Chapter VIII. 


\section{CHAPTER II}

\section{CELLULAR-ARRAYS}

In this chapter we describe a generic CA-type FPGA. The local connectivity among the logic blocks makes these FPGAs to resemble cellular arrays which were studied in the sixties and seventies. In Section 2.1 the methods to realize digital logic in cellular arrays will be described. The current research in logic synthesis [11,12] for these new CA-type FPGAs are an extension to the methods developed for cellular logic. In Section 2.2 we present a generic model of the CA-type FPGA.

\subsection{Cellular Logic}

Cellular logic deals with mathematical models as well as synthesis and analysis techniques of digital networks in cellular array. "A Cellular array is a 1-, 2-, or 3dimensional iterative arrangement of similar or identical logic cells with a uniform interconnection pattern on the cells" [32]. Synthesis methods for cellular arrays, namely the Maitra cascades [27], Two-Rail cascades [24], Cutpoint cellular logic [28] have been developed and studied, however they were never used in any practical design $[24,26,27,28,29,30,31,32,33,34,36]$.

The cellular arrays can be classified into simple one-dimensional cellular arrays, "Multi-rail cascades", and two-dimensional arrays. The simple one-dimensional arrays are also known as Maitra Cascades [27]. A Maitra Cascades is a one-dimensional array of 2-input, 1-output binary combinational cells and is shown in Fig. 2.1. The veritcal input is connected to a binary 0 or 1 , while the horizontal inputs $\mathrm{x} 1, \mathrm{x} 2, \ldots, \mathrm{xn}$ are 


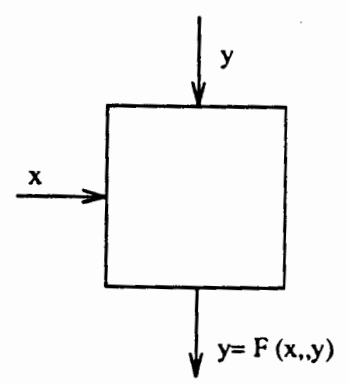

Figure 2.1 Maitra Cascade

input variables. In this cascade, each cell is capable of producing any one of the sixteen possible binary functions of two inputs. It was shown, however, by Stone and Korenjak [34] that even using redundant cascades, in which certain vertical inputs are connected to more than one cell, not all functions are realizable in this cellular array. Redundant Maitra cascade is shown in Fig. 2.2.

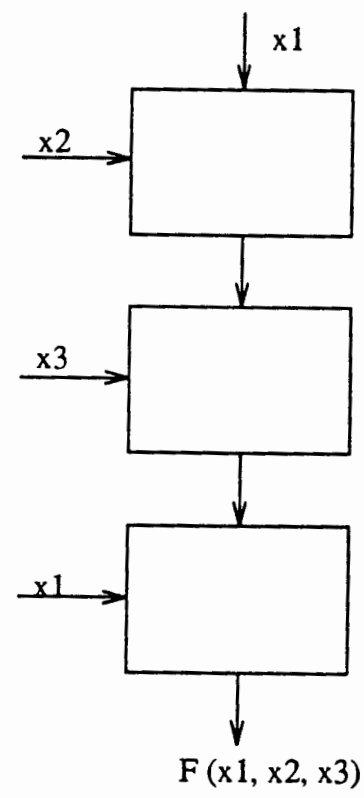

Figure 2.2 Redundant Maitra Cascade

The same deficiency exist for generalized Maitra cascades [31] which are used to realize multi-valued functions. 
One attempt to overcome the logical incompleteness of simple one-dimensional arrays is the two-rail cascades method. Short [33] has shown that every binary function is realizable by means of 3-input, 2-output cells, as shown in Fig. 2.3 .

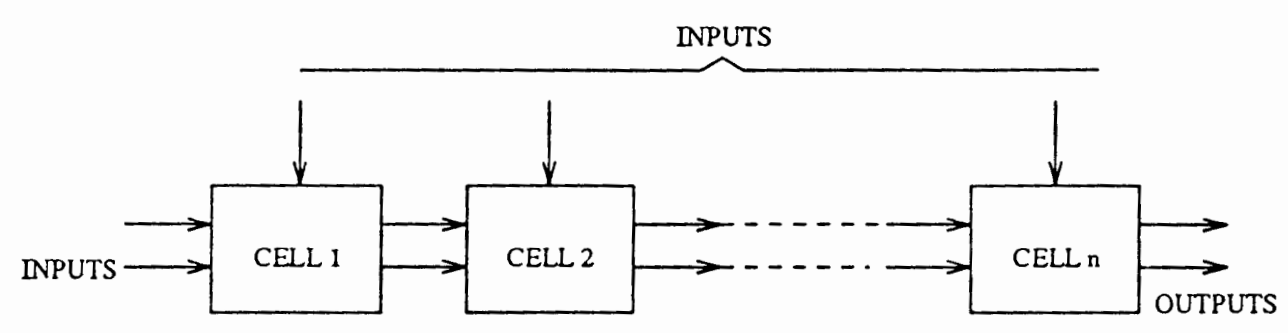

Figure 2.3 Two Rail Cascade

To realize a single binary function in the synthesis methods developed by Short, only one of the final outputs was required. Yoeli and Turner [36] extended the treatment of two-rail cascades to both output signals and showed that two-rail cascades are functionally complete for realizing an arbitrary pair of Boolean functions of any number of variables. Among the synthesis methods for two-rail cascades, four major approaches can be noted. These approaches are the ones introduced by Short [33], Yoeli [36], Elspas [25] which generalizes Yoeli, and Dvorak [24]. An extension to tworail cascades is the multi-rail cascades. Here, instead of two rails, the cells are assumed to have more than two horizontal inputs and outputs. The disadvantage of the multi-rail cascades is their serial structure, which makes them slow, the methods developed were not feasible as the number of cells required to realize the functions had exponential growth with the increase in the number of variables.

Two-dimensional arrays $[27,28,29,30]$ provide another attempt to overcome the limitation of Maitra cascades. These arrays realize a two-level representation of a function where each minterm is realized in one column of the arrays and the outputs are 
ORed in a row (called the collector row) of gates to the Maitra cascade columns. This approach was mostly used for two-level Sum of Products and positive polarity AND/XOR representations rather than the more general multi-level ones. It was shown by Minnick [29] that an arbitrary function of $n$ variables can be realized with no more than $(n+1) 2^{n-2}$ cells with the two dimensional approach.

Some multi-level representations geared towards specialized cellular arrays. One such example are the functionally complete cutpoint arrays [28]. These arrays are composed of columns of Maitra cascades where each cell needs to realize only six possible functions of two input variable. The "cutpoint" in this array refers to the specification bits in each cell to program the type of the operation it will be performing. The main deficiency of this architecture is the large number of cells that do not perform any actual function. Furthermore, there exist no communication between the horizontal and vertical inputs. Hence if a signal needs to be connected to both horizontal and vertical inputs then it results in unneccessary logic duplication.

Another attempt at multi-level representation of the functions was the Unate Cellular Logic by Mukhopadhyay [31]. In this approach the cells are assumed to be unate two-input functions, i.e. all functions except XOR and XNOR. Each cascade in this array can realize a unate function and the whole array is considered to be a twodimensional arrangement of the unate cascades. In the synthesis method, a test for unate cascade realizability is provided.

Minnick introduced the cobweb array [29] where the basic structure of the cutpoint array is modified to allow more interconnection among the cells to provide communication between more cells. In the same line, Akers [23] introduced the "Rectangular Logic Array" where each cell in the cutpoint array receives an additional input from a non-immediate neighboring cell. This in practice makes the array to resemble a 
three-dimensional structure. Other than the above mentioned methods certained special purpose arrays have be reported [32] which utilize special features of their target structures and include Adder, Multiplier, threshold, sorting, coding, interconnection arrays, etc.

\subsection{Generic CA-type FPGA}

The Cellular FPGA's in the market inherit some of the characters of the above discussed cellular array. Such as identical cells with uniform interconnections between the cells, low fan-in/fan-out, and the fine granularity to implement the simple logic function or a storage element. The added feature of local and express busses for medium and long range communication makes them flexible from the synthesis point of view than the cellular arrays reviewed above. This makes them well suited for regular structured logic like datapath and cellular automata and for computer intensive applications [2] like convolution filtering, motion estimation of real-time video and real time image manipulation.

The generic cellular FPGA looks like the one shown in Fig 2.4. The CA-Type FPGA is regular array of locally connected programmable logic blocks. Each logic block is directly connected to a limited number of neighbors, usually four or eight, and to a small limited number of local and global (express) buses, usually four or eight, which are used for medium and long distance connections. Local and global buses run horizontally and vertically. In addition to logic and storage functions, cells can also be used as wires. These buses provide also link between the array and the I/O blocks. In homogeneous FPGAs, all logic blocks are identical except input and output ones. A set of logic functions which can be implemented in one logic block is defined by the block architecture. 


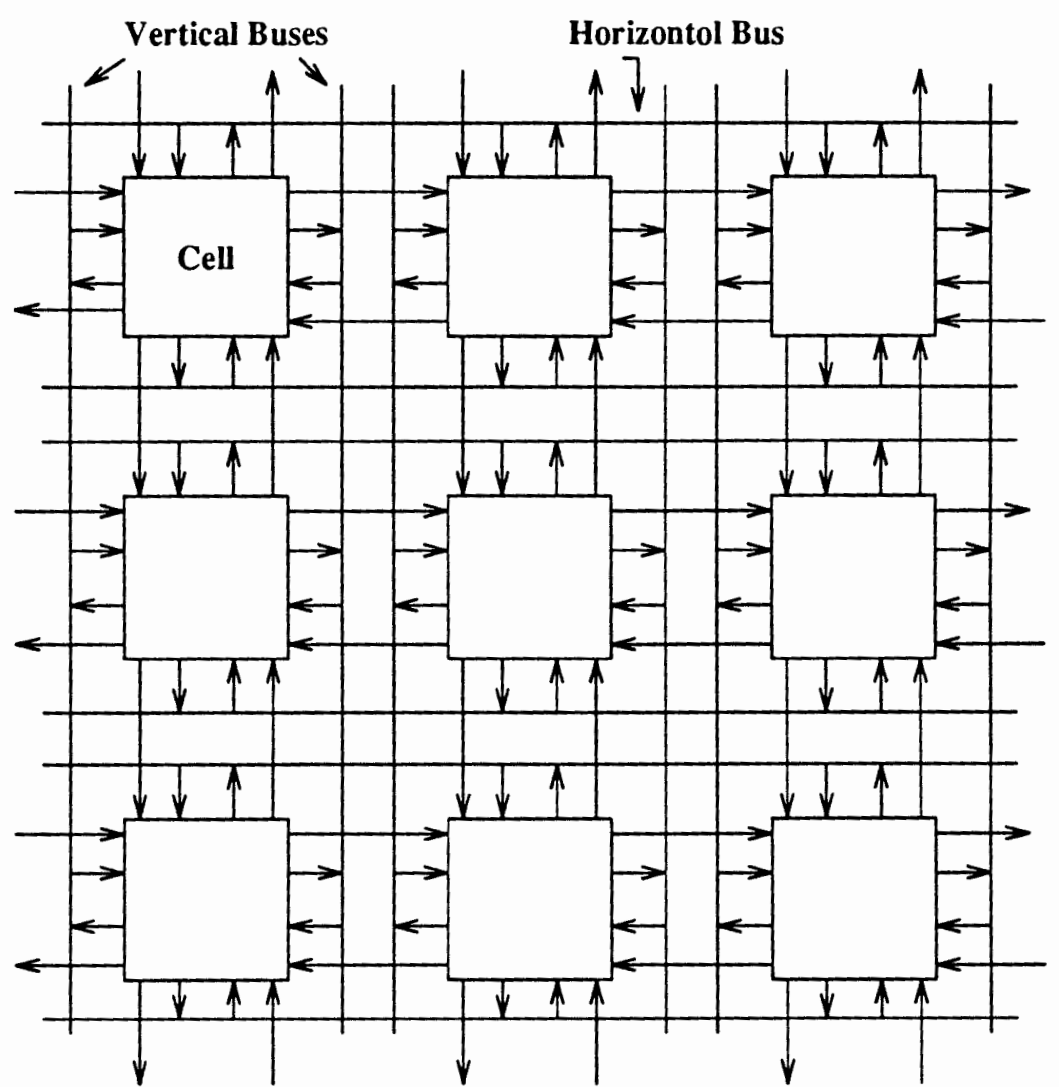

Figure 2.4 A Generic CA-type FPGA

Fig 2.5, Fig 2.6 and Fig 4.3 show the logic block from Algotronix [41], Motorola [42] and ATMEL [2] FPGA's, respectively. The Function Unit shown in Fig 2.5 implements the expected function, while the Mux's select the input signals to be applied to the function unit [41]. The logic cell shown in Fig 2.6 can be programmed as an AND gate. Alternately, each cell is capable of a secondary function such as D filp-flop, XOR and wired-OR gates. Logic synthesis and techonolgy mapping methods which can efficiently realize the functionality in logic blocks and optimally utilize routing resources should be developed. 


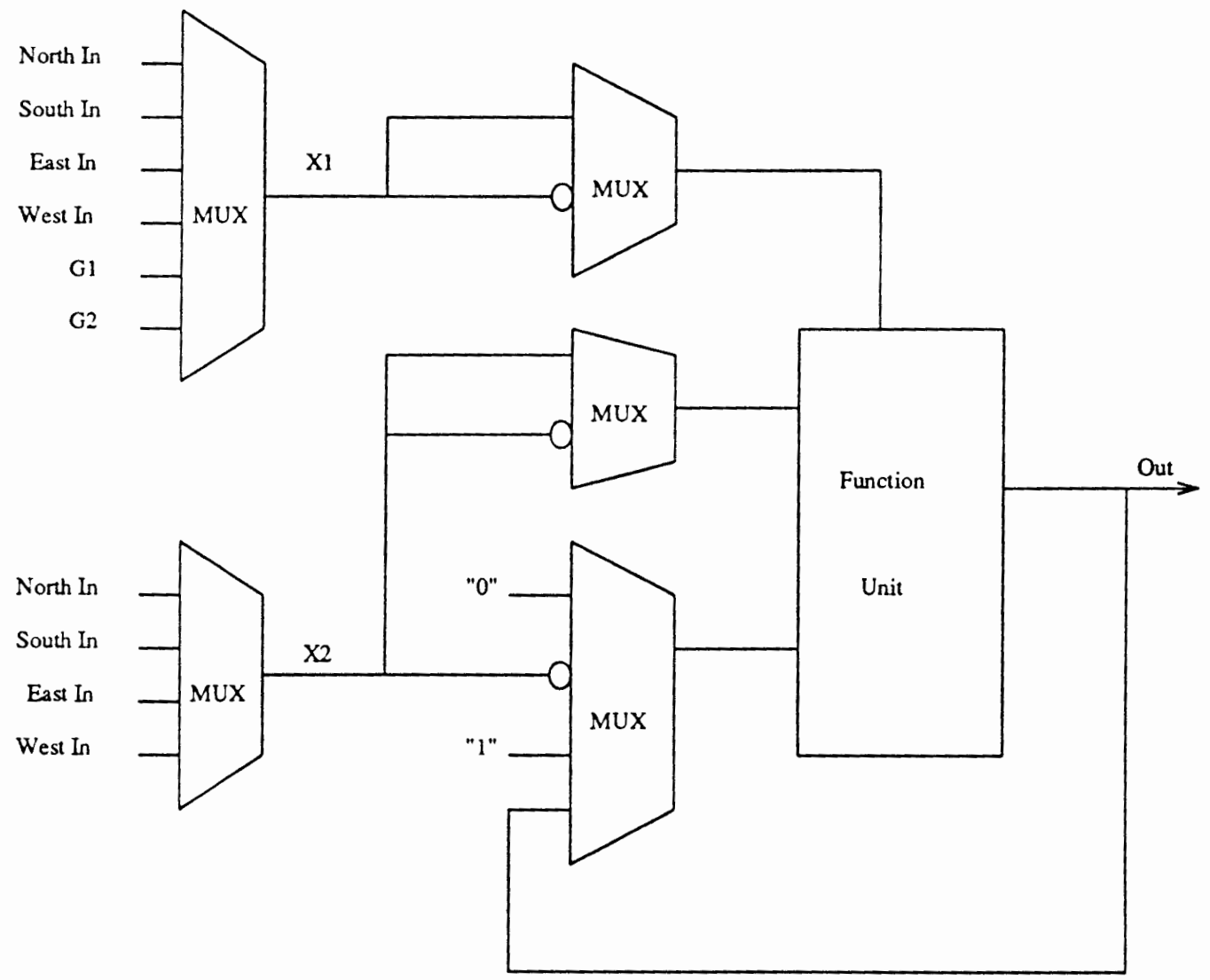

Figure 2.5 Logic Block of Algotronix FPGA

The industrial method for mapping functions into ATMEL FPGA is based on "Macro Generator Approach". The current research involved in logic and layout synthesis of these CA-type FPGAs are two-level AND/XOR realization, universal XOR (canonical forms of functions based on XOR) forms [16,17], Complex Matria Logic Array (CMLA) approach [12,11] and Simulated Evolution (SE) [12] method. The AND/XOR and the universal XOR form methods lead to fewer products than their AND/OR counterparts $[45,46]$, therefore requiring less layout area to implement the circuit. But to identify the minimal multi-level XOR canonical representation of a function with larger number variable the computation time is expensive. CMLA is based on the Maitra terms, and extension to the Maitra cascades [27,28,29]. A 


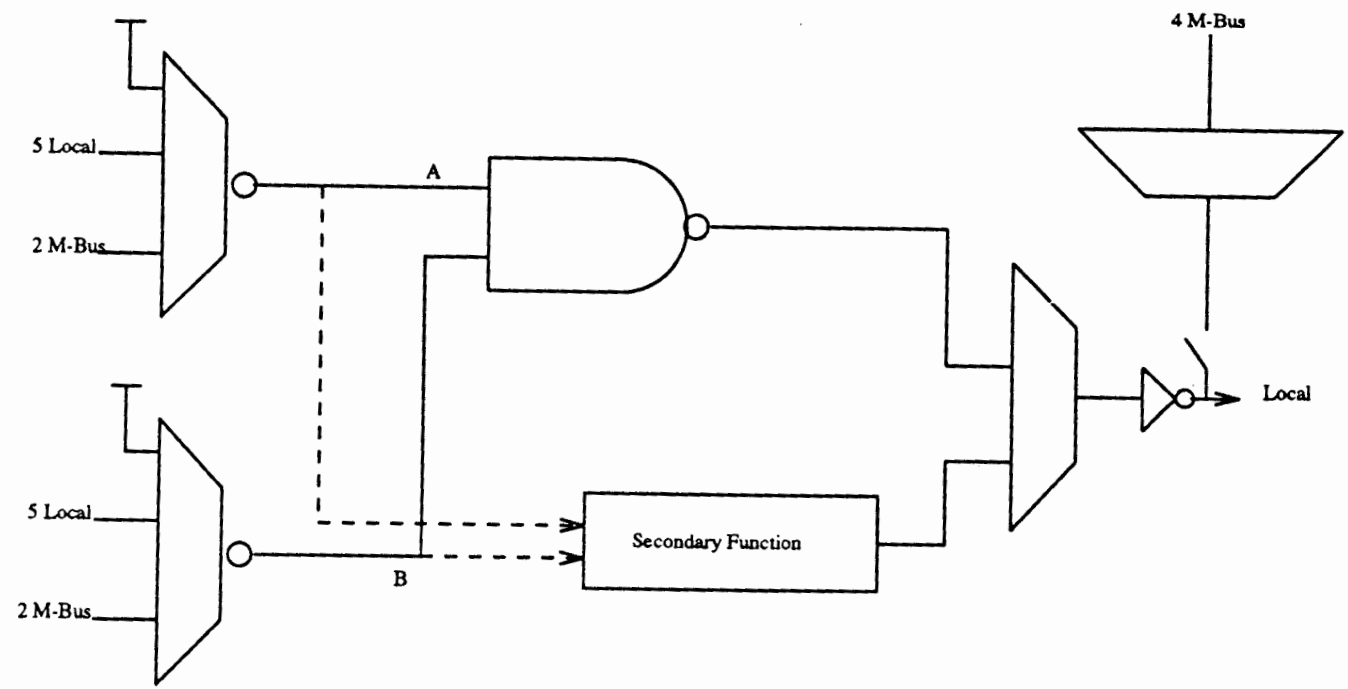

Figure 2.6 Logic Block of Motorola FPGA

complex term is a product term and is a sequence (row) of AND, OR and XOR operators with corresponding literals. In the CMLA, the input variables of the Boolean function are in vertical buses. The input plane consists of rows of logic cells representing the multi-level terms (Complex terms). The terms are then XORed/ORed together in the collecting (output) plane. A method based on linear sorting and SE performs mapping of a general DAG representing a Boolean function onto the chip considering the restrictions during the placement phase. Comparisons of these methods to our approach are made in Chapter VI. 


\section{CHAPTER III}

\section{LAYOUT-DRIVEN LOGIC SYNTHESIS}

A binary tree representation of the circuits is a very useful structure for mapping them to the CA-Type FPGAs, as the connections between logic blocks, represented as the vertices of the tree, are local and each node in the tree has only connections to its parent node and children nodes. In a binary tree the maximum number of connections for a node is three and therefore this configuration can be realized by using only adjacent logic blocks and local connections in the cellular array. The exponential growth of the number of nodes as a function of the tree level can, however, result in a very inefficient mapping. Fortunately, for most of the real functions, the shape of the tree is not getting as wide at the bottom as could be expected. The tree is expanding from the root for a few levels, then the width of the tree tends to stay constant, and decreases towards the leaves of the tree. Therefore, by developing a good restructuring method a binary tree can be easily mapped to the cellular architecture without wasting too many logic blocks for routing.

Logic synthesis methods based on EXOR gates are very attractive because they lead to functions being represent with a smaller number of gates (which means a smaller number of logic blocks needed to implement the circuit on the FPGA) and also because most of the FPGAs include EXOR gate in their logic blocks. For a long time, EXOR gate has been considered not useful for circuit implementation because its realization in silicon especially for large fan-in or fan-out was slow compared to other simple gates. However, with introduction of FPGAs, the delay of an EXOR gate became 
similar to the delay of other gates. For some types of FPGAs, like for example LUTbased Xilinx series, the delay of the logic bock depend on the number of input variables not on the functions realized by that block. In CA-type FPGAs which are based on small granularity of their logic blocks and localized connections, the fan-in and fan-out of the EXOR gate are low. Recently, EXOR gates have been more often used for the implementation of Boolean function due to easier testability [5]. It has been already shown that AND/EXOR representation of linear and nearly linear functions costs less (in a number of gates) than the inclusive (AND/OR) representation [5].

One of the most fundamental concepts for the decomposition of a logic function is the Shannon expansion. The Shannon expansion can always be applied to a logic function in contrast to other types of Boolean decompositions like the Ashenhurt [19] or the Curits [20] decomposition, which can be applied only to certain classes of functions. By applying certain rules to the Shannon expansion we can generate the Davio I and Davio II expansions as shown below. The well-known Shannon expansion is given by $[21,22]$

$$
\begin{gathered}
f\left(x_{1}, \ldots x_{i}, \ldots x_{n}\right)= \\
x_{i}, f\left(x_{1, \ldots}, x_{i}=1, \ldots x_{n}\right)+\overline{x_{i}} . f\left(x_{1}, \ldots x_{i}=0, \ldots x_{n}\right)
\end{gathered}
$$

By applying the rule

$$
\bar{a}=1 \oplus a
$$

We get the Davio I expansion:

$$
\begin{gathered}
f\left(x_{1}, \ldots x_{i} \ldots x_{n}\right)= \\
f\left(x_{1}, \ldots x_{i}=0, \ldots x_{n}\right) \oplus x_{i} \cdot\left[f\left(x_{1, \ldots}, x_{i}=0, \ldots x_{n}\right) \oplus\left(x_{1}, \ldots x_{i}=1, \ldots x_{n}\right)\right]
\end{gathered}
$$

Davio II expansion is derived applying the rule

$$
\begin{aligned}
& a=1 \oplus \bar{a} \\
& f\left(x_{1}, \ldots, x_{1}, x_{n}\right)= \\
& f\left(x_{1}, \ldots x_{i}=1, \ldots x_{n}\right) \oplus \overline{x_{i}} \cdot\left[f\left(x_{1, \ldots} x_{i}=0, \ldots x_{n}\right) \oplus\left(x_{1,}, x_{i}=1, \ldots x_{n}\right)\right]
\end{aligned}
$$


In short form we can represent the above expansions as:

$$
\begin{aligned}
& f=x_{i} f_{x_{i}}+\overline{x_{i}} f_{\overline{x_{i}}} \\
& f=f_{x_{i}} \oplus \overline{x_{i}} f_{x_{i}} \oplus f_{\bar{x}_{i}}=f_{x_{i}} \oplus \overline{x_{i} g} \\
& f=f_{\overline{x_{i}}} \oplus \overline{x_{i}} f_{x_{i}} \oplus f_{\overline{x_{i}}}=f_{x_{i}} \oplus \overline{x_{i} g}
\end{aligned}
$$

Where

$$
\begin{gathered}
f_{x_{i}}=\text { Cof actor w.r.t } x_{i} \\
f_{\bar{x}_{i}}=\text { Cofactor w.r.t } \overline{x_{i}} \\
\text { Boolean Difference } g=f_{x_{i}} \oplus f_{\bar{x}_{i}}
\end{gathered}
$$

The decompositions represented by equations (5) and (6) are called Davio I and Davio II, respectively. The circuit realization of equation (4) is given by a multiplexer gate while Equations (5) and (6) describe and AND-EXOR gate structure, as shown in Fig 3.1. Since we have chosen to use ATMEL 6000 series FPGA as our target architecture, and AND-EXOR combination can be realized in one logic cell of that architecture, we have selected input format accordingly. TRM package details will be explained in detail in the later chapters.

Any combination of Shannon and Davio I and II expansions can be used to produce decision diagrams [1,14]. A Binary Decision Diagram (BDD) is a Directed Acyclic Graph (DAG) with a single root node. The terminal (leaf) nodes represent the values 0 and 1, while non-terminal nodes represent Boolean functions. The function associated with the root node specifies the function represented by the entire BDD. Each non-terminal node has an associated variable and two outgoing edges. The function represented by the non-terminal node is specified by its cofactors with respect to its associated expansion variable. 
a. Circuit Realization of Equation (4):

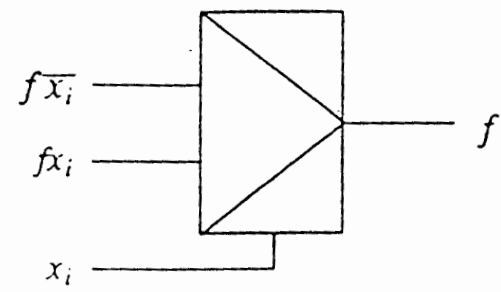

b. Circuit Realization of Equation (5):

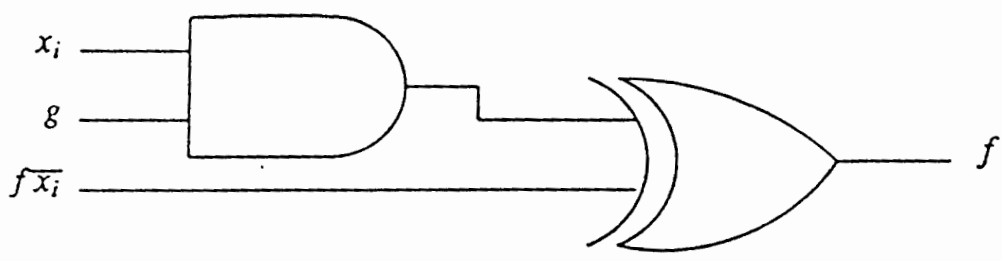

c. Circuit Realization of Equation (6):

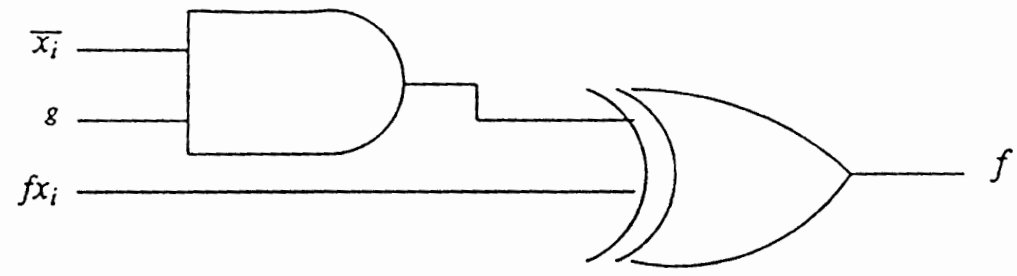

Figure 3.1 Circuit Realization of Shannon and Davio I and II Expansions

By applying only Davio I expansion to a given Boolean function, a Functional Decision Diagram (FDD), which is a binary tree with nodes representing AND and EXOR gates is created. This FDD, with only positive decomposition variable associated with each node of the binary tree is called Reed-Muller Tree (RMT) [1]. This tree is called Reed-Muller tree because once it is flattened, it represents a Reed-Muller canonical form. If the order of variables used for decomposition is the same in all branches of the tree then it is called Non-Permuted Reed-Muller-Tree (RMT). If the orders are not the same it is called Permuted-Reed-Muller-Tree (PRMT).

By applying different combinations of Shannon and Davio I and II expansions 
different decision diagrams can be obtained.

Generalized Reed-Muller Tree (GRMT): The expansion tree in which each of the variables appears only either in positive polarity or negative polarity. This is obtained by applying Davio I and II repeatedly. This results in a tree with AND-EXOR gate structure.

Pseudo-Kronecker Reed-Muller Tree (KRMT): The expansion tree in which all the variables appear either in positive polarity, negative or both polarities, but having a single fixed order of expansion variables in the tree levels. This is obtained by applying Shannon, Davio I and II repeatedly. This results in a tree with combination of AND, EXOR gates and Multiplexers.

All the above mentioned Decision Diagrams can be used for ATMEL 6000, by introducing an appropriate technology mapping (Grouping) algorithm to efficiently map the Tree onto the FPGA. We have chosen PRMT as the input to our algorithm as the technology mapping [4] was ready for the PRMT, at the start of this work.

PRMT of a given Boolean function, which is generated by the program REMIT [1], is used as the input to our algorithm. PRM Tree structure is very well suited for ATMEL FPGAs as it preserves the local connectivity between the cells and what is even more important matches the AND-EXOR configuration of the ATMEL logic block to its AND-EXOR structure.

\section{Example 1}

The Boolean function in the SOP (Sum-of-product) representation, which is used as the example to all steps of our method is given. The example is the benchmark function " $5 \times 1$ " from MCNC.

$\mathrm{f}=\bar{a}$ bcdef $\bar{g}+\bar{a}$ efg + aef 
The pla format of the function is as follows.

.i 7

.01

.p 3

01111101

$0---1111$

$1---11-1$

.e

Where

.i specifies the number of inputs (variables).

.o specifies the number of outputs.

.p specifies the number of product terms (cubes)

0 the variable is in negative polarity

1 the variable is in positive polarity

- the variable is not in the product term (don't care)

Using the program REMIT[1], the PRMT for the Boolean function from example 1 is generated, as shown in Fig. 3.2.

The PRMT is used as the input to our algorithm. On Fig. 3.2 the root of the tree represents the output of the function. The logic gates are represented with generic logic gate symbols and the primary (expansion) variables $(a, b, c . .$.$) which are associated$ with each node are also shown. 


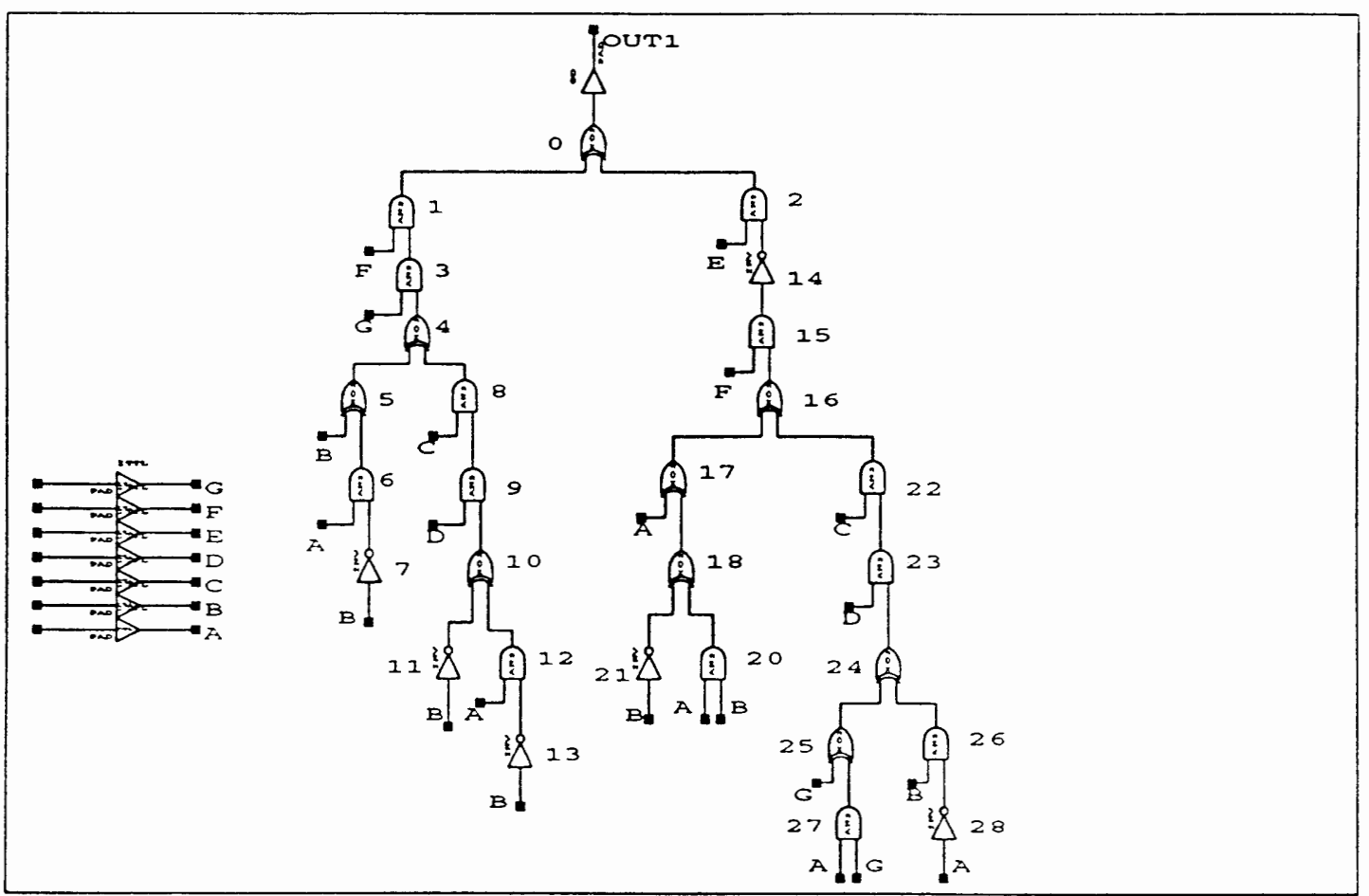

Figure 3.2 PRM Tree of the Boolean Function from Example 1 


\section{CHAPTER IV}

\section{ARCHITECTURE OF ATMEL 6000 FPGA}

The logic synthesis method discussed in Chapter III combined with the "Tree Restructing Method", which will be discussed in the later Chapters, will lead to a complete comprehensive package which can take a function specified in pla format and generate the final layout in any cellular architecture. In this work, we have included technology mapping stage specific to ATMEL 6000 chip. Because, the technology mapping targets ATMEL 6000 chip, the general features of the architecture are reviewed and the main restrictions that lead to the presented algorithms are pointed out in this chapter.

\subsection{The Symmetrical Array}

At the heart of the ATMEL 6000 architecture is a symmetrical array of identical cells which is shown in Fig 4.1. Except for "repeaters" spaced every eight cells, the array is continuous and completely uninterrupted from one edge to the other. In addition to logic and storage functions, cells can also be used as wires. Buses support fast, efficient communication over medium and long distances.

\subsection{The Bussing Network}

There are two kinds of buses: local and express as shown in Fig 4.2. Express buses are not directly connected to the cells, they are used for global connections via the local buses. Local buses are the link between the array of cells and the global 


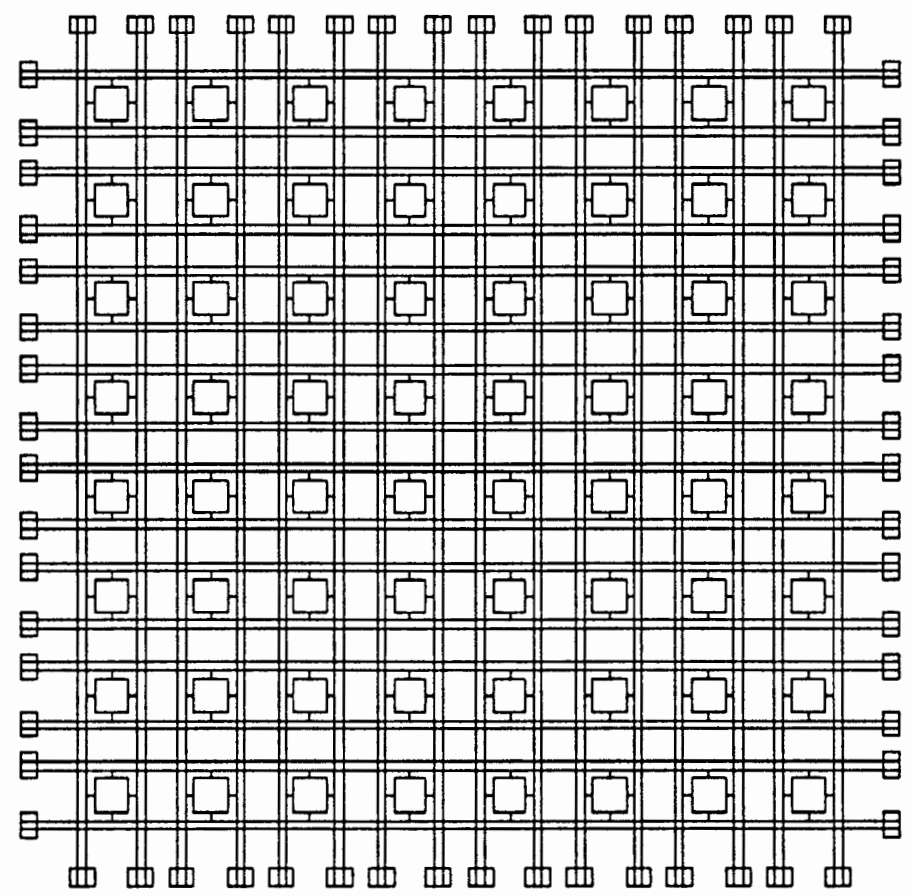

Figure 4.1 Symetric Array of the ATMEL 6000 Chip

bussing network. There are two vertical local buses for every column of cells, and two horizontal local buses for every row of cells. Every cell in the array has a read/write access to two vertical and two horizontal buses. Each cell, in addition, provides the ability to make a 90 degree turn between either of the two vertical buses and either of the two horizontal buses. Express busses are the fastest way to cover long, straight-line distances within the array. Each express bus is paired with a local bus, so there are two express buses for each column and row of cells. Connective units called repeaters, spaced every eight cells, divide each bus, both local and express, into segments spanning eight cells. Each repeater is associated with a local/express pair. 


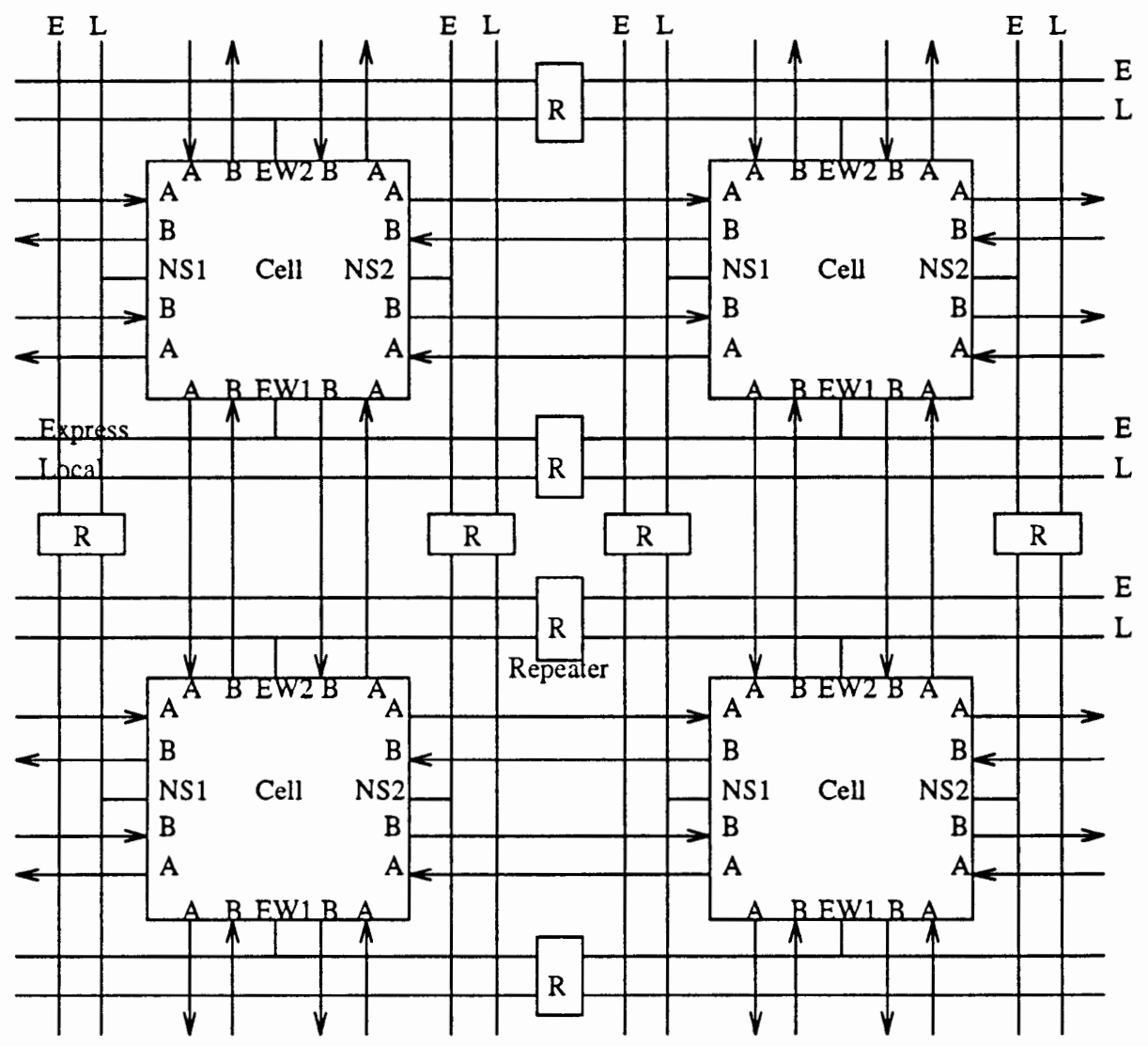

Figure 4.2 Bussing Network

\subsection{Cells States}

There is one base cell as shown in Fig 4.3 from which thirty five logical functions can be implemented. Out of these functions twenty are purely combinatorial cell states providing all primitive logic functions like NOR, NAND, AND, OR, 2-input multiplexer, and some combinations of the primitive gates as shown in Fig 4.4. 


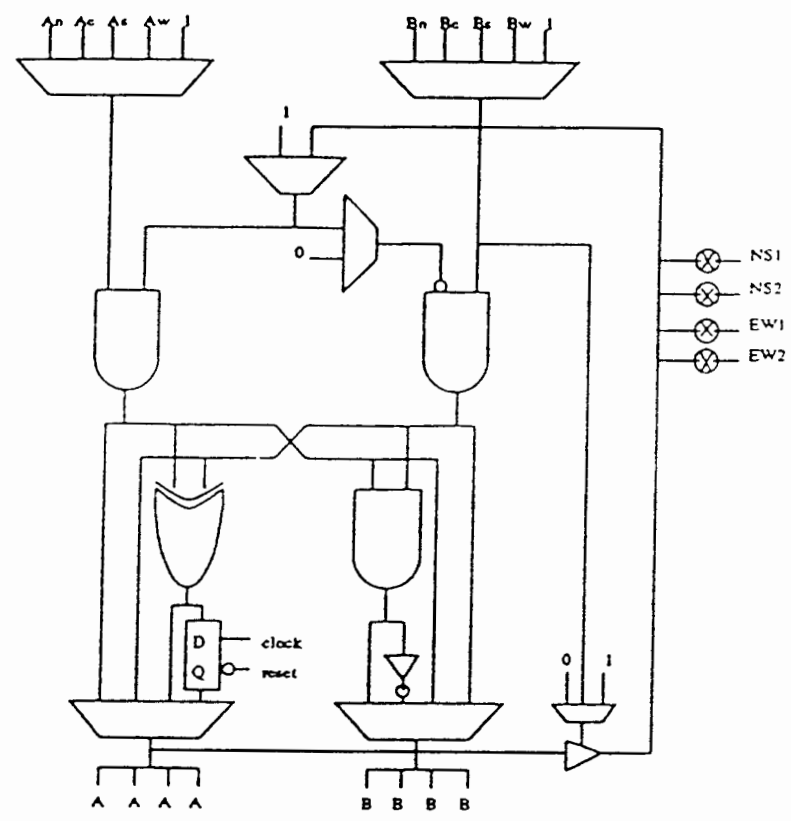

Figure 4.3 Basic Cell
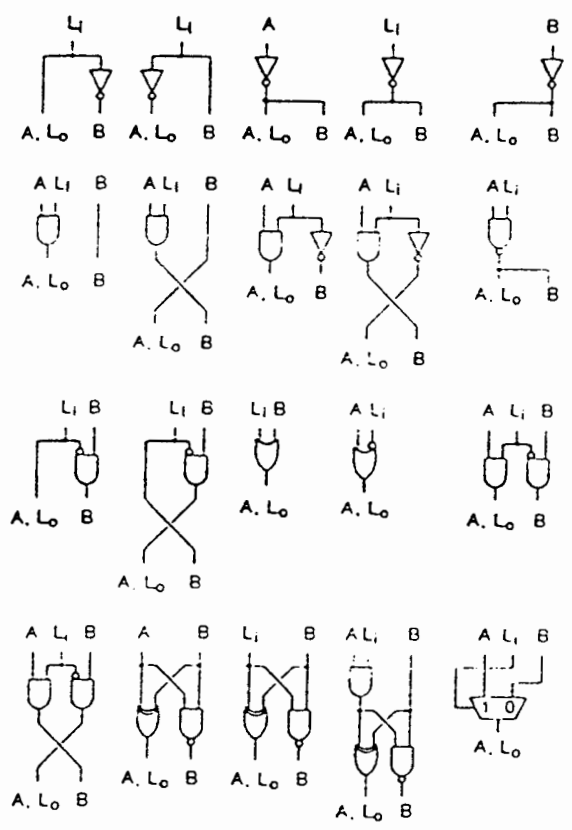

Figure 4.4 Combinatorial States 
AND/EXOR realization is of special interest to us. The logic synthesis method REMIT discussed in Chapter III, decomposes a Boolean function to an AND/EXOR tree. The AND/EXOR realization in a logic cell of ATMEL 6000 is shown in Fig 4.5.

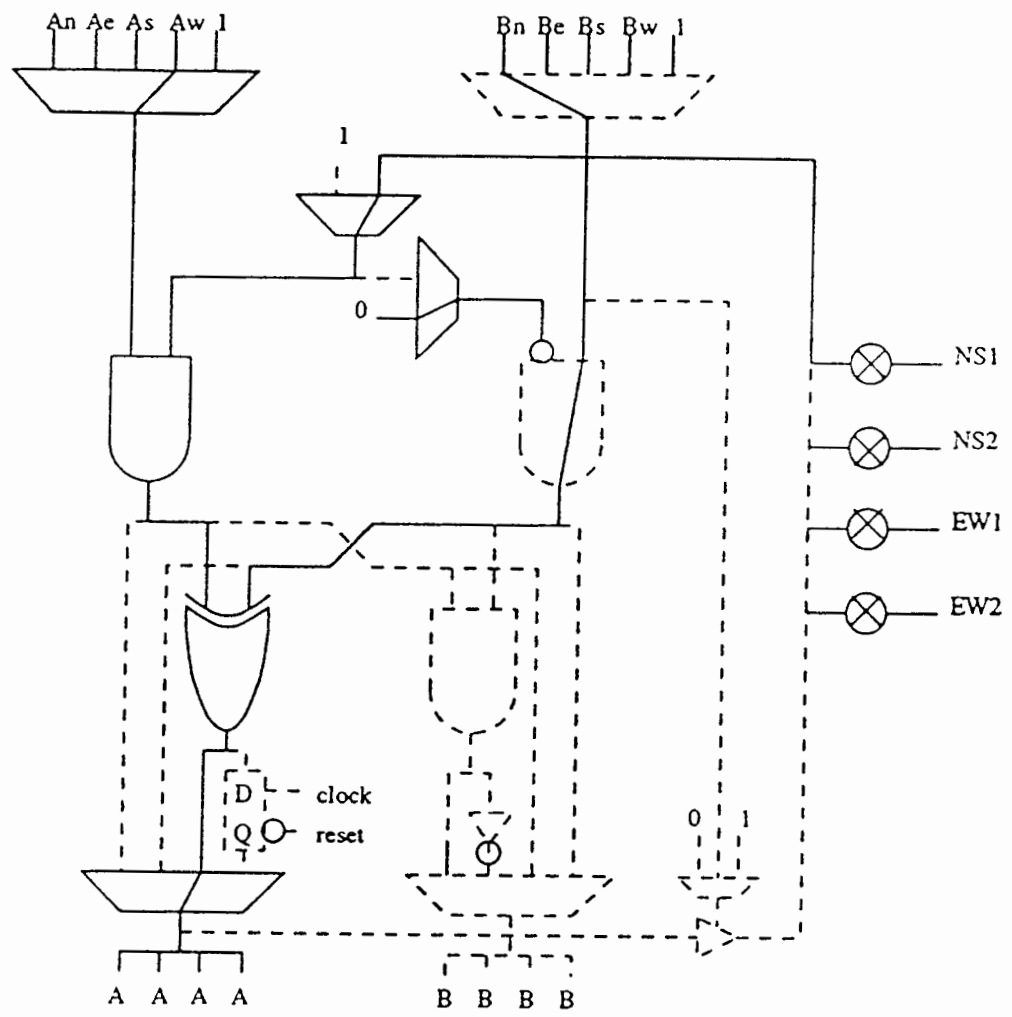

Figure 4.5 AND-EXOR Realization

Also supported are six register states and four constant states shown in Fig 4.6.

In addition to the four local bus connections, a cell receives eight inputs and provides two outputs to its North, South, East, and West neighbors. These ten inputs and outputs are divided into two classes: $A$ and $B$. There is an $A$ input and a $B$ input for each neighboring cell and a single A output and single B output driving all four neighbors. For outside connections, an $\mathrm{A}$ output is always connected to an $\mathrm{A}$ input and a $\mathrm{B}$ output to a $B$ input. 

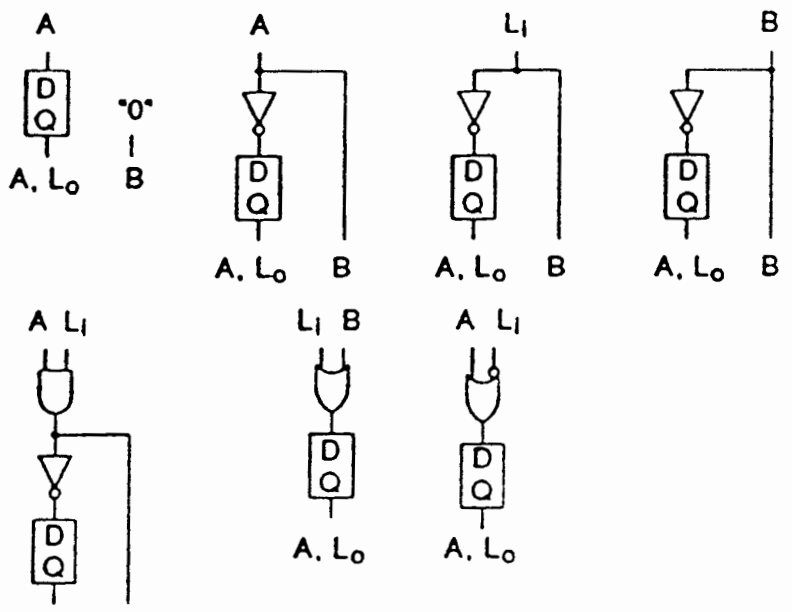

A. Lo B

A. LO B

A. Lo B

A. Lo 8

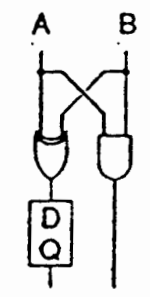

$4 B$

A $L_{1}$

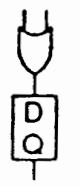

$\theta$

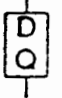

A. $L_{0}$

A. Lo

A. Lo $B$

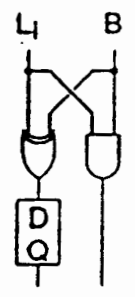

A. L L $^{8}$
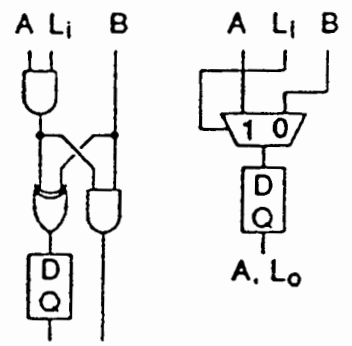

A. $L_{0} B$

A. L。

B

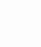




\subsection{Architecture Restrictions}

Each node can have only one input from the local bus and at most two inputs from the neighbors. All possible input configurations can be described as follows and are shown in Fig 4.7.

1. 2 inputs from adjacent cells (one must be to ' $A$ ' input and the other to ' $B$ ' input)

+1 input from local bus.

2. 2 inputs from adjacent cells (one must be to ' $A$ ' input and the other to ' $B$ ' input)

3. 1 input from adjacent cell +1 input from local bus.

4. 1 input from local bus.

5. 1 input from adjacent cell.
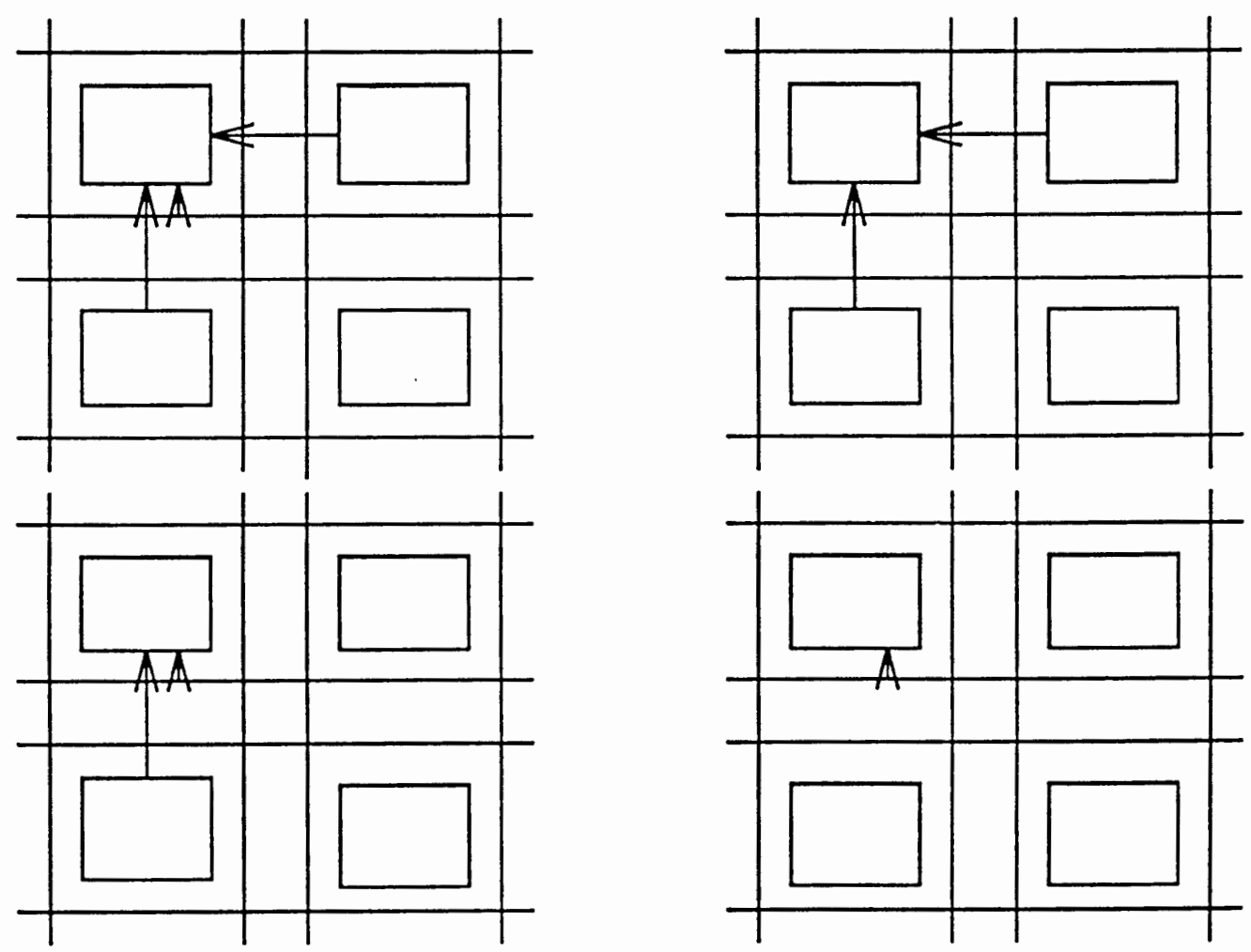

Figure 4.7 Four Possible Input Configurations to a Cell 


\section{CHAPTER V}

\section{TREE RESTRUCTURING APPROACH}

In this Chapter the general tree restructing approach based on the Squashed Binary Tree (SBT) developed here will be discussed, and the problem formulation is presented.

\subsection{TREE RESTRUCTURING}

In our approach, we generate the Boolean network in the AND/EXOR tree form which can easily be realized in the target ATMEL 6000 architecture. The main feature of architecture, local connectivity is also preserved in this AND/EXOR tree form. Furthermore, the tree restructuring method is used to generate the planar embedding of that Boolean network, such that no routing constraints are violated. The routing restrictions which are used in our algorithm are defined by the generic CA-type FPGA architecture and by specific limitation of the ATMEL chip. We assume that each gate has only "one output" in a generic CA-type FPGA. The inputs are limited to a maximum of "3", dictated by the limitations of ATMEL architecture which were stated in Chapter IV.4. If the tree is mapped directly onto the cellular array without restructuring, many cells and local buses are wasted for routing. This impacts directly the throughput of the chip, as the utilization factor L/R [a number of cells used for logic (L)/a number of cells used for routing $(R)$ ] will be low if more cells are used for routing than for logic in a given design. By using the stated constraints, the tree is restructured in such a way that when it is mapped to a flat surface the shape of the embedded tree is close to a 
rectangle and routing resources are used efficiently.

To achieve this goal, first the squashed binary tree algorithm [3] is used to reshape the representation of the binary tree. The squashed binary tree is formed by projecting nodes of the binary tree onto its leaves. Starting from the root, each node is projected onto its left-most descendant. Whenever a node has two child nodes, this process is repeated starting with the child node which was not projected earlier. Fig 5.1 (b) represents squashed binary tree for the binary tree shown in Fig 5.1(a).

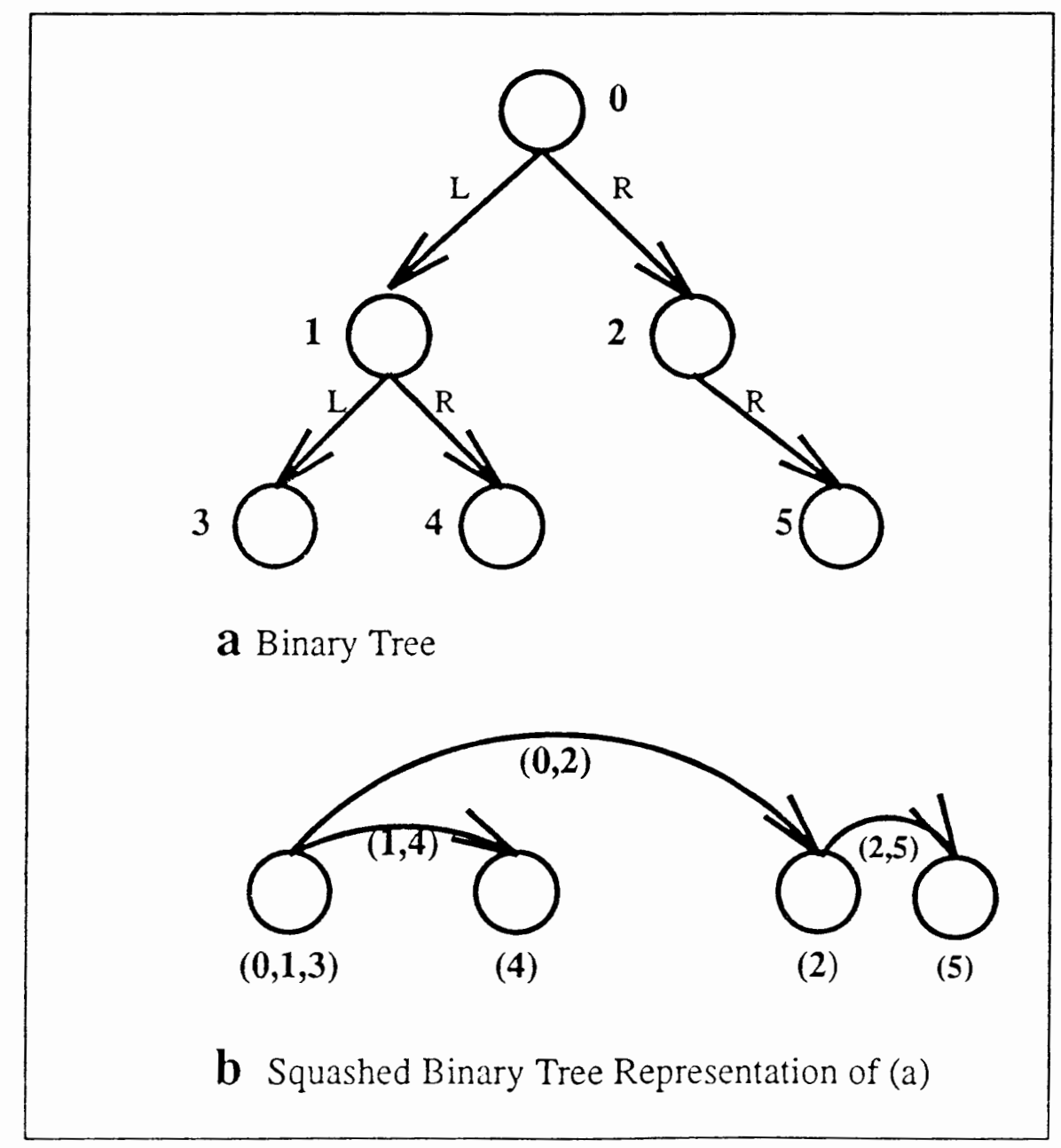

Figure 5.1 Binary Tree to Squashed Binary Tree Transformation 


\subsection{MAPPING PROBLEM FORMULATION}

PRMT which represents a given Boolean function is modeled as a rooted acyclic graph $T=(V, E)$ which consists of the ordered set of vertices $V$, and the set of directed edges $\mathrm{E}$ defined as follows:

* $V=\left\{v_{j} \mid v_{j}\right.$ represents nodes of the PRM Tree which can be realized in one logic block of a given architecture or a wire cell \}

* $E=\left\{e_{j} l_{j}\right.$ is an edge from $v_{j}$ to $v_{j+1}$, which represents a functional relation between these two nodes\}. Direction of the edge represents the direction of signal flow in the actual design. The vertices of the graph $\mathrm{T}$ are labeled with the primary signals (expansion variables) entering the nodes represented by the vertices. The fan-out of each $v_{j}$ is equal to 1 and the fan-in of each $v_{j}$ is not greater than 2 .

The physical resources of the CA-type FPGA are represented as the undirected graph $G_{p}\left(V_{p}, E_{p}\right)$ with the ordered set of vertices, $V_{p}$, and a set of edges $E_{p}$ defined as $* V_{p}=\left\{v_{p} \mid v_{p}\right.$ represents a logic cell of the CA chip. $\}$

$* E_{p}=\left\{e_{p} \mid e_{p}\right.$ represents the programmable connections between the adjacent cells $\}$

The vertices are numbered according to their positions in the column-row matrix of the CA-type chip.

Mapping Problem Formulation: Given the undirected physical graph $G_{p}\left(V_{p}, E_{p}\right)$ and the network graph $\mathrm{T}(\mathrm{V}, \mathrm{E})$ representing a design, find a mapping of the graph $T$ to the physical graph $G_{p}$ that satisfies the routing constraints of the given architecture and that minimizes the rectangular envelope (area) covered by the design and the number of logic blocks used for routing.

The main advantage of the described here approach is that we have eliminated the traditional routing phase to realize digital logic. Once SBT is created it can be directly mapped to the CA-type array, and only the necessary logic block assigned for routing 
need to be added.

The input to the SBT algorithm is the a $\mathrm{T}(\mathrm{V}, \mathrm{E})$ which represents the netlist in the form of the Permuted Reed-Muller Tree generated by the REMTT program. The primary (expansion) variables associated with the vertices of the PRMT tree will be distributed on the chip using local buses.

\subsection{TRM Flowgraph}

The overall flow graph of the Tree Restructuring Method (TRM) is shown Fig. 5.2. The input to the logic optimization phase is in pla format. The output of logic optimization phase is a binary tree. In this thesis the logic optimization program REMIT is used, to produce the PRMT representation of the given function. Next an optional technology mapping step is introduced to perform technology specific optimization. The tree is then restructured using SBT technique and finally the primary inputs are assigned to the local buses in the bus assignment phase. The sub-sections in Chapter VI give a description of the algorithms used in the TRM. Formal description of the steps in TRM package are as follows

STEP 1: Generate Squashed Binary Tree.

The nodes of the SBT are labelled as $\mathrm{v}_{\mathrm{b} 1}, \mathrm{v}_{\mathrm{b} 2} \ldots . ., \mathrm{v}_{\mathrm{bn}}$, where $\mathrm{n}$ is the number of leaf nodes in the original PRMT tree. Each node $v_{b i}$ is a set of vertices $\left(v_{1}, v_{2}, \ldots . . . v_{m}\right)$ of the PRMT tree, which were collapsed to that node. An edge exists between $v_{b i}, v_{b j}$ if there exists an edge $e_{k l}$ between $v_{k}$ and $v_{l}$, where $v_{k}$ is a vertex which was collapsed to node $\mathrm{v}_{\mathrm{bi}}$, and $\mathrm{v}_{1}$ is a vertex which was collapsed to node $\mathrm{v}_{\mathrm{bj}}$.

STEP 2: Perform mapping of the SBT to the target CA-type FPGA.

For all nodes $\mathrm{v}_{\mathrm{bi}}$ of the $\mathrm{SBT}$, place all vertices belonging to $\mathrm{v}_{\mathrm{i}}$ in the same column of the target cellular array. Node $\mathrm{v}_{\mathrm{bi}}$ will occupy as many rows as there are vertices belonging to that node. First place all vertices $v_{i}$ belonging to $v_{b 1}$ in column one. 


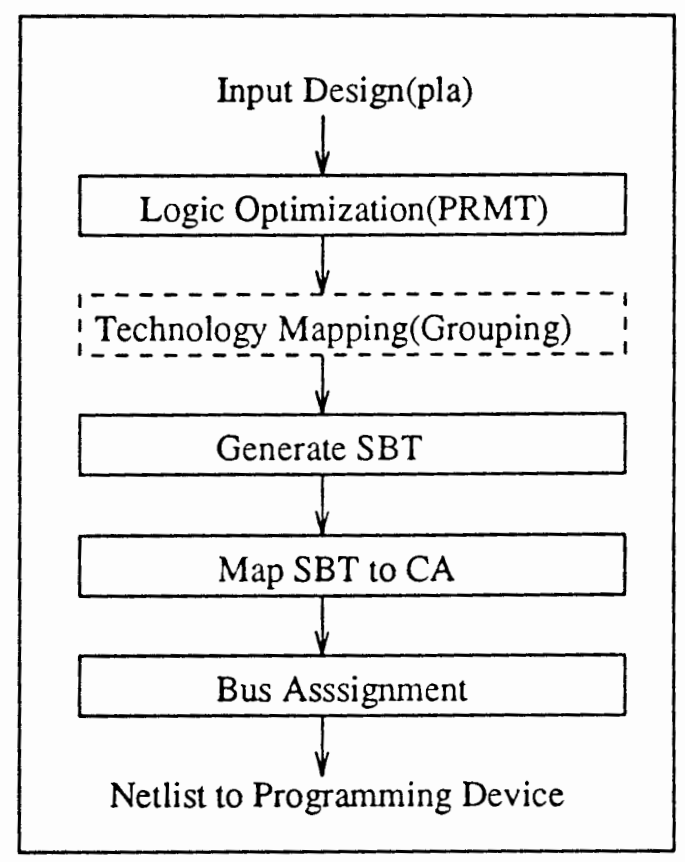

Figure 5.2 TRM Flowgraph

Then place vertices belonging to $v_{b 2}$ in column two staring with Row(j) defined by the edge between $v_{b 1}$ and $v_{b 2}$. The edge between $v_{1}$ and $v_{2}$ is defined by the edge between $v_{k} \in v_{b 1}$ and $v_{j} \in v_{b 2}$, where $j$ is the number of the row $v_{k} \in v_{b 1}$ is placed on the cellular array. This mapping is continued until all nodes are placed. Add additional routing cells if required.

STEP 3: Perform bus assignments.

The steps of the bus assignment algorithm are explained in Chapter VI.4

Based on the experiments we noticed that more compact shape with a smaller number of routing cells could be achieved, by relaxing the left-most-descendant constraint used to construct the SBT. We have introducted a new algorithm called Modified Squashed Binary Tree (MSBT) which gives better results both in the rectangular area and the number of routing cells used by the given design (Ref. Tab I) than the SBT implementation. The MSBT will be explained in detail in Chapter VI. 


\section{CHAPTER VI}

\section{DESCRIPTION OF ALGORITHMS}

In this Chapter we discuss the Tree Restructuring Method (TRM) package which includes Technology Mapping (Grouping), Modified Squashed Binary Tree (MSBT), and the Bus Assignment alogrithms. We discuss the extention of this method to multioutput functions. We compare TRM with other methods used to realize digital logic in CA-type FPGAs.

\subsection{TECHNOLOGY MAPPING}

In general, any decision diagram or binary tree can be used as input to our restructing algorithm, but to get better results a tree which can provide the best matching between structure of the tree and a given FPGA should be selected. PRM tree structure is very well suited for ATMEL FPGAs as it preserves the local connectivity between the cells and gives the flexibility to performing technology mapping for efficient utilization of available resources, i.e both the cells and buses.

The logic synthesis program REMIT produces a PRM tree with EXOR, AND and NOT gates. Though PRM tree preserves the local connectivity; it cannot be used directly as the input to the restructuring algorithm (which will be discussed later) as it violates certain architecture constraints of ATMEL 6000 series FPGAs. For example, in Fig 6.1 we see that from netlist of REMIT, output of one EXOR gate is directly connected to the input of another EXOR gate but in ATMEL 6000 this cannot be realized. 
The architecture constraint (type mismatch) states that Output type 'B' cannot be connected to Input type 'A'. The "switch cell" is introduced between the parent cell and one of its leaf cell as shown in Fig 6.2.

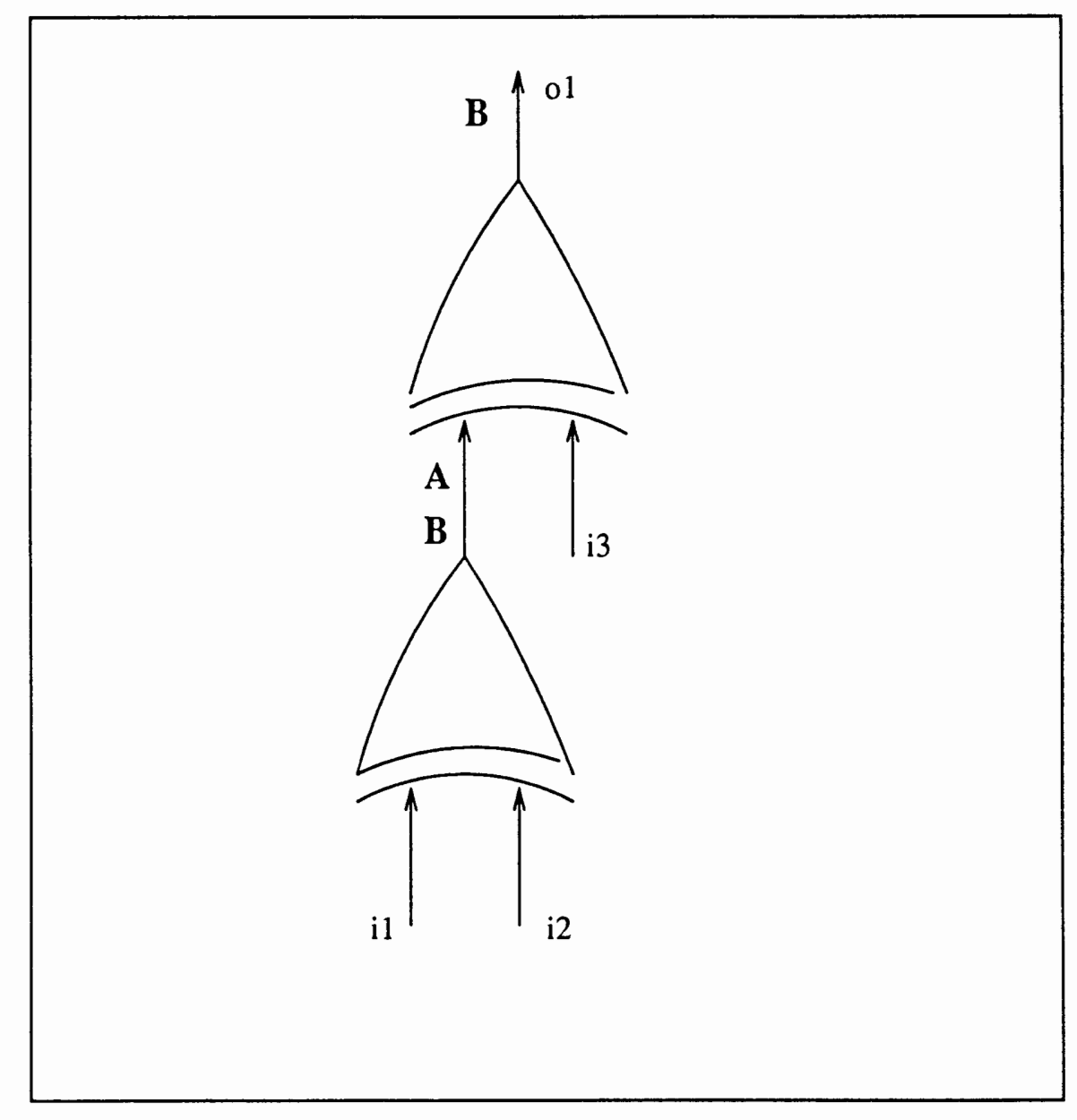

Figure 6.1 Illustration of Type I Mismatch

Similarly, a "Connection cell" is added when two signals assigned to local buses have to enter the same cell; because only one signal can be taken from the local bus as shown in Fig. 6.2. In this phase a grouping of cells is done to save area as well as improve performance. For example the tree shown in Fig 6.3 has EXOR at level 1 


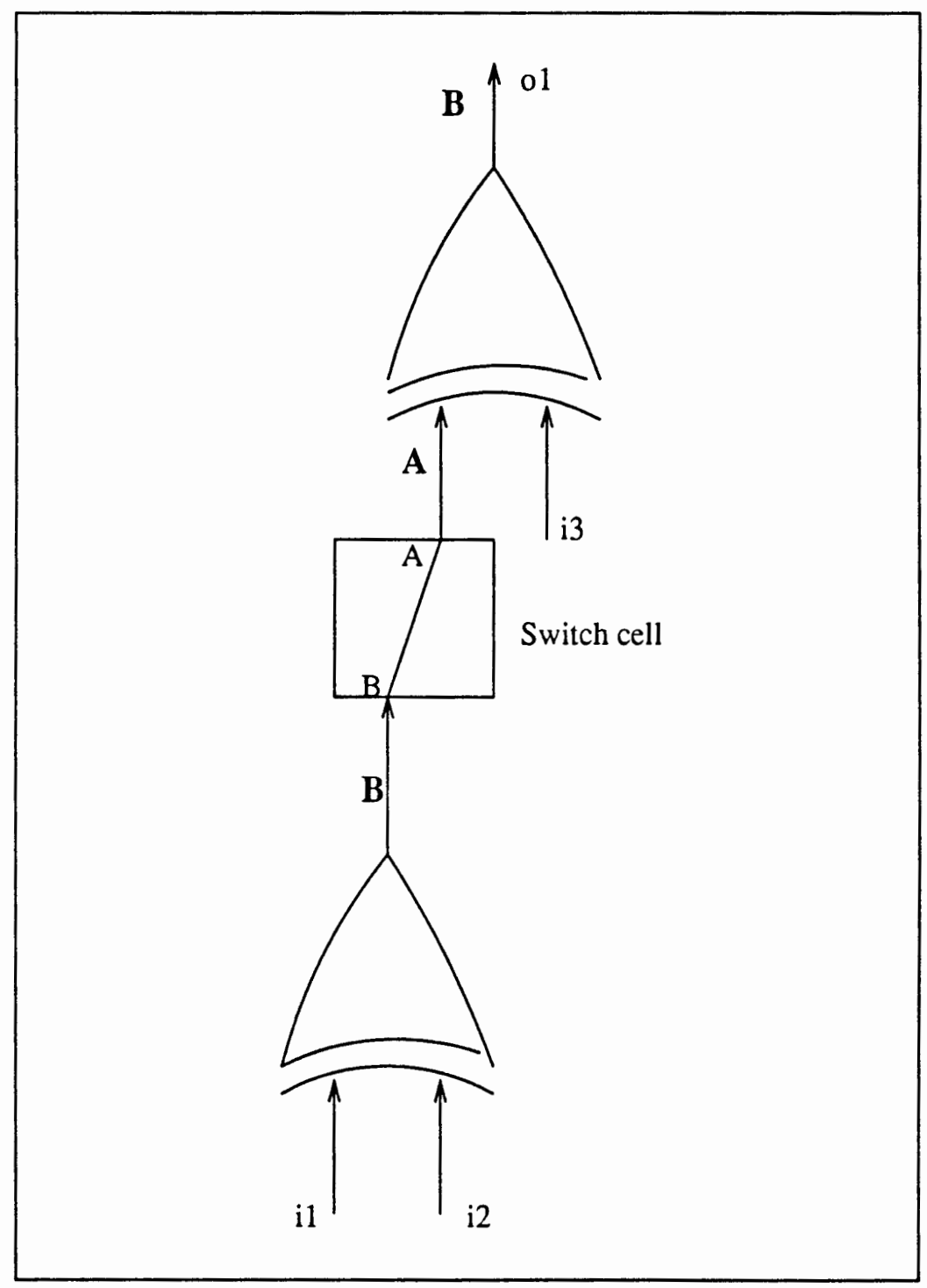

Figure 6.2 "Switch Cell" - Solution to Type I Mismatch

and AND at level 2 of the tree, but in ATMEL 6000 this can be realized in one cell as shown in Fig 6.4. The effect of such grouping is that we save one cell (area) and improve performance because the delay for each cell in FPGA's is almost the same and by reducing a number of cells in the path we reduce delay by one unit. 


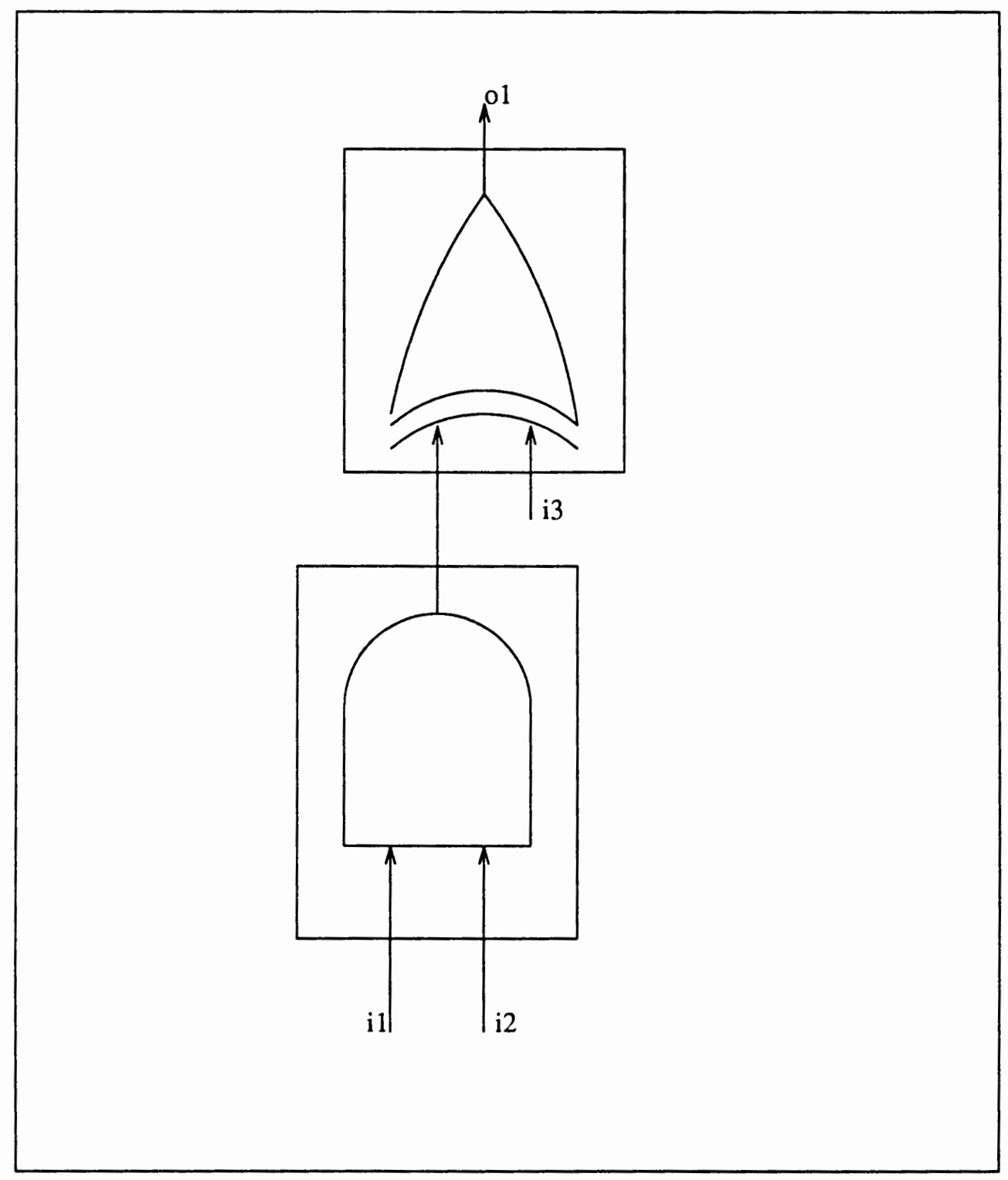

Figure 6.3 A Two Cell Realization of EXOR-AND Chain

The technology mapping is performed using a simple grouping algorithm [4] which analyzes the tree and groups successive AND and EXOR gates into one node and adds wire cells to overcome the architecture constraints.

In Fig 6.5 the grouped PRM tree for the example from Chapter III (Fig 3.2) is 


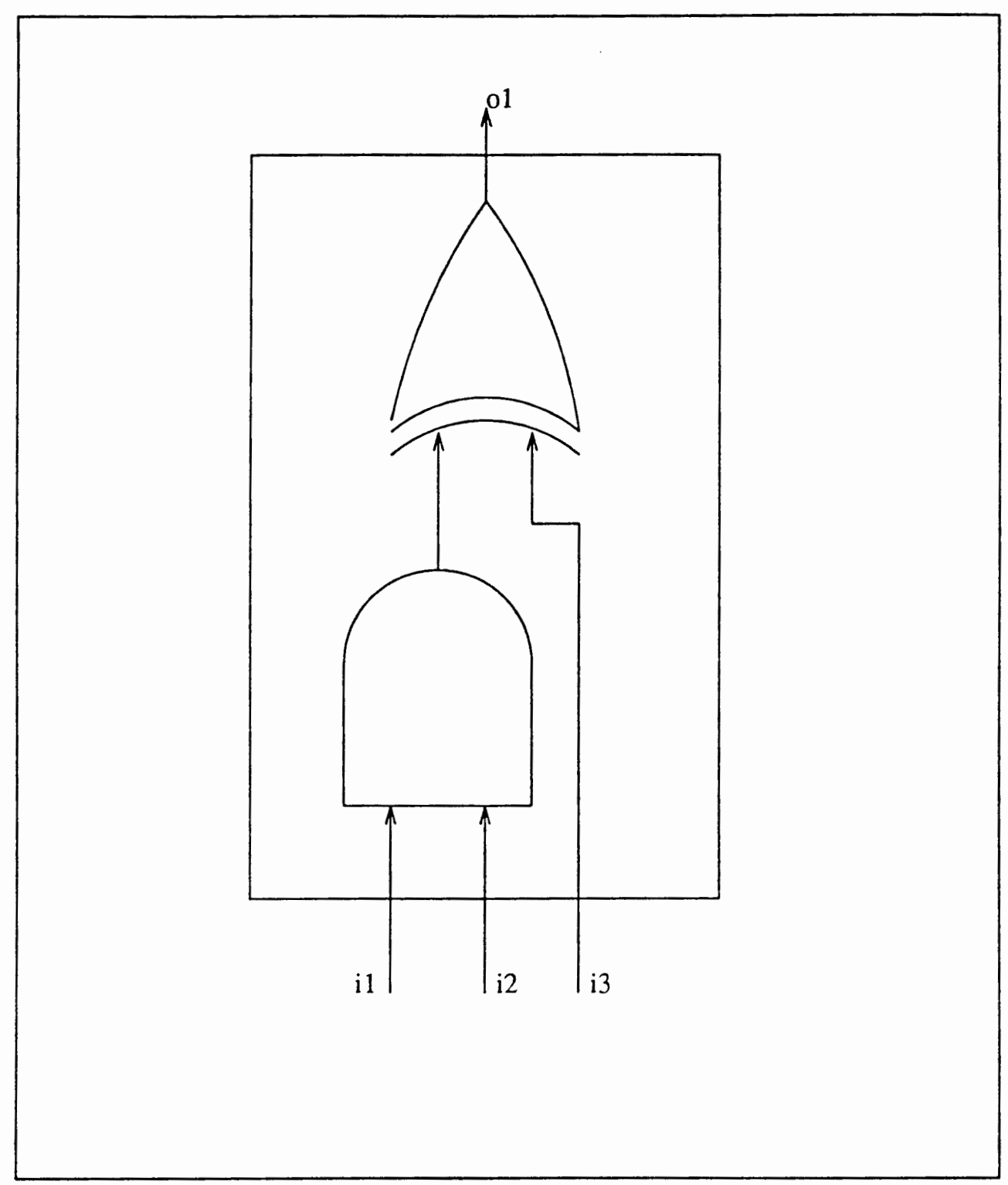

Figure 6.4 One Cell Realization of EXOR-AND Chain

shown. To simplify the representation of the circuit shown in Fig 6.5, the binary tree representation of that circuit is shown in Fig 6.6, where nodes represent gates and the connections between gates are represented as directed edges indicating the direction of signal flow. For easy comparison of the two representations, the gate numbers from Fig 6.5 are given as node numbers of the corresponding nodes in Fig 6.6. 


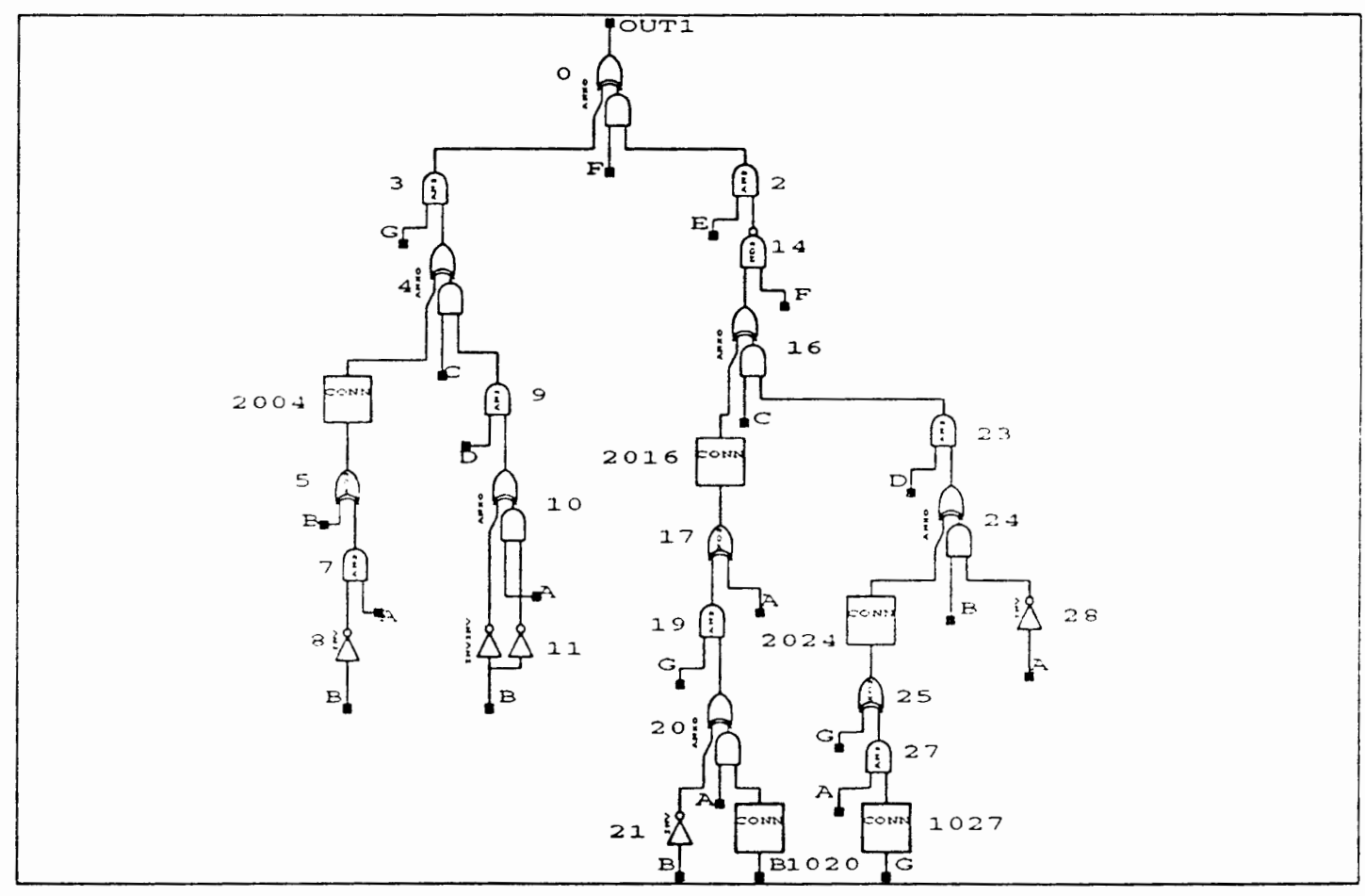

Figure 6.5 Grouped PRMT of the Example from Figure 3.2 


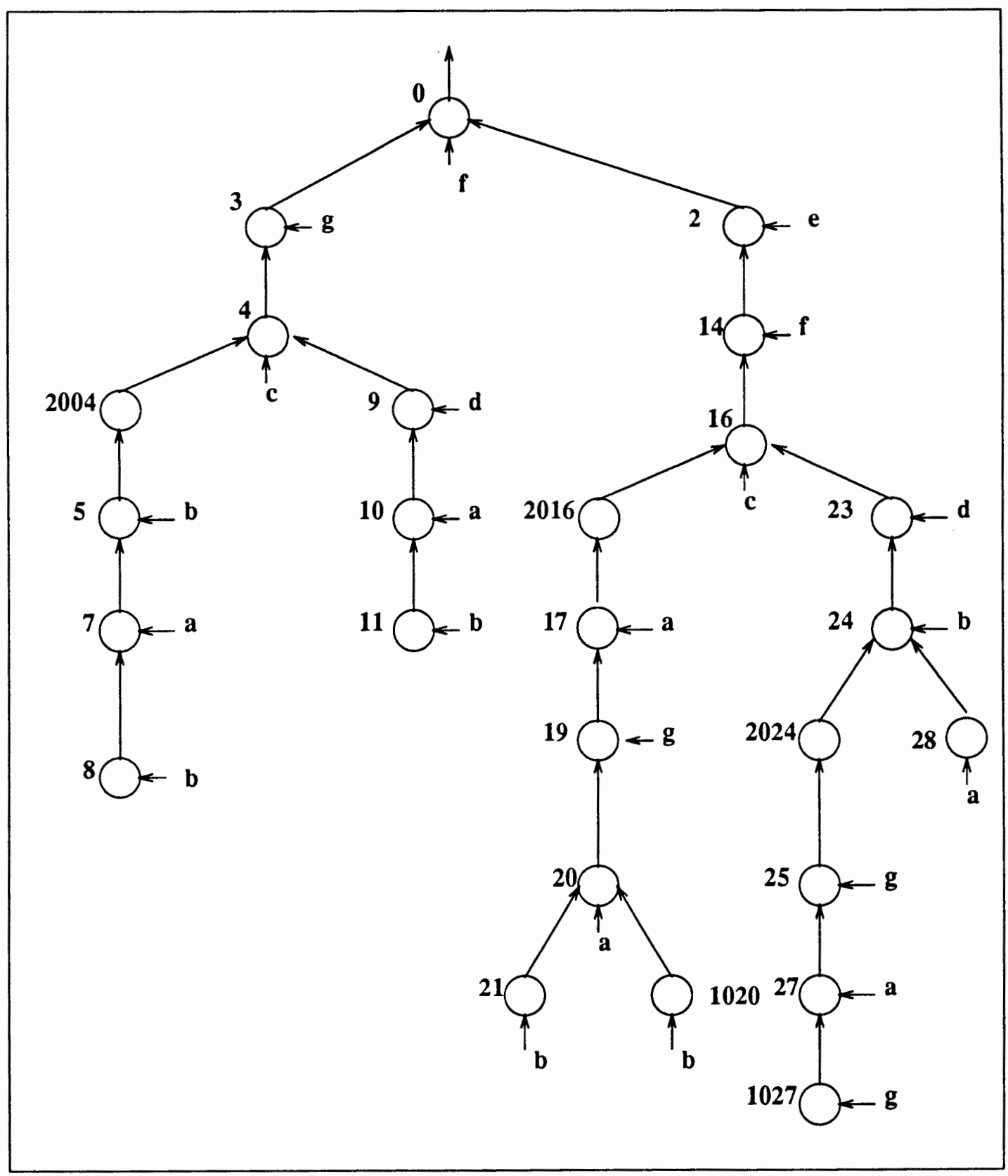

Figure 6.6 A Grouped PRMT as a Binary Tree 


\subsection{SQUASHED BINARY TREE}

The Squashed Binary Tree approach was chosen as it gives us a possibility to shape the tree into a rectangular form which closely represents the CA-type architecture. The rectangular shape is easy to map to the array while satisfying the design restrictions. Mapping a SBT to the CA-type architecture is just a straight-forward process as we place each node of the SBT into one column of the target array and make the neccessary connections.

The squashed binary tree is formed by projecting the binary tree onto its leaves. Starting from the root each node is projected onto its left-most descendant. The process will be repeated starting with the child nodes which were previously not projected, by traversing the tree in the bottom-up direction. In Fig 6.7 the squashed binary tree representation

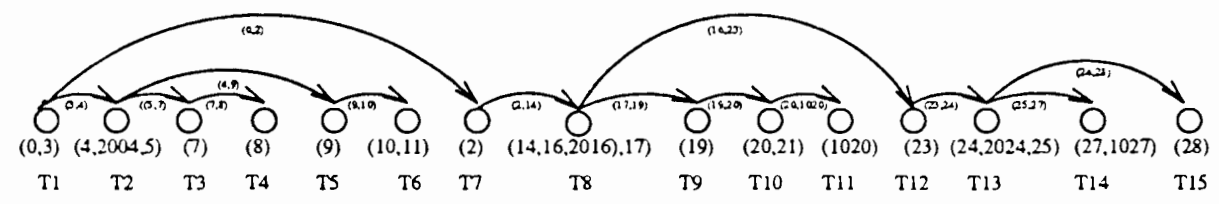

Figure 6.7 Squashed Binary Tree (SBT)

of the PRM tree from Fig 6.6 is shown. T1 ... T15 are the nodes of the SBT. Fig 6.8 shows the mapping of the SBT from Fig 6.7 to the cellular array. We map T1 on column 1 of the array; T2 to column 2 and continue up to T15. Since there is a directed edge $e_{34}$ from T1 to T2, we connect the cell 4 from column 2 to cell 3 in column 1 . Similarly, there is a directed edge between $\mathrm{T} 1$ and $\mathrm{T} 7$, so we connect them by using the cells in Row 1 between cell ' 0 ' and cell '2' as "routing cells". The "routing cells" are indicated by 'R'. It can be observed from Fig 6.7, that one advantage of SBT approach is that we can predict easily what will be the area of the rectangle enclosing our design. 


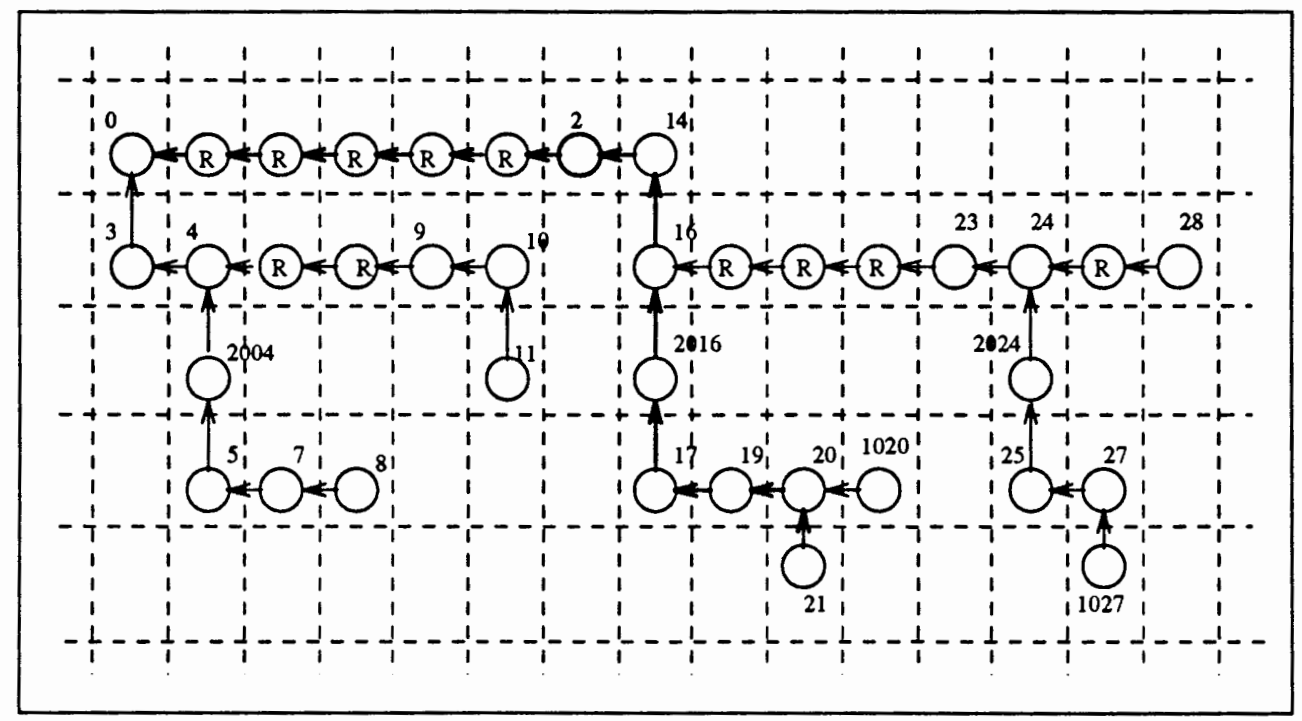

Figure 6.8 Mapping from SBT to the Cellular Array

Each node of the SBT will be implemented in one column of the CA-type array. The number of nodes of SBT is equal to the number of columns of the CA-type array used for design implementation, and the maximum number of vertices of the grouped PRM tree which are projected into one node of the SBT determines the number of rows required.

Using this approach the signal delays can be calculated before performing the actual mapping. We know that in FPGAs all cells have approximately the same delay. Assuming that the delay per each cell is 1 unit, the delay from cell ' 8 ' to the output cell '0' is 7 units. Further, area and delays can be optimized by choosing the proper order of the nodes to be mapped on the array. For example let us consider the path from cell ' 21 ' to cell ' 0 ' in Fig. 6.8. The delay of this path is 14 units and let us assume further that it is a critical path. By changing the order of the node collapsing when SBT is formed we can get new order of the veritices $\mathrm{T} 15, \mathrm{~T} 14 \ldots \mathrm{T} 7, \mathrm{~T} 1, \mathrm{~T} 2 \ldots \mathrm{T} 6$. Using this new order the new mapping is obtained as shown in Fig 6.9. 


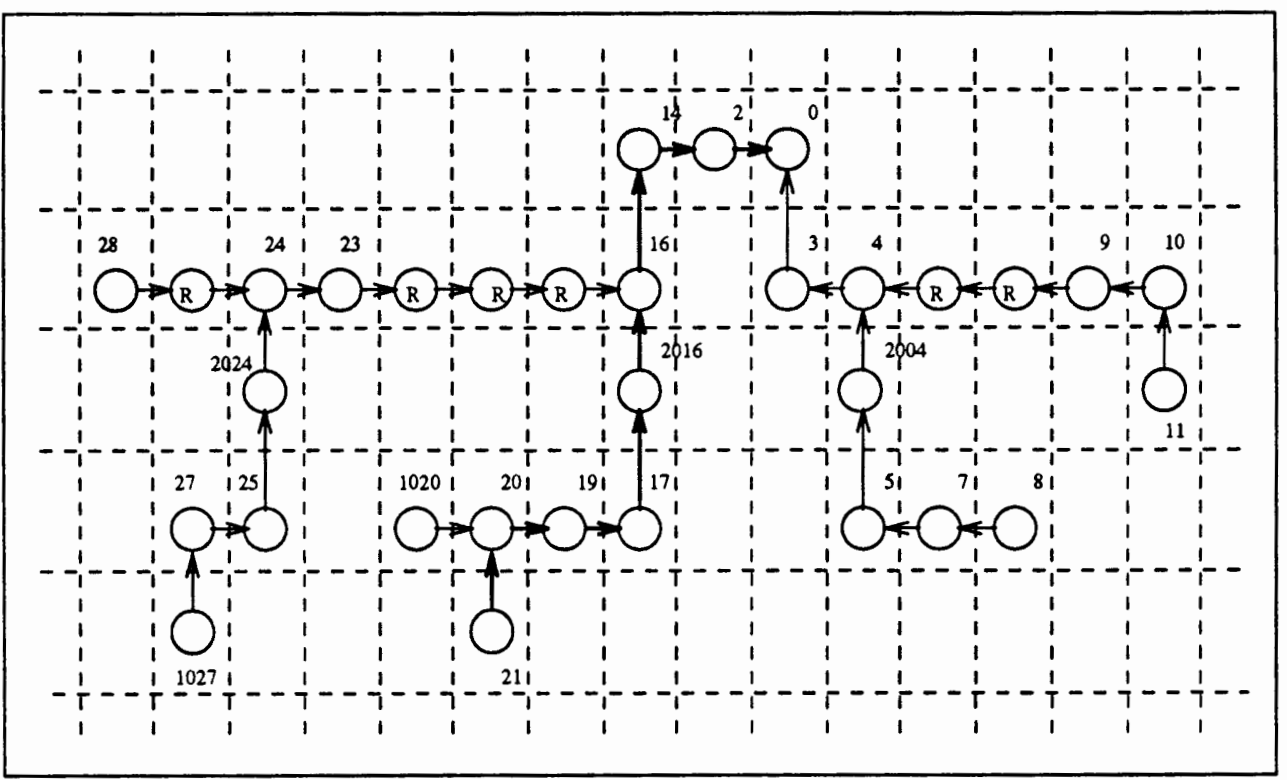

Figure 6.9 Critical Path Control

We can observe that delay in the path from cell ' 21 ' to cell ' 0 ' is reduced from 14 units to 9 units. Therefore, by adding the ordering heuristic we can minimize the length of the critical paths. The predictability of the signal delays is a very important advantage of this approach. 


\subsection{MODIFIED SQUASHED BINARY TREE}

To obtain a more compact shape of the design the original SBT algorithm was modified and the Modified SBT (MSBT) algorithm was implemented in our package. The modified squashed binary tree is formed by projecting nodes of the binary tree onto its leaves in the depth-first manner. By allowing to choose which child node can be used for projection (i.e left most descendant constraint is relaxed) much better optimization was achieved.

The modified squashed binary Tree $T_{b}\left(V_{b}, E_{b}\right)$ consists of the set of vertices $V_{b}$ and the set of directed edges $\mathrm{E}_{\mathrm{b}}$.

$* V_{b}=\left\{v_{b} \mid v_{b}\right.$ represents the vertices of the Tree $T(V, E)$, projected onto the same leaf $\}$ ${ }^{*} E_{B}=\left\{e_{b} \mid e_{b}\right.$ represents the directed edge from $v_{b i}$ to $v_{b j}$ if any of the vertices of the Tree $T(V, E)$ which were collapsed to vertex $v_{b i}$ is connected to any of the vertices of the Tree $T(V, E)$ which were collapsed to vertex $\left.v_{b j}\right\}$. Here the directed edge represents the direction of signal flow.

The MSBT of the PRM tree from Fig 6.6 is shown in Fig 6.10.

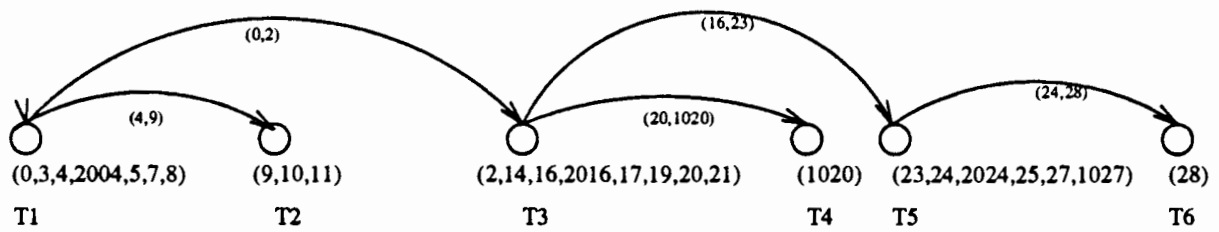

Figure 6.10 Modified Squashed Binary Tree (MSBT)

Mapping the MSBT to the cellular array is done in the same way as was explained for SBT previously. Fig.6.11 shows the mapping of the MSBT from Fig. 6.10 to the cellular array. 


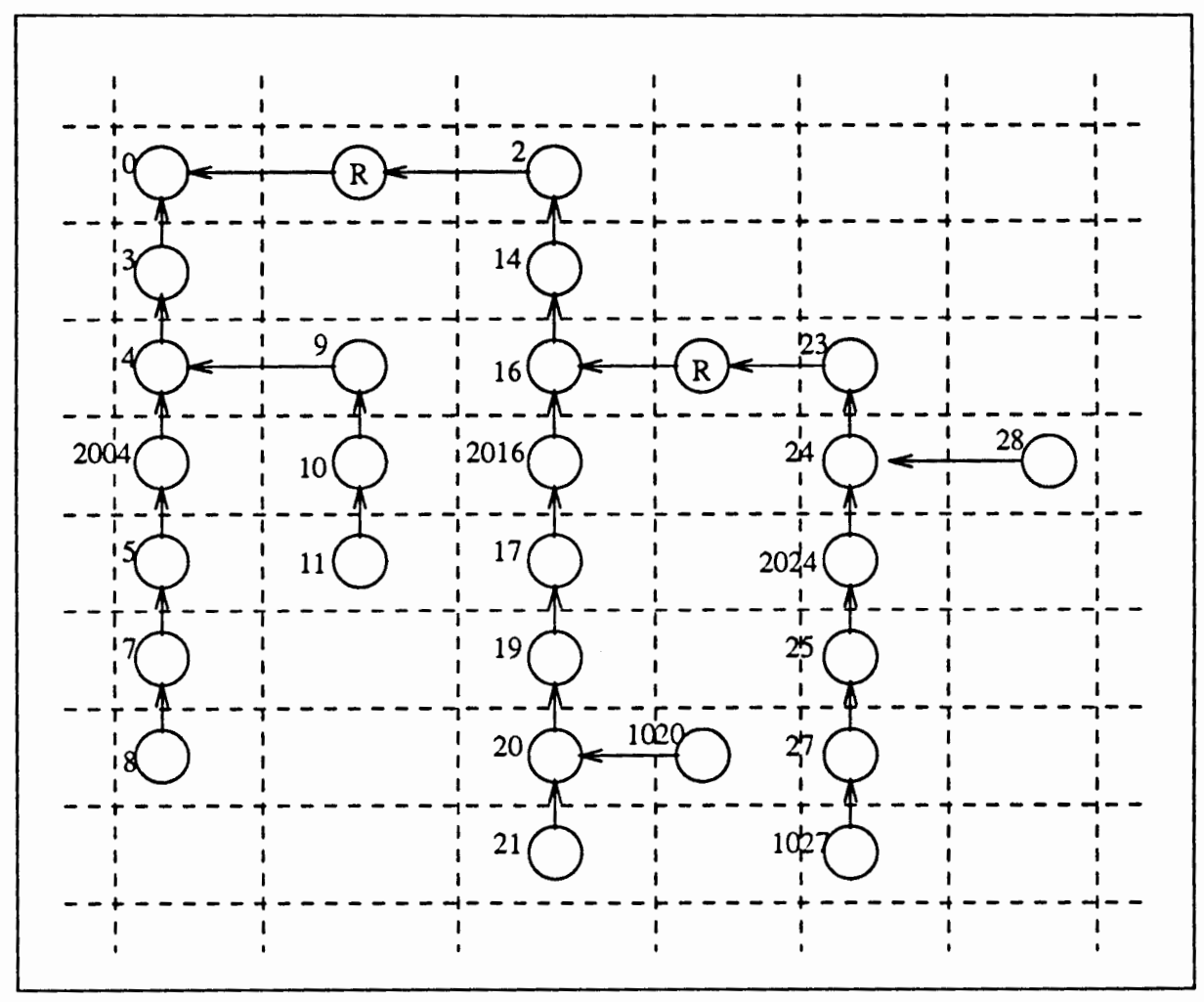

Figure 6.11 Mapping MSBT to the Cellular Array

\subsection{BUS ASSIGNMENT}

Once MSBT is created it can be directly mapped to the CA-type array, and only the necessary logic blocks required for routing need to be added. The exact number and the exact locations of these additional routing blocks is defined by the MSBT structure and hence is known a priori. Then, to complete all connections, the bus assignment has to be performed. The primary (or decomposition) variables from the original PRMT have to be assigned to the local buses. An efficient heuristic has been developed which assigns the variables to the local buses such that the number of local buses used by the same variable and the number of cells needed to distribute the same signal to the different buses are minimized. Each cell in ATMEL 6000 has access to 
four local buses, but only one of them can be used as an input. Once a signal is put on a bus that signal can be used only by the logic blocks in that particular row or column whichever the case may be. Bus assignment algorithm described below uses a greedy approach. Using this approach a feasible bus assignment for all examples has been achieved with a good utilization of bus resources. The steps of the bus assignment algorithms are given below.

Step a: For each decomposition variable $d_{i}$; calculate $M_{i}$, the total number of vertices in PRMT which the variable $d_{i}$ is assigned to. Form a list 'L' of variables for which bus assignment has to be done. Initially this list contains all the primary variables.

Step b: For all $d_{i}$; calculate $R_{i j}$ the total number of nodes of the MSBT named with variable $d_{i}$ and placed in row $r_{j}$ of the cellular array. If $M_{i}=R_{i j}$; then assign variable $d_{i}$ to the upper local bus $U R_{j}$ of the row $R_{j}$. If another variable is already assigned to $U R_{j}$ then assign $d_{i}$ to the bottom local bus $B R_{j}$ of the row $R_{j}$. Remove $d_{i}$ from the list ' $L$ '. If $B R_{\mathrm{j}}$ is occupied then take the next variable from the list. If the list is empty, EXIT.

Calculate $C_{i j}-a$ total number of nodes of the MSBT named with variable $d_{i}$ and placed in column $C_{j}$ of the cellular array. If $M_{i}=C_{i j}$; then assign variable $d_{i}$ to the left local bus $L C_{j}$ of the column $C_{j}$. If another variable is already assigned to $L C_{j}$ then assign $d_{i}$ to the right local bus $R C_{j}$ of the column $C_{j}$. Remove $d_{i}$ from the list. If $R C_{j}$ is occupied then go to Step c. If the list is empty, EXIT.

Step c: Calculate $N_{i}$ for each variable $d_{i}$ in the list, where $N_{i}=M_{i} / 2$ (if $M_{i}$ even); $N_{i}=\left(M_{i} / 2\right)+1$ (if $M_{i}$ odd). Substitute $M_{i}=N_{i}$.

Step d: For all $d_{i}$; If $M_{i} \geq R_{i j}$, then assign $d_{i}$ to $U R_{j}$. If $U R_{j}$ is already assigned 
then assign the variable to $B R_{j}$. Decrement $M_{i}$ by $R_{i j}$. If $M_{i}=0$; Remove $d_{i}$ from the list. If $\mathrm{BR}_{\mathrm{j}}$ is occupied then take the next variable from the list. If the list is empty then, EXIT. If $M_{i} \geq C_{i j}$; then assign $d_{i}$ to $L C_{i}$. If $L C_{i}$ is already assigned then assign the variable to $R C_{j}$. Decrement $M_{i}$ by $C_{j}$. If $M_{i}=0$; remove $d_{i}$ from the list. If $R C_{j}$ is occupied then go to Step c.

If the list is empty, EXIT. If the list is not empty, then Step e.

Step e: If the list is not empty, repeat Step c and Step d until all connections are completed.

The completed mapping of the MSBT of our leading example to the ATMEL 6000 series FPGA is shown in Fig. 6.12.

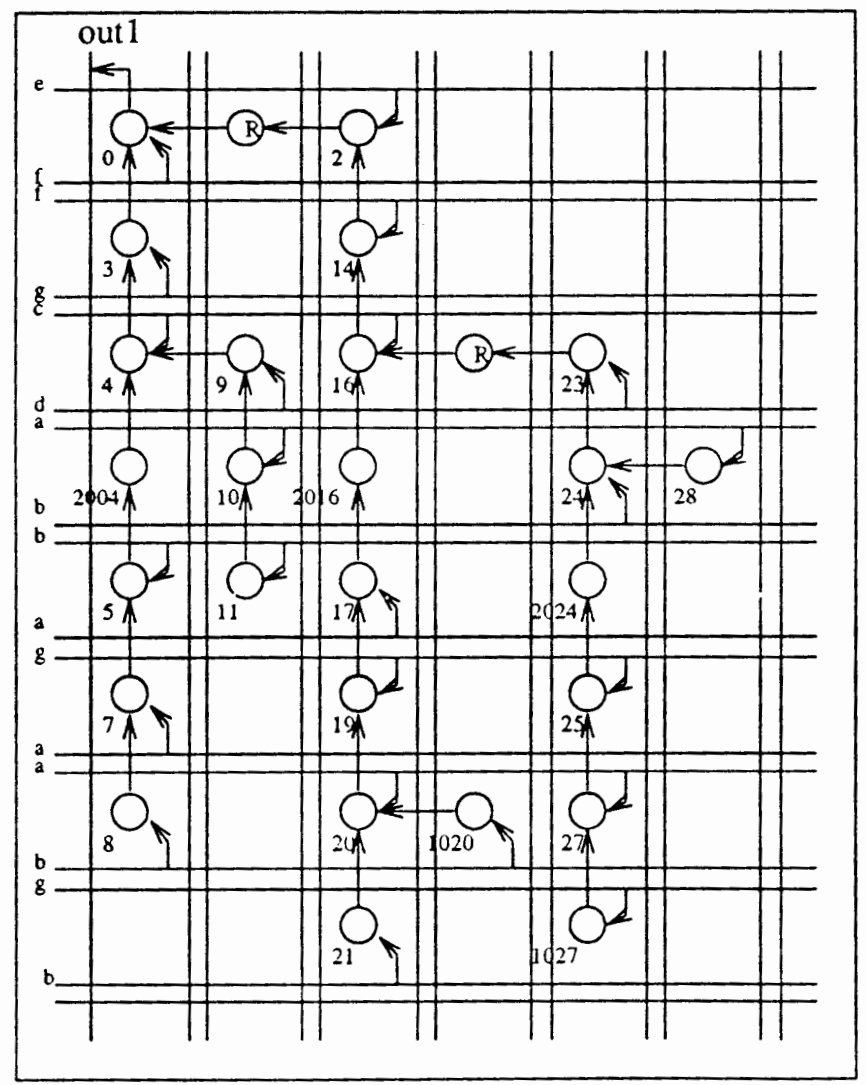

Figure 6.12 Final Mapping of MSBT with Bus Assignment 


\subsection{MULTI-OUTPUT FUNCTIONS}

The above discussed algorithms from the previous sections 6.3 and 6.4 work for multi-input and single-output functions. This restriction comes from the logic synthesis method used. But most of the real circuits are multi-input and multi-output functions, which are usually represented as a directed acyclic graph (DAGs). Therefore, one possible approach would be to decompose the DAG into a number of trees. This can be done using for example the cone partitioning method developed in [18]. The cone partitioning method was developed taking into account the I/O limitations of FPGAs and the critical paths within the circuit partitions.

\subsection{COMPARISON OF OUR METHOD WITH OTHER METHODS}

DAG-Approach: In the general DAG mapping method developed in [12], a Boolean function is modeled as DAG, where each node represents the logic which can be realized in one logic block of the target architecture and an edge represents the connectivity between the cells. All the cells connected to the same primary input variable are placed on a single column of the target architecture using the linear column ordering method. The final placement is further tuned using an iterative improvement based approach (Simulated Evolution) [12].

In this method the initial placement is done using alternate columns to place the grouped cells, in order to accomodate routing cells later in the iterative improvement phase. This leads to a lot of unused cells, and they are considered to be wasted when the rectangular area of the mapped circuit is taken into account.

The iterative method based on Simulated Evolution is a non-deterministic algorithm, in the event of an incremental design change this method is disadvantageous as the routing paths change due to different placement and hence the timing of the entire circuit may change. 
To reduce the complexity of placement only vertical buses were used to assign the input variables in this method. However, the $\mathrm{I} / \mathrm{O}$ pads are usually placed on both horizontal and vertical boundaries of FPGAs, therefore if a variable has to come from horizontal boundary the number of cells used as wires increase, and the throughput of the chip is reduced.

Two-Dimensional Array Approach: This method [11] synthesizes a given circuit in the form of multi-level SOP/ESOP form. A set of functions realizable by each of the logic cells is limited to a finite set of simple logic gates which includes an EXOR gate. The CA-type FPGA is represented as two distinct planes, input plane (columns of logic cells represent multi-level terms) and output plane (collecting terms), as shown in Fig. 6.13

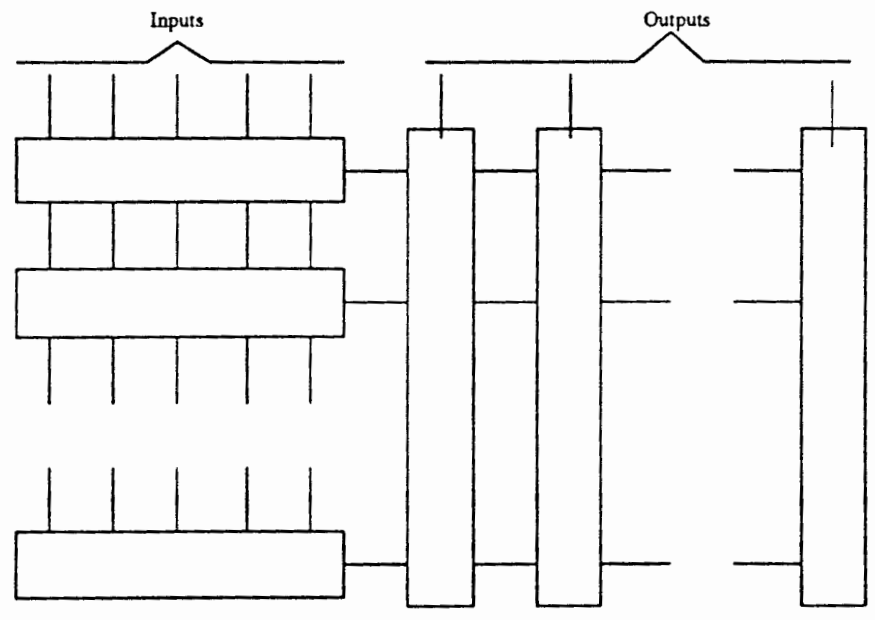

Figure 6.13 CMLA: Two-Dimensional Array

A string of literals connected by a set of operators in which no literal appears more than once is defined as a complex term The operators in a complex term can be either AND, OR, EXOR or their complements. All operations in a complex term, must be performed in a sequence from left to right or from right to left and all 
operators have the same priority.

For example,

$$
(a \bar{b}+c),(a+b) c
$$

are two complex terms, when the operations are performed from left to right.

In a CA-type FPGA, due to a local connectivity between adjacent blocks, a complex term can be realized using a column of logic cells and primary variables are assigned to local buses, one per each column of cells. Let us consider the example from [12]. The result from the factorization phase is:

$$
\begin{aligned}
& f_{0}=(b d+a) c+b d a \quad \text { which is two complex terms; } \\
& f_{1}=b d \quad \text { which is one complex term; } \\
& f_{2}=b d \quad a c \quad \text { which is one complex term. }
\end{aligned}
$$

Fig. 6.14 shows the realization of the above complex terms with setting the variable order as $(b, d, a, c)$. One advantage of this approach is that no separate routing step is required to realize a complex term [12].

This method was developed for generic CA-type FPGA. However, if we attempt a realization with ATMEL 6000 [2] FPGA as a target architecture, we see a Type I mismatch in the last row (EXOR-EXOR), in Fig. 6.14. To avoid the mismatch we must add a switch cell between the two EXOR gates. It means that we have to move the entire column, associated with the expansion varible " $c$ " to the next column. In the ATMEL architecture AND-EXOR gate combination can be realized in one cell. The CMLA method does not take advantage of this powerful feature. As a result of that the additional cell used increases the delay of the design. Thus the developement of a generalized method should be followed by device specific technology mapping technique. 


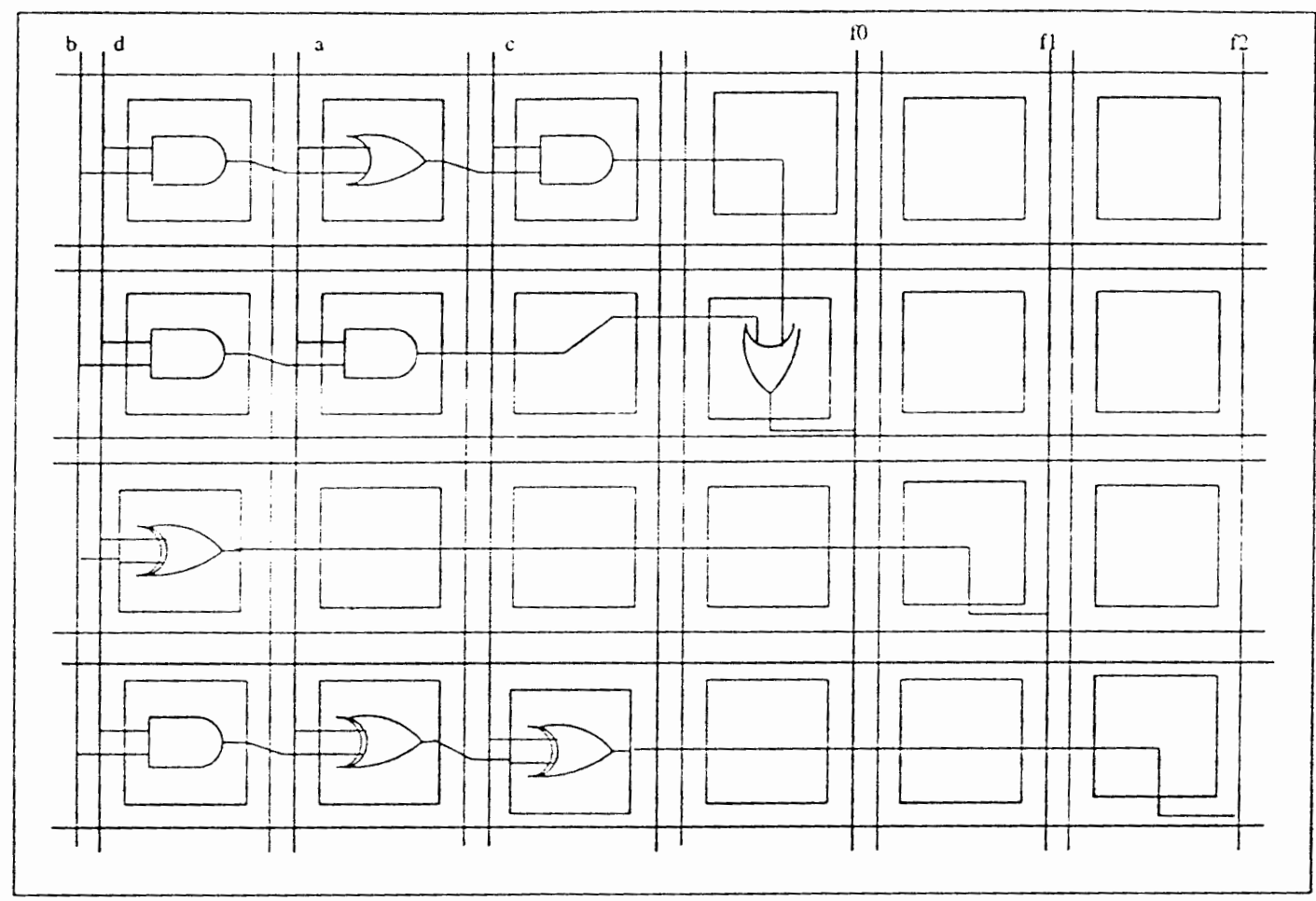

Figure 6.14 CMLA: Realization of a Function

Macro-Generator Approach: The current industrial method (ATMEL) uses Macro-Generators approach, where basic modules are provided in the library and automatic placement routing techniques are used for placing and connecting modules. This method does not provide any opporunity for synthesis of general purpose functions where decompositions into submodules are not known. Secondly, the modules have irregular shapes and routing requires many cells to be used just for connections. Therefore, it is difficult to achieve a good performance of the implemented circuits. 


\section{CHAPTER VII}

\section{RESULTS}

TRM was applied to a set of MCNC benchmarks. Since TRM is applicable to single output functions, we modified MCNC benchmarks to extract single output functions. We compare the area and the number of local buses used by Squashed Binary Tree (SBT) method versus Modified Squashed Binary Tree (MSBT) approach, and MSBT approach versus commercially available ATMEL (IDS) tools. We assume ATMEL 6000 as the target architecture. The results are presented in Table I and Table II.

Table I shows the results of the SBT and MSBT approaches for the modified set of MCNC benchmarks. The second column, "PRMT", shows the number of gates required to implement the function in PRMT form (tree generated by REMIT).

In the GROUPING section of the Table I, column "L" shows the number of logic blocks used to implement the logic and column "C" shows the number of connecting cells added due to A-B restrictions of the ATMEL architecture [2]. Column "GT" shows the total number of cells. SBT and MSBT column sections present the results of SBT and MSBT approaches, respectively. $R$ is the number of routing logic blocks added when constructing SBT or MSBT. T is the total number of logic blocks required to realize the function. RT represents the size (in terms of number of cells) of the smallest rectangle enclosing the mapped circuit. The results clearly show that MSBT approach has significantly reduced the total number of logic blocks required to implement a given function and the size of the enclosing rectangle is also smaller. 
Table 1: Cell Utilization SBT Vs. MSBT

\begin{tabular}{|c|c|c|c|c|c|c|c|c|c|c|}
\hline \multirow{2}{*}{ MCNC } & \multirow{2}{*}{$\begin{array}{c}\text { PRMT } \\
\text { \# of nodes } \\
\end{array}$} & \multicolumn{3}{|c|}{ GROUPING } & \multicolumn{3}{|c|}{ SBT } & \multicolumn{3}{|c|}{ MBST } \\
\hline & & L & $\mathrm{C}$ & GT & $\mathrm{R}$ & $T$ & RT & $\mathrm{R}$ & $\mathbf{T}$ & RT \\
\hline $5 \times 1$ & 29 & 21 & 2 & 23 & 11 & 34 & 75 & 2 & 25 & 48. \\
\hline $5 \times 10$ & 17 & 15 & 0 & 15 & 5 & 20 & 26 & 0 & 15 & 21 \\
\hline $5 \times 5$ & 11 & 7 & 1. & 8 & 4 & 12 & 21 & 0 & 8 & 14 \\
\hline لـ لـمع & 18 & 15 & 7 & 22 & 8 & 30 & 84 & 1 & 23 & 36 \\
\hline con 12 & 12 & 6 & 3 & 9 & 2 & 11 & 28 & 0 & 11 & 18 \\
\hline datalo & 443 & 222 & 74 & 301 & 44 & 345 & 1092 & 44 & 345 & 1052 \\
\hline data 14 & 23 & 80 & 8 & 88 & 88 & 176 & 402 & 12 & 100 & 182 \\
\hline fl5 & 249 & 151 & 21 & 172 & 79 & 251 & 783 & 36 & 208 & 432 \\
\hline f16 & 189 & 107 & 5 & 112 & 91 & 203 & 568 & 26 & 138 & 308 \\
\hline$f 18$. & 423 & 272 & 14 & 286 & 313 & 599 & 1930 & 32 & 28 & 1140 \\
\hline f19 & 634 & 443 & 241 & 684 & 541 & 1225 & 4424 & 200 & 893 & 3072 \\
\hline 65 & 11 & 8. & 0 & 8 & 0 & 8 & 12 & 0 & 8 & 12 \\
\hline misex 20 & 795 & 412 & 140 & 552 & 173 & 725 & 2288 & 173 & 725 & 2272 \\
\hline $\operatorname{misex} 21$ & 25 & 17 & 2 & 19 & 7 & 26 & 52 & 1 & 20 & 54 \\
\hline -misex 22 & 20 & 16 & 1 & 17 & 5 & 22 & 44 & 0 & 17 & 24 \\
\hline misex 23 & 19 & 13 & 1 & 14 & 4 & 18 & 36 & 0 & 14 & 27 \\
\hline misex 25 & 20. & 16 & 5 & 21 & 3 & 24. & 66 & 1 & 21 & 45 \\
\hline -misex 26 & 14 & 12 & 4 & 16 & 3 & 19 & 40 & 0 & 16 & 24 \\
\hline $\operatorname{misex} 48$ & 25 & 16 & 1 & 17 & 6 & 23 & 48 & 0 & 17 & 45 \\
\hline $\operatorname{misex} 49$ & 20 & 16 & 1 & 17 & 5 & 22 & 36 & 0 & 17 & 21 \\
\hline misex 50 & 21 & 14. & 1. & 15 & 5 & 20 & 40 & 1 & 16 & 32 \\
\hline misex 52 & 20 & 16 & 1 & 23 & 4 & 27 & 66 & 1 & 24 & 45 \\
\hline misex 53 & 15. & 10 & 2 & 12 & 2 & 14 & 24 & 1 & 13 & 24 \\
\hline -misex 54 & 24 & 18 & 4 & 22 & 5 & 27 & 48 & 1 & 23 & 35 \\
\hline misex 55 & 21 & 17 & 5 & 22 & 7 & 29 & 52 & 1 & 23 & 35 \\
\hline $\operatorname{misex} 56$ & 21. & 14 & 1 & 15 & 5 & 20 & 40 & 1 & 16 & 32 \\
\hline misex 57 & 20 & 17 & 3 & 20 & .5 & 25 & 65 & 1 & 21 & 28. \\
\hline $\operatorname{misex} 58$ & 13 & 10 & 7 & 17 & 3 & 20 & 48 & 1 & 18 & 36 \\
\hline $\operatorname{misex} 60$ & 68 & 61 & 11 & 72 & 69 & 141 & 378 & 5 & 77 & 112 \\
\hline misex 61 & 70 & 63 & 11. & 74 & 73 & 147 & 392 & 5 & 79 & 120 \\
\hline $\operatorname{misex} 62$ & 276 & 394 & 131 & 525 & 173 & 728 & 2240 & 173 & 698 & 2208 \\
\hline $\operatorname{misex} 63$ & 845 & 446 & 148 & 594 & 183 & 277 & 2736 & 183 & 777 & 2704 \\
\hline $\operatorname{misex} 64$ & 443 & 227 & 74 & 301 & 44 & 345 & 1092 & 44 & 345 & 1050 \\
\hline $\operatorname{san} 21$ & 551 & 337 & 105 & 442 & 186 & 628 & 2016 & 151 & 593 & 172 \\
\hline $\operatorname{san} 22$ & 779 & 408 & 08 & 516 & 181 & 607 & 2295 & 175 & 691 & 2025 \\
\hline $\operatorname{sac} 23$ & 846 & 475 & 125 & 600 & 205 & 805 & 2450 & 205 & 805 & 2422 \\
\hline majority & 20 & 15 & 5 & 20 & 6 & 26 & 44 & 0 & 20 & 32 \\
\hline mux & 97 & 83 & 83 & 160 & 19 & 179 & 570 & 13 & 173 & 1480 \\
\hline
\end{tabular}


To illustrate the differences between our final layout and the layout generated by ATMEL tools we have presented one detailed example. In Fig. 3.2 the View Logic schematic of the MCNC 5X1 benchmark represented as a PRMT was shown. The ATMEL generated layout for that example is shown in Fig. 7.1, and the layout generated by our TRM package in Fig. 7.2. It can be easily noticed that our layout is more compact and gives the better utilization of the chip resources and therefore gives us better performance. In Table II the comparison between our method Tree Restructuring Mapping (TRM) and the ATMEL commercial tools package (IDS) is presented using the MCNC benchmarks. $\mathrm{B}$ is the number of buses and $\mathrm{L}$ is the number of logic blocks used for implementing the logic, $C$ is the number of cells used for routing, and $A$ represents the rectangular area occupied by the core of the design (without $\mathrm{I} / \mathrm{O}$ pins). We compare only core area of the mapped design because the ATMEL tools perform bus assignment in an inefficient way, and the resulting area is very large and contains a lot of unused logic cells. As it can be seen from the Table II our methods give much more compact layouts than the ATMEL tools. The number of local buses and logic blocks used for routing is much smaller for all run examples. The $L / R$ ratio, logic blocks to routing blocks, is high for our method, and it gives more "logic power" to the implementation of the designs and improves performance.

Table II Area Comparison TRM Vs. ATMEL (IDS)

\begin{tabular}{|c|c|c|c|c|c|c|c|c|}
\hline \multirow{2}{*}{ MCNC } & \multicolumn{4}{|c|}{ ATMEL(IDS) } & \multicolumn{4}{|c|}{ Our Program(TRM) } \\
\cline { 2 - 10 } & B & L & C & A & B & L & C & A \\
\hline \hline & & & & & & & & \\
\hline $\mathbf{5 X 1 0}$ & $\mathbf{4 6}$ & $\mathbf{1 7}$ & $\mathbf{2 3}$ & $\mathbf{9 0}$ & $\mathbf{1 9}$ & $\mathbf{1 5}$ & $\mathbf{6}$ & $\mathbf{2 7}$ \\
\hline f5 & 28 & 11 & 12 & 45 & 10 & 8 & 6 & 12 \\
\hline misex54 & 58 & 24 & 27 & 180 & 19 & 18 & 13 & 35 \\
\hline & & & & & & & & \\
\hline
\end{tabular}


To compare the performance improvement we have done timing analysis using ATMEL (IDS) package. The design, generated by our TRM package is entered using interactive editor of the ATMEL (IDS) tools, and then we run the timing analysis from the ATMEL (IDS) package on both implementaions. The longest path delays obtained is shown are Table III. The average improvement achieved with our approach is around $50 \%$.

Table III Delay Comparison TRM Vs. ATMEL (IDS)

\begin{tabular}{|c|c|c|}
\hline MCNC & ATMEL ns & TRM ns \\
\hline & & \\
\hline $\mathbf{5 X 1 0}$ & $\mathbf{7 1 . 1 4}$ & $\mathbf{2 7 . 9 0}$ \\
\hline $\mathrm{f5}$ & 57.30 & 26.70 \\
\hline misex54 & 107.40 & 36.40 \\
\hline & & \\
\hline
\end{tabular}




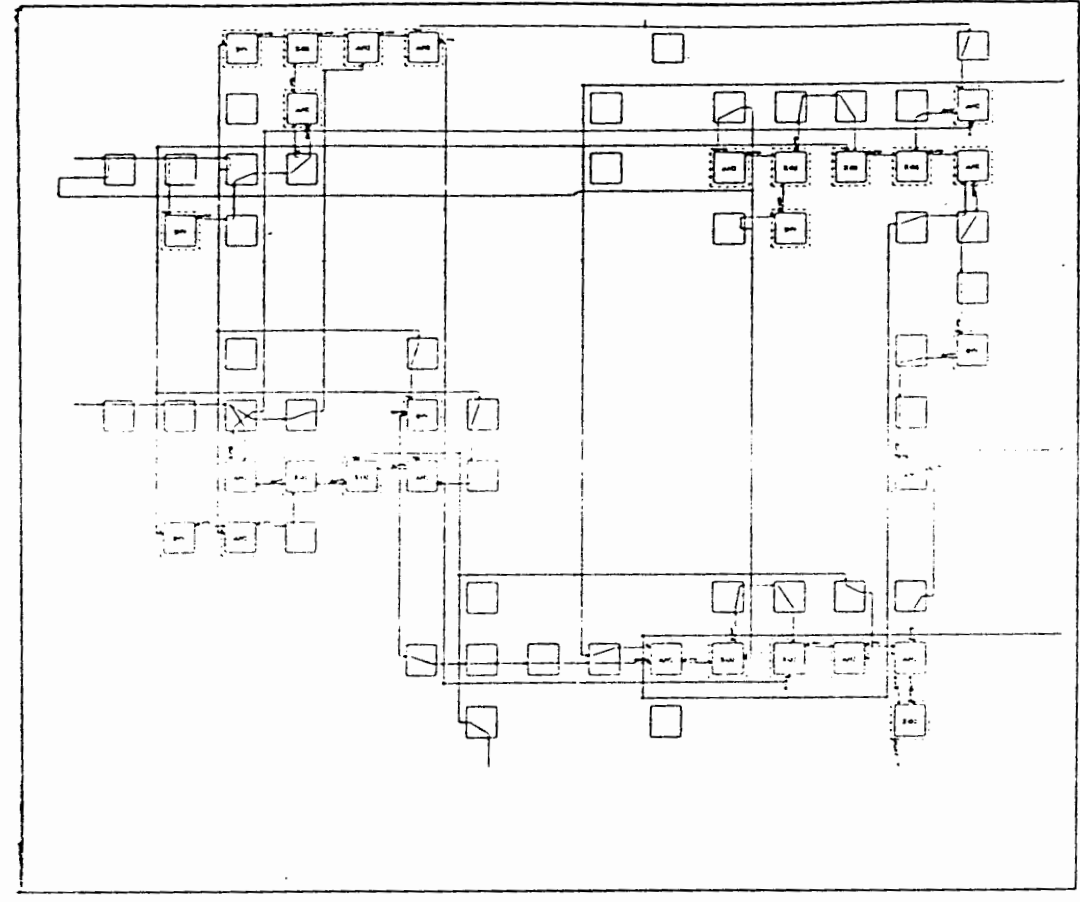

Figure 7.1 Layout of MCNC $5 \times 1$ benchmark generated by ATMEL (IDS) Tools

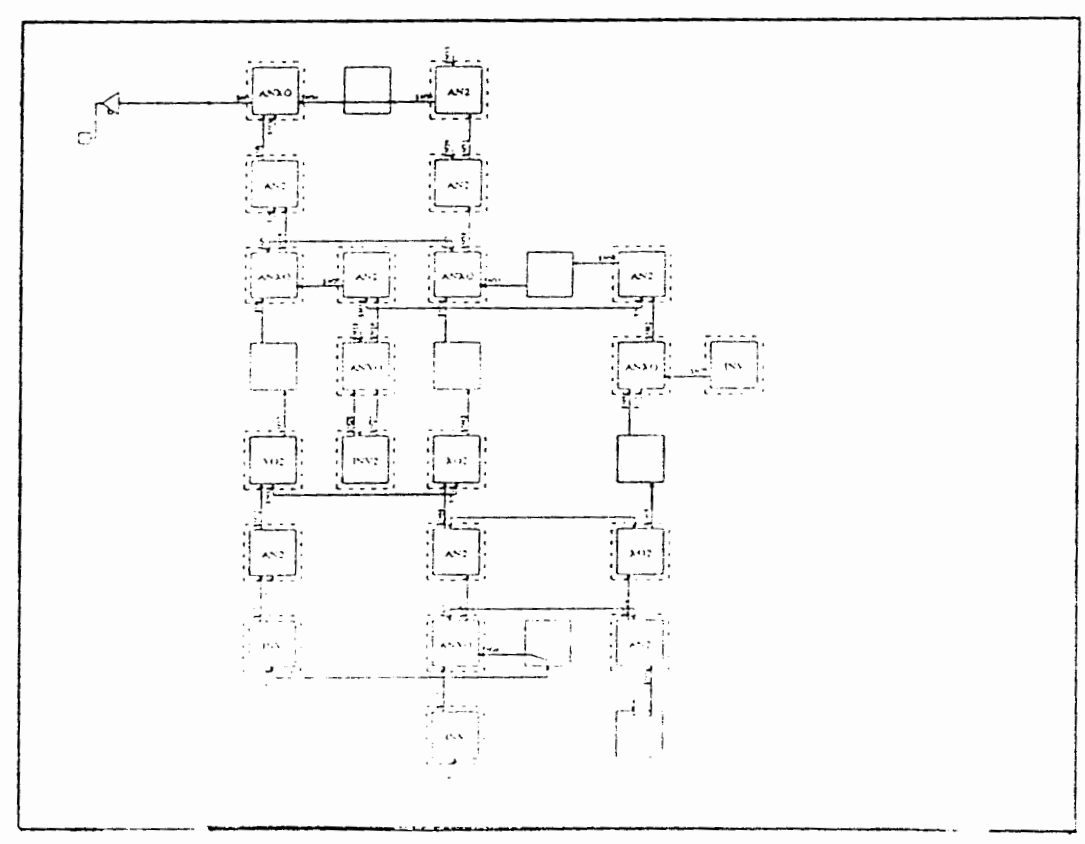

Figure 7.2 Layout of MCNC 5x1 benchmark generated by TRM Package 


\section{CHAPTER VIII}

\section{CONCLUSIONS}

We proposed a new tree restructuring method for mapping combinatorial circuits onto CA-Type FPGAs. Using this approach the routing phase was completely eliminated, by preserving the local connectivity among the logic blocks. Mapping process is straight-forward and therefore enables predictability of the signal delays, which is very important advantage of this method.

Research from $[43,44]$ shows that by allowing various polarities of variables we can obtain trees with fewer nodes than the PRMT, and with proper technology mapping developed for this new methods we can obtain significant improvement in the final layout. Our program TRM is independent of the functionality of the logic gates in in the netlist obtained from logic optimization steps, as long as the function is represented as a binary tree. Our method is a general method and can be applied for a general class of CA-type FPGAs. The results on some MCNC benchmarks shows that our method is better both in area and delay when compared to commercially available tools.

Next phase of work should be towards extending the TRM for multi-output functions. Further improvements can be can achieved by combining together the ordering of the node collapsing in MSBT and the bus assignment procedure. The minimization of the longest path can be used as a constraint in ordering of the collapsing nodes when MSBT is formed. The bus assignment heuristic could also be further improved. 
My contribution in this thesis work, I investigated and implemented the SBT approach as a solution for mapping of a binary tree to Cellular Arrays. Developed and implemented the MSBT and Bus-Assignment algorithms. Developed the framework for VHDL/SCHEMATIC design entry for the TRM package. 


\section{REFERENCES}

[1] L. Wu, and M. A. Perkowski, "Minimization of Permuted Reed-Muller Trees for Cellular Logic Programmable Gate Arrays," In H. Gruenbacher and R. Hartenstein (eds.), LNCS, No. 705, Springer Veriag, pp. 78-87, 1993.

[2] ATMEL Corporation CMOS Integrated Circuit Data Book, 1993:94. 2125 O'Neil Drive, San Jose, CA, 95131.

[3] F. T. Leighton, Introduction to Parallel Algorithms and Architectures: Arrays, Trees, Hypercubes, Morgan Kaufmann Publishers, Inc., San Mateo, CA, 1992.

[4] Z. Songhua, "Grouping of The Permuted Reed-Muller Tree", Report, Portland State University., 1993.

[5] T. Sasao and Ph. Besslich, "On The Complexity of Mod-2 Sum PLA's," IEEE Trans. Comput., vol. 39, No. 2, pp. 262-266, Feb. 1990.

[6] N. Ramineni, and M. Chrzanowska-Jeske, "A Routing-Driven Mapping For Cellular-Architecture FPGA's", 36th Midwest Symposium On Circuits \& Systems pp. 308-311, Aug 1993.

[7] M. Chrzanowska-Jeske, "Architecture and Synthesis Issues in FPGAs," Proc. of Northcon, pp. 102-105, October 1993.

[8] F. Furtek, "An FPGA Architecture for Massively Parallel Computing," 2nd International Workshop on Field Programmable Logic and Applications, August 1992.

[9] N. B. Bhat and D. D. Hill, "Routable Technology Mapping for FPGA's," 1992 ACM First International Workshop on FPGAs, pp. 143-148, 1992. 
[10] R. J. Francis, J. Rose, and Z. Vranesic, "Chortle-crf: Fast Technology Mapping for Lookup Table-Based FPGAs," Proc. 28th DAC, pp. 227-233, June 1991.

[11] A. Sarabi, N. Song, M. Chrzanowska-Jeske, M. Perkowski, "A Comprehensive Approach to Logic Synthesis and Physical Design for Two-dimensional Logic Arrays", Proc. of 31st DAC, pp. 321-326, June 1994.

[12] A. K. Dasari, N. Song, M. Chrzanowska-Jeske, "Layout Driven factorization and fitting for Cellular architecture FPGAs", Proc. of Northcon, pp. 106-111, 1993.

[13] Concurrent Logic, Inc., Seminar at PSU, Nov. 17th, 1992.

[14] U. Kebschull, E. Schubert, W. Rosenstiel, "Multilevel Logic Synthesis Based on Functional Decision Diagrams," Proc. IEEE European Design Automation Conference, pp. 43-47, 1992.

[15] A. Mukhopadhyay, "Cellular Logic," in "Recent Developments in Switching Theory," Ed. Mukhopadhyay, A., pp. 281-285, 1971.

[16] M. A. Perkowski, A. Sarabi, and F. R. Beyl, "XOR Canonical Forms of Switching Functions," Proc. of the IFIP WG 105 Workshop on Applications of the ReedMuller Expansion in Circuit Design, Hamburg, Germany, pp. 27-32. September 1993.

[17] I.Schaefer, M.A.Perkowski, H.Wu, "Multilevel Logic Synthesis for Cellular FPGAs Based on Orthogonal Expansions," Proc. IFIP WG 10.5 Workshop on Applications of the Reed-Muller Expansion in Circuit Design. Hamburg, Germany, pp. 42-51, Sept. 1993.

[18] G. Saucier, D. Brasen, J.P. Hiol. "Partitioning With Cone Structures," Proc. IEEE ICCAD 93, pp. 236-239, 1993. 
[19] R.L. Ashenhurst, "The Decomposition of Switching Functions," Proc. Int'l Symp. Theory of Switching Functions, pp. 74-116, 1959.

[20] H. A. Curtis, "Generalized Tree Circuit - The Basic Building Block of an Extended Decomposition Theory," Journal of ACM, vol. 10, pp. 562-581, 1963.

[21] M. Davio, J. P. Deschamps, and A. Thayse, in Discrete and Switching Functions, McGraw-Hill, 1978 .

[22] M. A. Perkowski and P. D. Johnson, "Canonical Multi-Valued Input Reed-Muller Trees and Forms," 3rd NASA Symposium on VLSI Design, 1991.

[23] S. B. Akers, "A Rectangular Logic Array," IEEE Trans. on Comput. C-21, No. 8, pp. 848-857, 1972.

[24] V. Dvorak, "A Two-Rail Cascade Synthesis of Boolean Functions," IEEE Trans. on Comput. vol. C-17, No. 6, pp. 592-596, 1968.

[25] B. Elspas, The Theory of Multirail Cascades, Recent Developments in Switching Theory, Ed. A. Mukhopadhyay, Academic Press, pp. 315-367, 1971

[26] S. N. Kukreja and I. Chen, "Combinational and Sequential Cellular Structures," IEEE Trans. on Comput. C-22, No. 9, pp. 813-823, 1973.

[27] M. K. K. Maitra, "Cascaded Switching Networks of Two-Input Flexible Cells," IRE Trans. Electron. Comput. EC-11, pp. 136-143, 1962.

[28] R. C. Minnick, "Cutpoint Cellular Logic," IEEE Trans. on Electron. Comput. EC-13, pp. 685-698, 1964.

[29] R. C. Minnick, "Cobweb Cellular Arrays," AFIPS Conf. Proc. 1965.

[30] R. C. Minnick, "A Survey of Microcellular Research," J. of ACM, vol. 14, No. 2, pp. 203-241, 1967. 
[31] A. Mukhopadhyay,"Unate Cellular Logic," IEEE Trans. on Comput. vol. C-18, No. 2, pp. 114-121, 1969.

[32] A. Mukhopadhyay and H. S. Stone, "Cellular Logic," in Recent Developments in Switching Theory, Ed. A. Mukhopadhyay, Academic Press, pp. 281-285, 1971.

[33] R. A. Short,"Two-Rail Cellular Arrays," AFIPS Conf. Proc. vol. 27, pt. 1, pp. $355-369,1965$.

[34] H. S. Stone and A. J. Korenjak, "Canonical Form and Synthesis of Cellular Cascades," IRE Trans. Electron. Comput. vol. EC-14, No. 6, pp. 852-862, 1965.

[35] S. Swamy, "On Generalized Reed-Muller Expansion," IEEE Trans. on Comput. vol. C-21, pp. 1008-1009, 1972.

[36] M. Yoeli, "Group-Theoretic Approach to Two-Rail Cascades," IRE Trans. Electron. Comput. vol. EC-14, pp. 815-822, 1965.

[36] K. Chaudhary and M. Pedram, "A Near Optimal Algorithm for Technology Mapping Minimizing Area under Delay Constraints," Proc. 29th ACM/IEEE Design Automation Conf., pages 492-98, 1992.

[37] K. Karplus, "Xmap: A Technology Mapper for Table-Lookup Field-Programmable Gate Arrays." In Proceedings of the Design Automation Conference, pages $240-243,1991$.

[38] Jason Cong and Yuzheng Ding, "On Area/Depth Trade-off in LUT-Based FPGA Technology Mapping," 30th ACMIIEEE DAC. pp. 213-218. 1993.

[39] Jason Cong and Yuzheng Ding, "An Optimal Technology Mapping Algorithm for Delay Optimization in Lookup-Table Based FPGA Designs," Proc. IEEE ICCAD, pp. 48- 53, November 1992. 
[40] A. Bedarida, S. Ercolani, and G. DeMicheli, "A New Technology Maping Algorithm for the Design and Evaluation of Fuse/Antifuse-based Field-Programmable Gate Arrays," Ist ACM Workshop on FPGAs, pp. 103-108, Berkeley, CA, February 1992.

[41 Algotronix Lid., Configurable Array Logic User Manual, Edinburgh, UK, 1991.

[42] Motorola Inc., MPA1OXX Field Programmable Gate Arrays Product Brief, USA, 1993

[43] A. Sarabi, P. F. Ho, K. Iravani and M. A. Perkowski, "Minimal Multi-level Realization of Switching Functions Based on Kronecker Functional Decision Diagrams," IEEE Int. Workshop Logic Synthesis, pp. P3-1, 1993.

[44] P. F. Ho, M. A. Perkowski, "Free Kronecker Decision Diagrams and their Application to ATMEL 6000 FPGA Mapping," Proc. Euro-DAC'94 with Euro-VHDL'94, pp. 8-13, September 19-23, 1994, Grenoble France.

[45] N. Song and M. A. Perkowski, "EXORCISM-MV-2: Minimization of Exclusive Sum of Products Expressions for Multiple-Valued Input Incompletely Specified Functions," Proc. of IEEE Int. Symp.on Multi-Valued Logic, pp. 132-137, 1993.

[46] T. Sasao, "EXMIN 2: A Simplification Algorithm for Exclusive-Sum-Of-Products Expressions for Multiple-Valued-Input Two-Valued-Output Functions," IEEE Trans. on $C A D$, vol. 12, No. 5, pp. 621-632, 1993.

[47] ATMEL Corporation, Integrated Development System, Macro Library I and II, Version 1.10, Release Series 1.10c, August 1993. 2125 O'Neil Drive, San Jose, CA, 95131. 


\section{APPENDEX A}

(Layouts of MCNC Benchmarks f5,5x10,misex 54) 


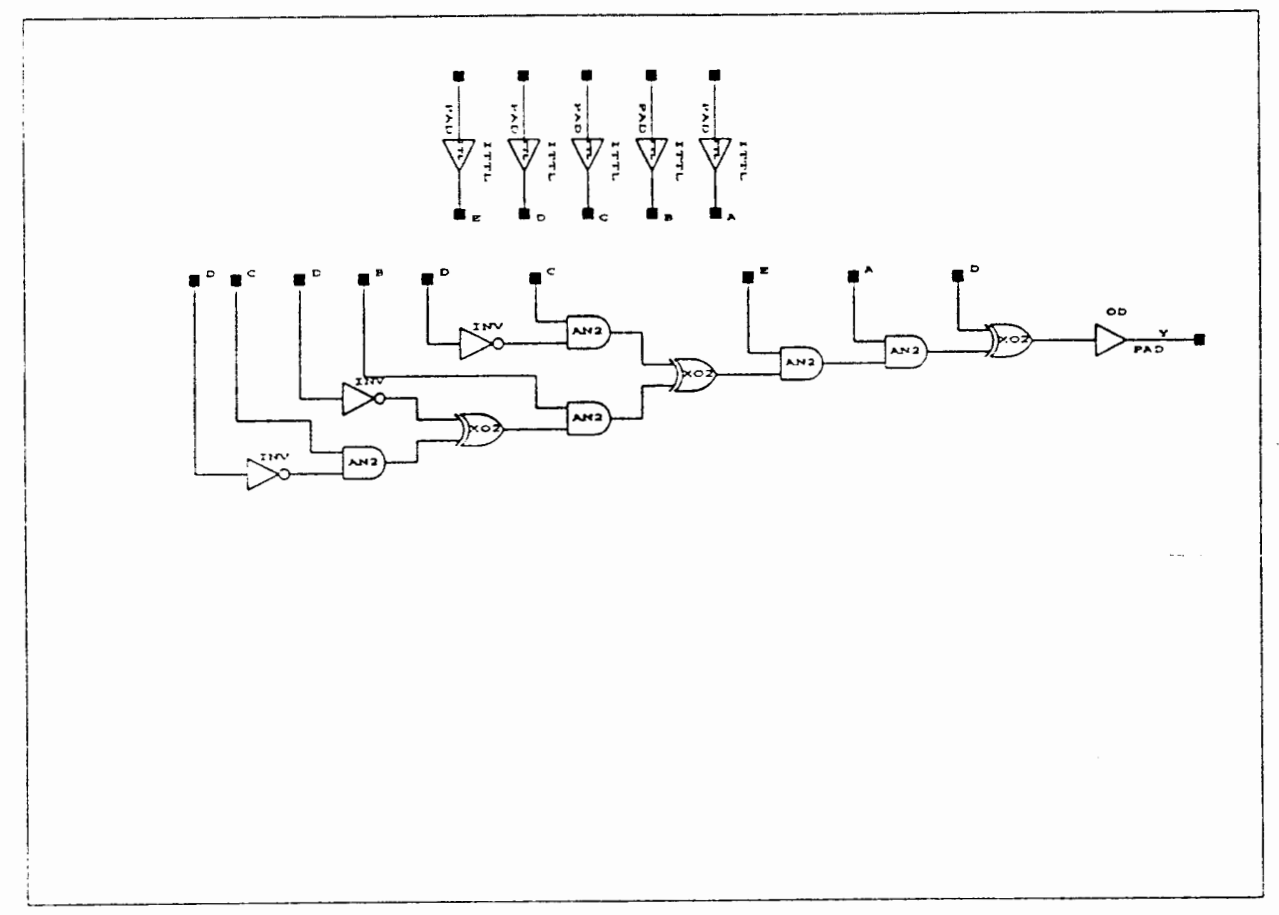

Figure A.4 ViewLogic Schematic of MCNC f5 


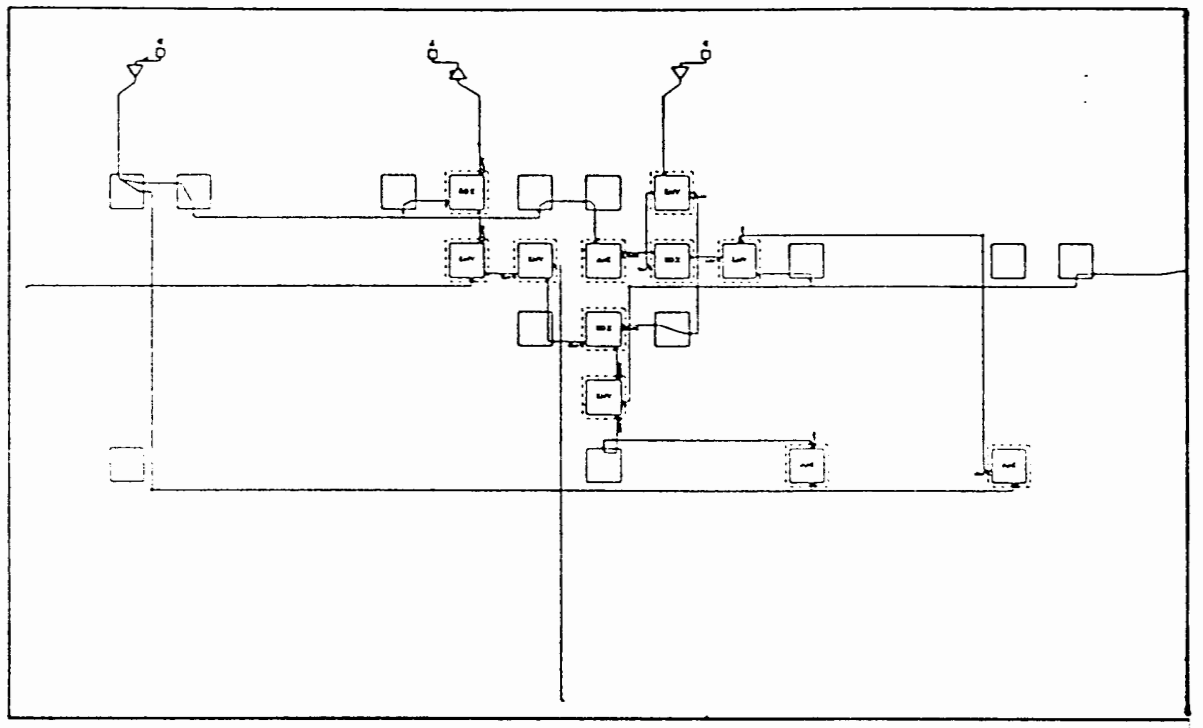

Figure A.5 Layout of MCNC f5 with ATMEL (IDS)

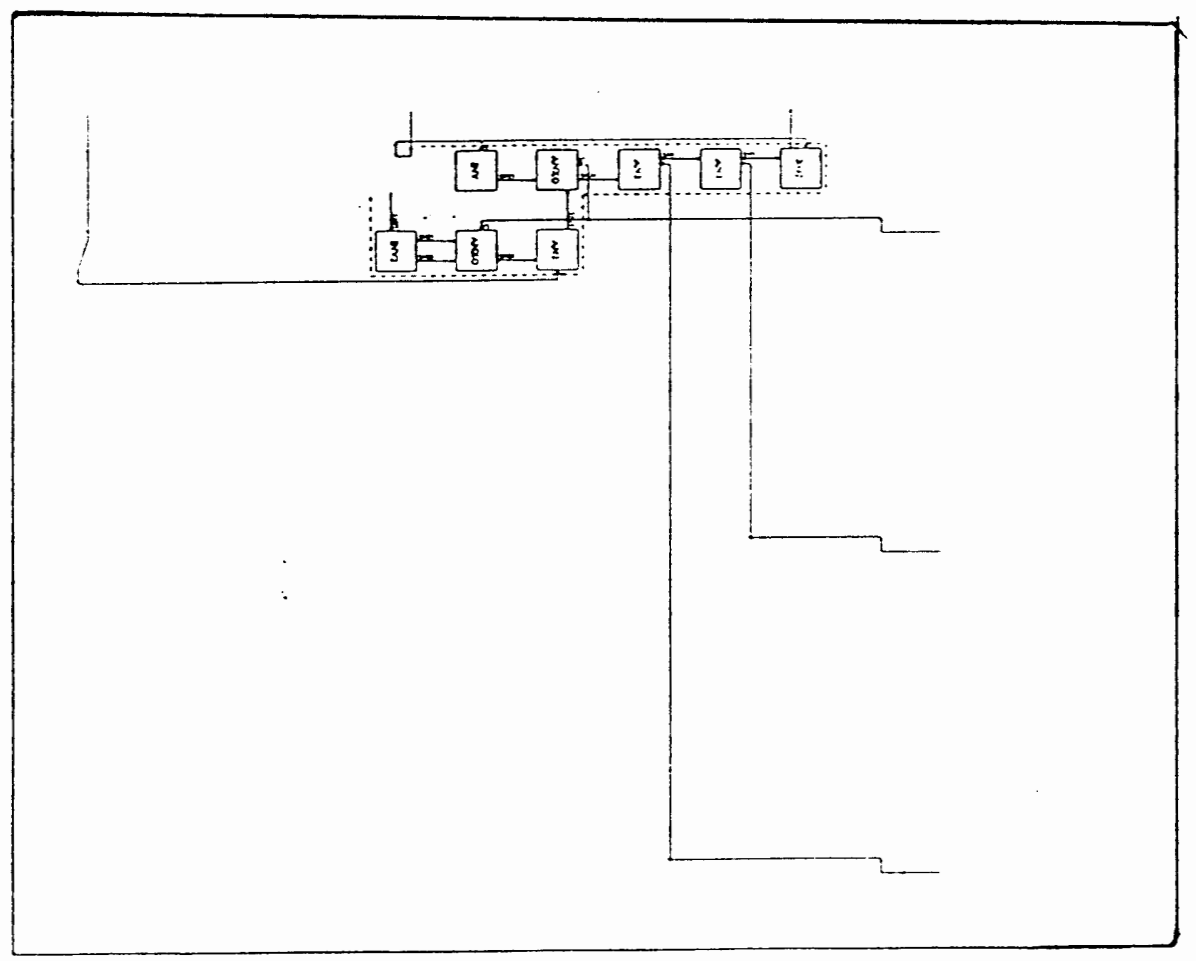

Figure A.6 Layout of MCNC f5 with TRM 


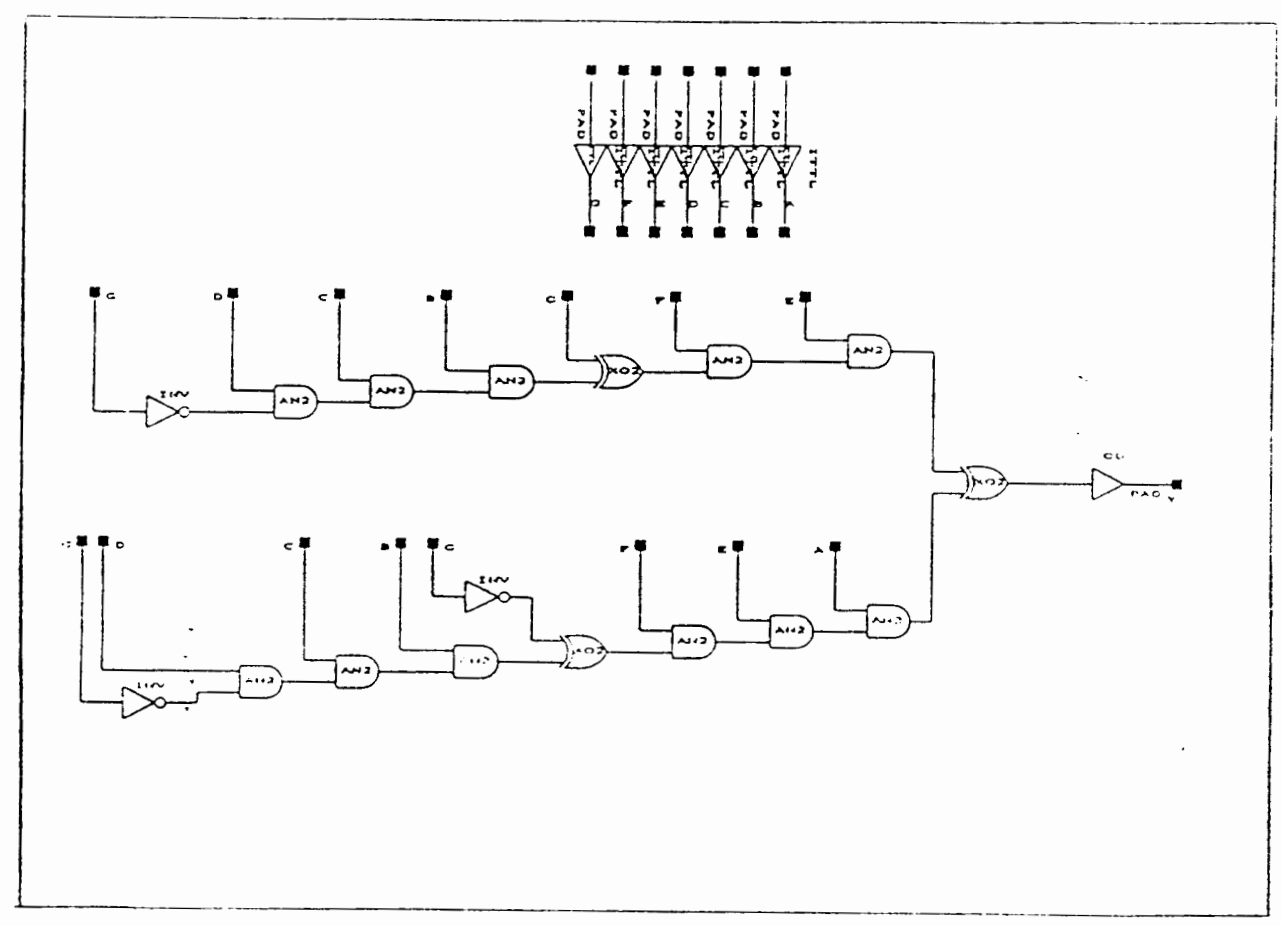

Figure A.1 ViewLogic Schematic of MCNC $5 \times 10$ 


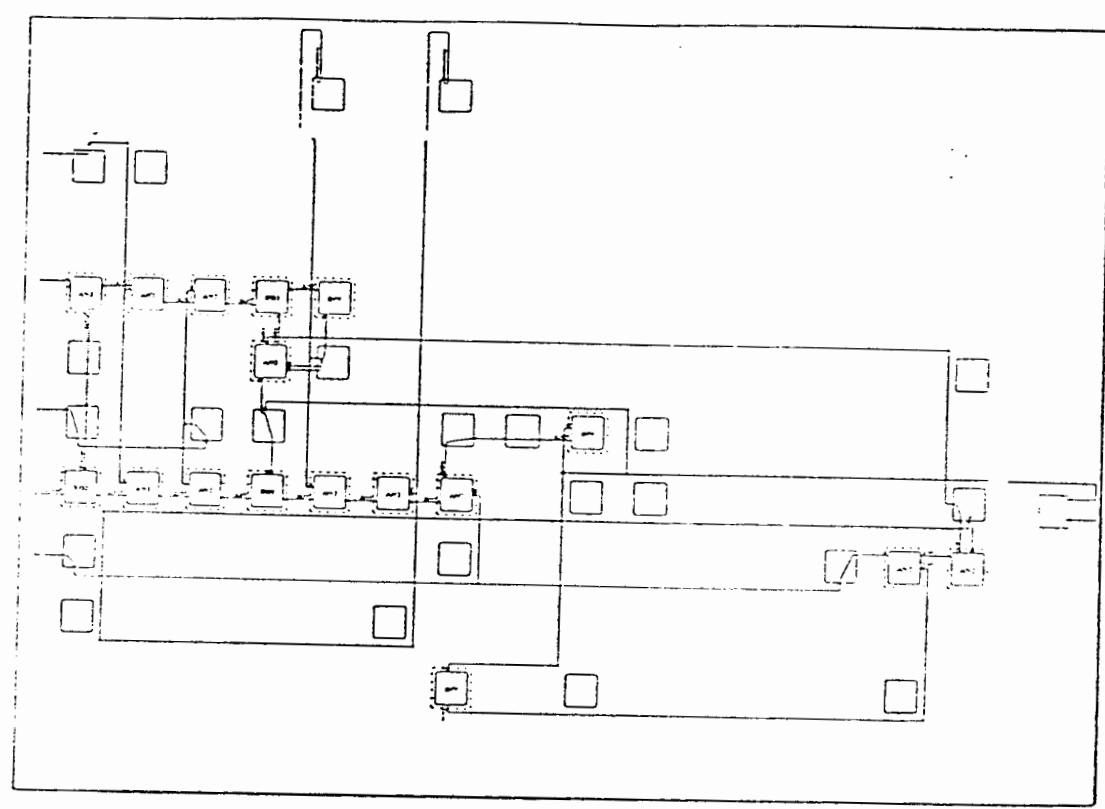

Figure A.2 Layout of MCNC 5x10 with ATMEL (IDS)

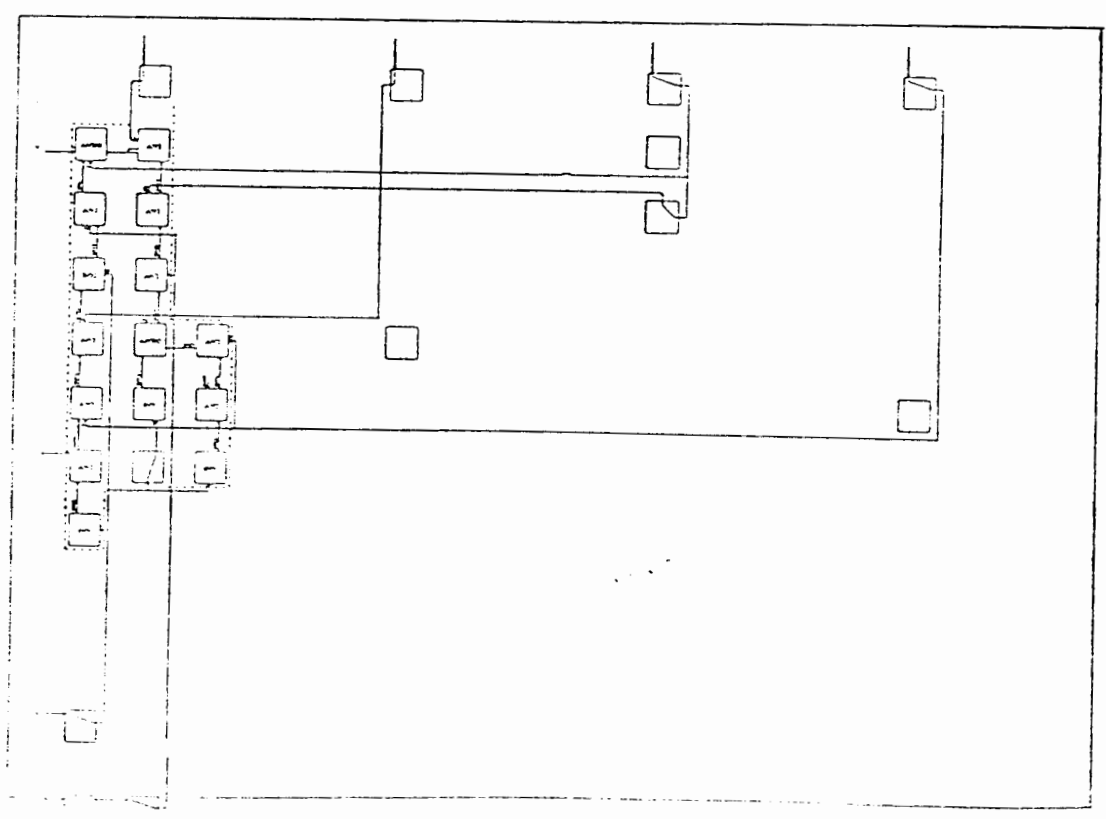

Figure A. 3 Layout of MCNC $5 \times 10$ with TRM 


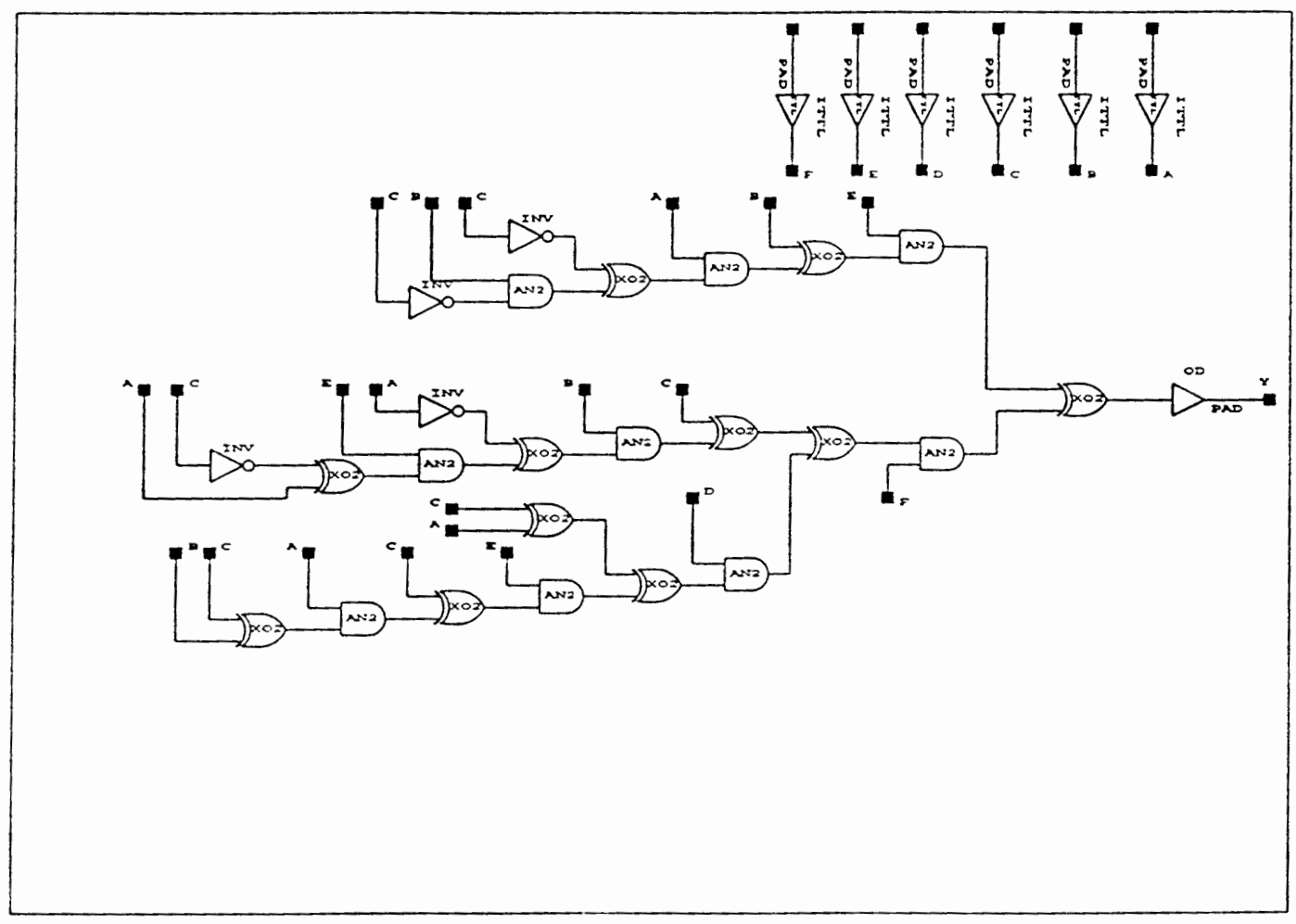

Figure A.7 ViewLogic Schematic of MCNC misex 54 


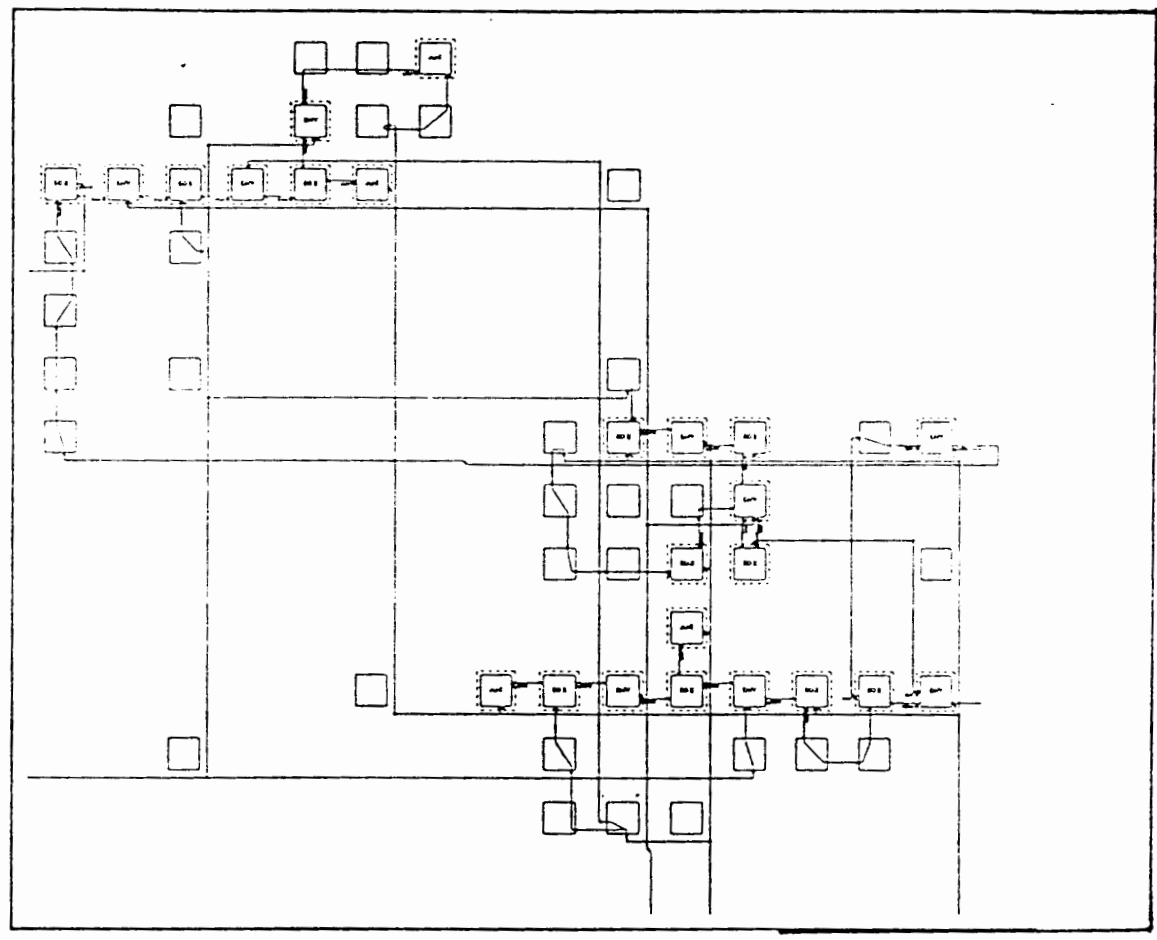

Figure A.8 Layout of MCNC misex 54 with ATMEL (IDS)

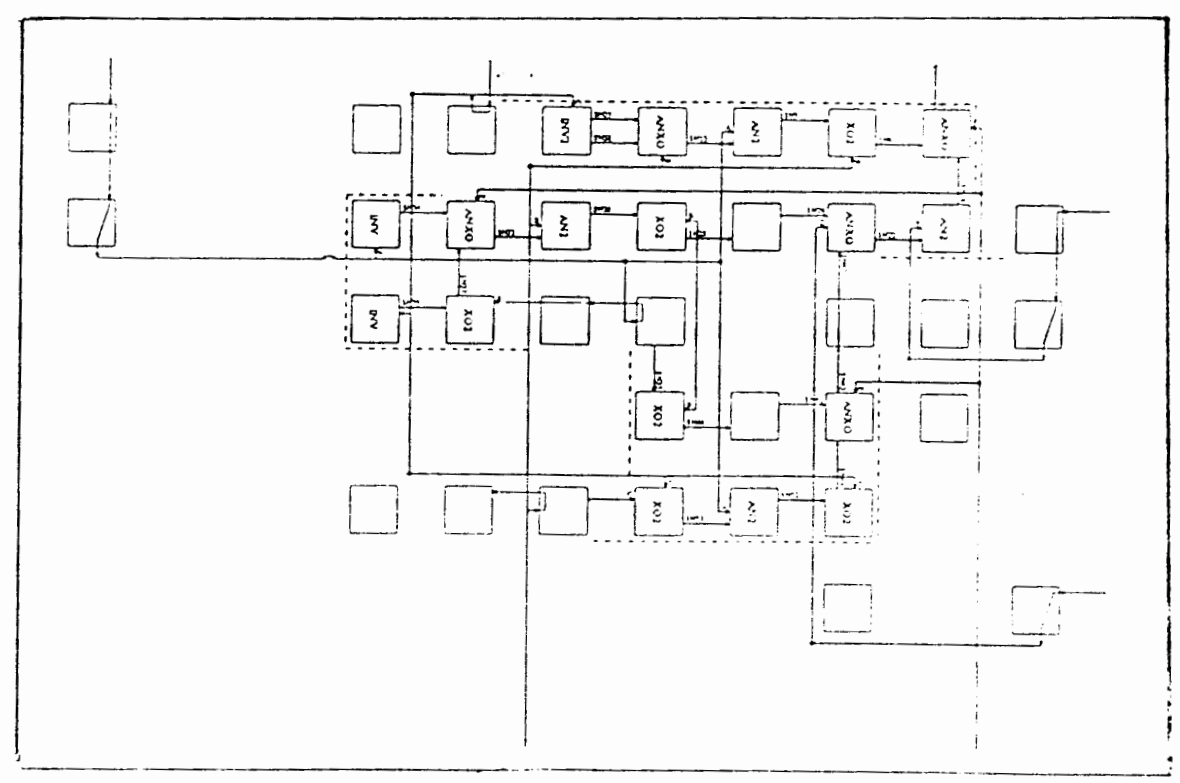

Figure A.9 Layout of MCNC misex.54 with TRM 
APPENDEX B

(Framework:TRM with VHDL/SCHEMATIC Design Entry) 


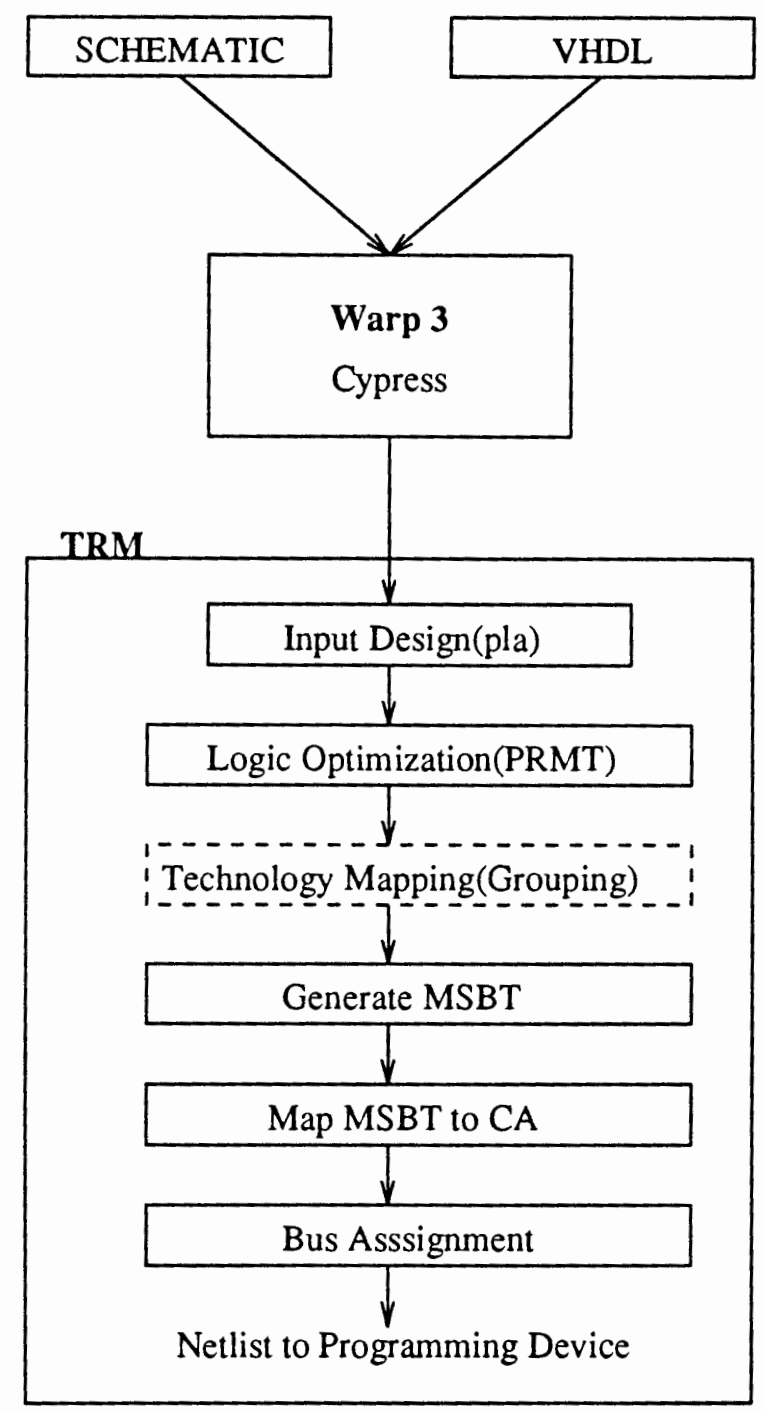

Figure B.1 Framework:TRM with VDHL/SCHEMATIC Design Entry

Warp3 is a trademark of Cypress Semiconductor 
VHDL description of 4 to 1 MUX (MUX41) with Enable.

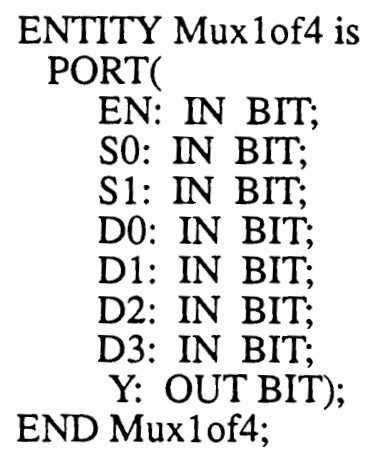

ARCHITECTURE unitsim of Mux 1 of 4 is

BEGIN

$\mathrm{Y}<=\mathrm{EN}$ AND ((D0 and not(S1) and not(S0))

$\mathrm{OR}$ (D1 and not(S1) and S0)

$\mathrm{OR}$ (D2 and $\mathrm{S} 1$ and not(S0))

OR (D3 and S1 and S0));

END unitsim;

The pla format output of the input VHDL design from Warp 3 VHDL based PLD synthesizer (Cypress Semicondutor).

i 7

.01

. $\mathrm{p} 4$

$1001-\cdots 1$

$110-1--1$

$101--1-1$

$111--11$

.$e$

Fig. B. 2 is the representation of the output netlist (blif format) form the program REMIT [1]. The grouped PRMT representation is shown in Fig. B.3 after the technology mapping phase. The final mapping with bus assignment for the MUX41 is shown in Fig. B.4. The layout implemention of the MUX41 is shown in Fig. B.5 [47]. 


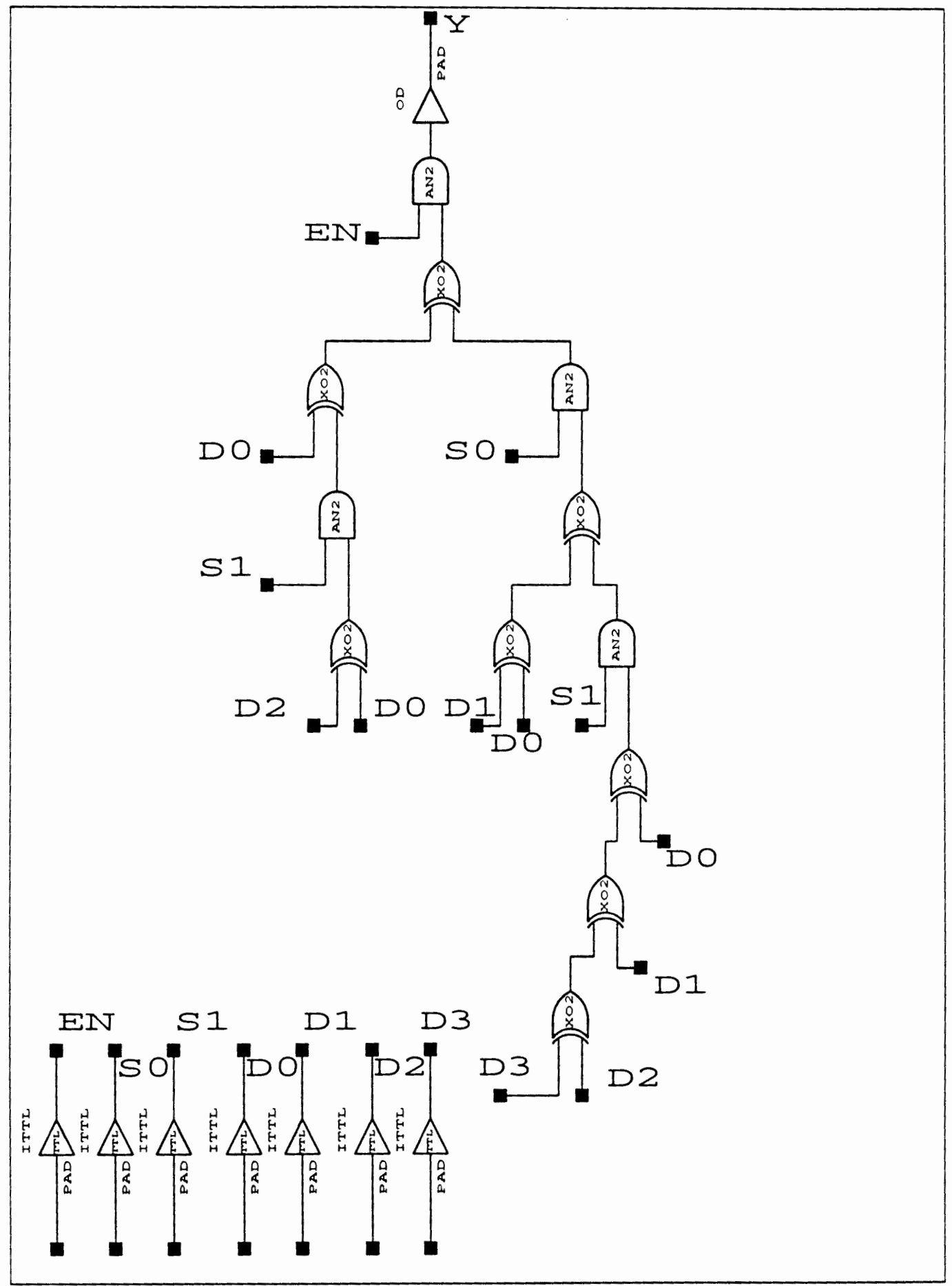

Figure B.2 PRMT Representation of MUX41 


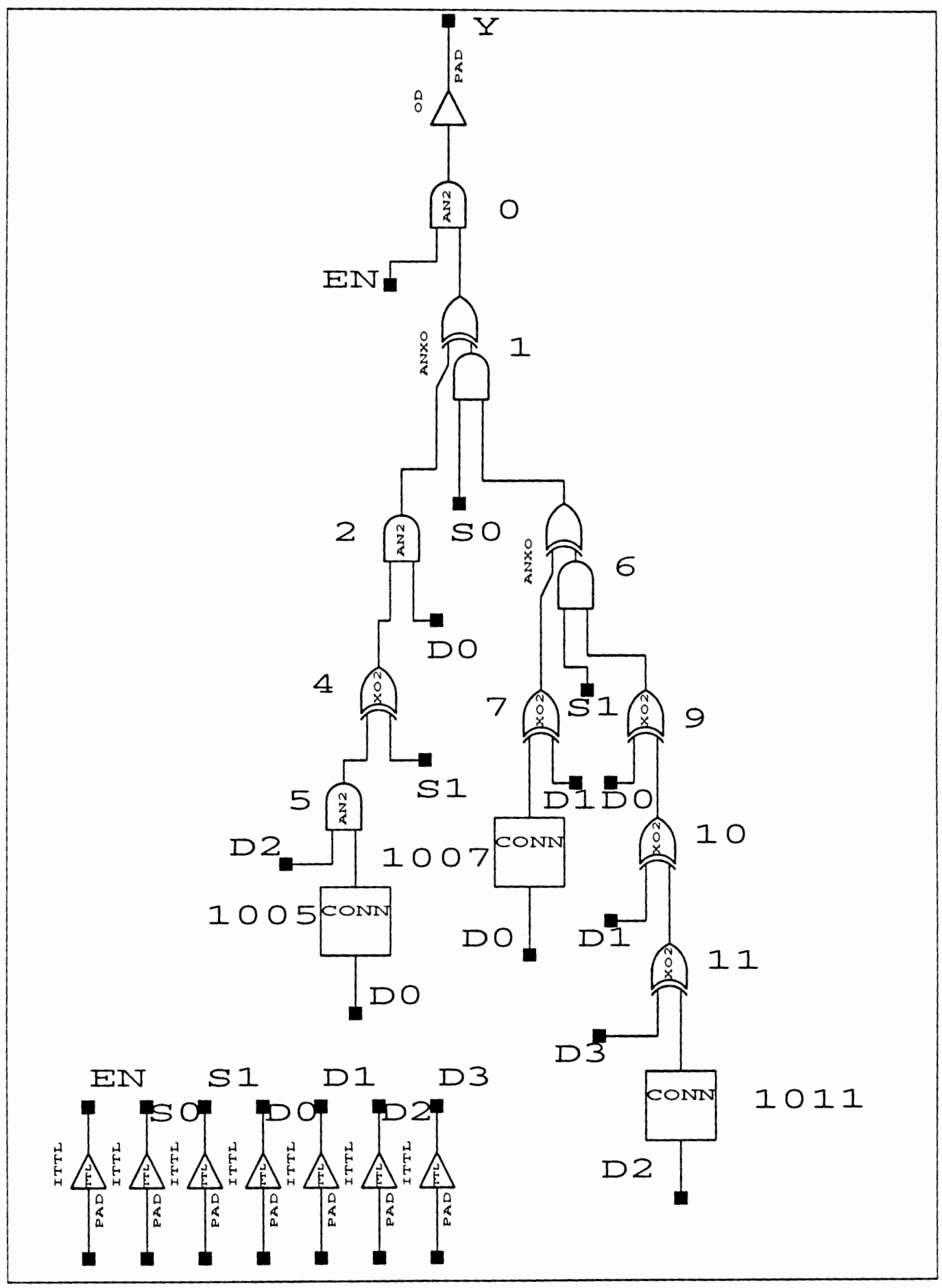

Figure B.3 Grouped PRMT Representation of MUX41 


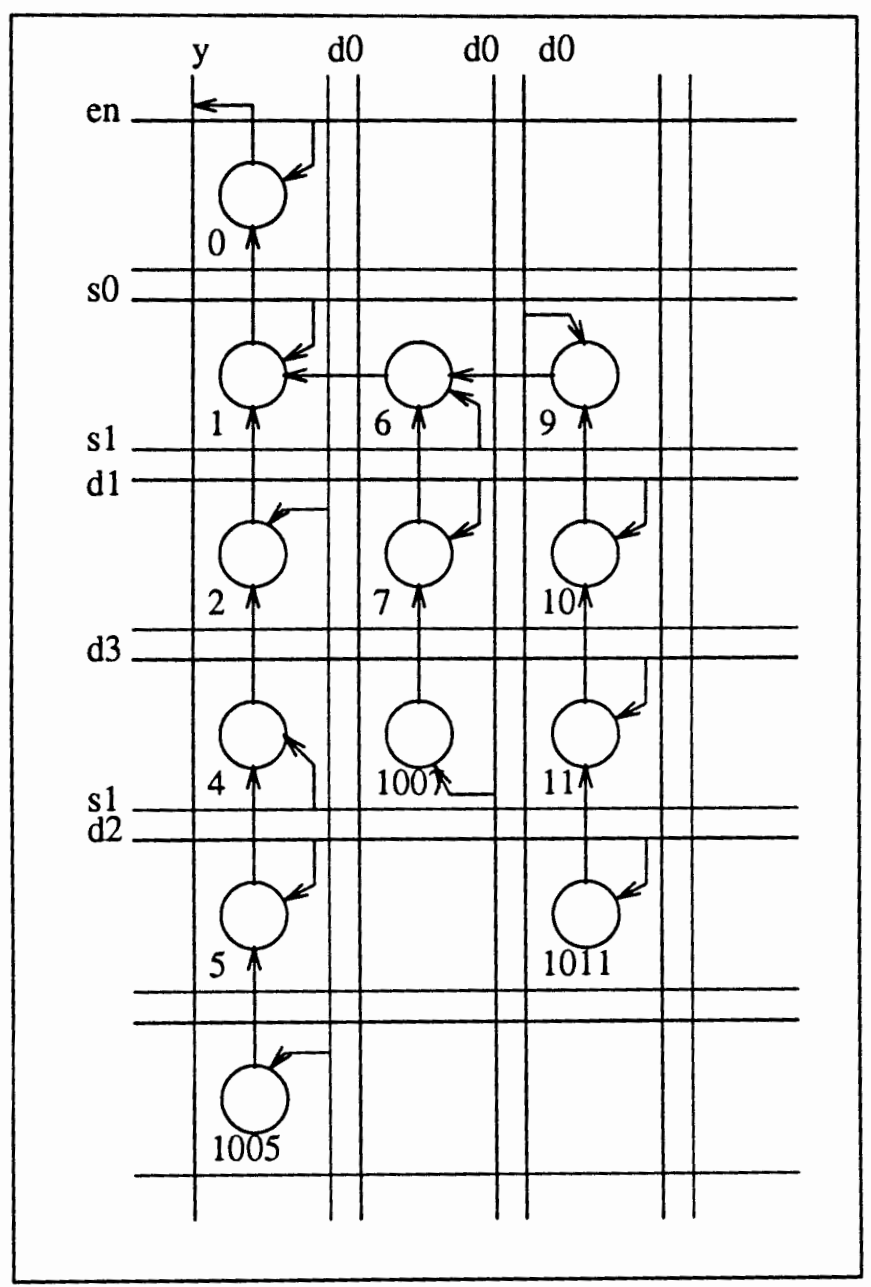

Figure B.4 MUX41 with Bus-Assignment

Comparing the Fig. B.4 and Fig. B.5; it is evident that the proposed Framework can produce good results. The MUX41 uses 10 cells for logic, 3 for wire and occupies a rectangular area of 18 with our TRM. Where as the ATMEL (IDS) [47] uses 13,5,20 cells for logic, wire and rectangular area respectively. In both the Figures, the cells needed for connecting to the buses were not included. 
MUX41 - 4-to-1 Multiplexer

\section{Layout for Shape 1}

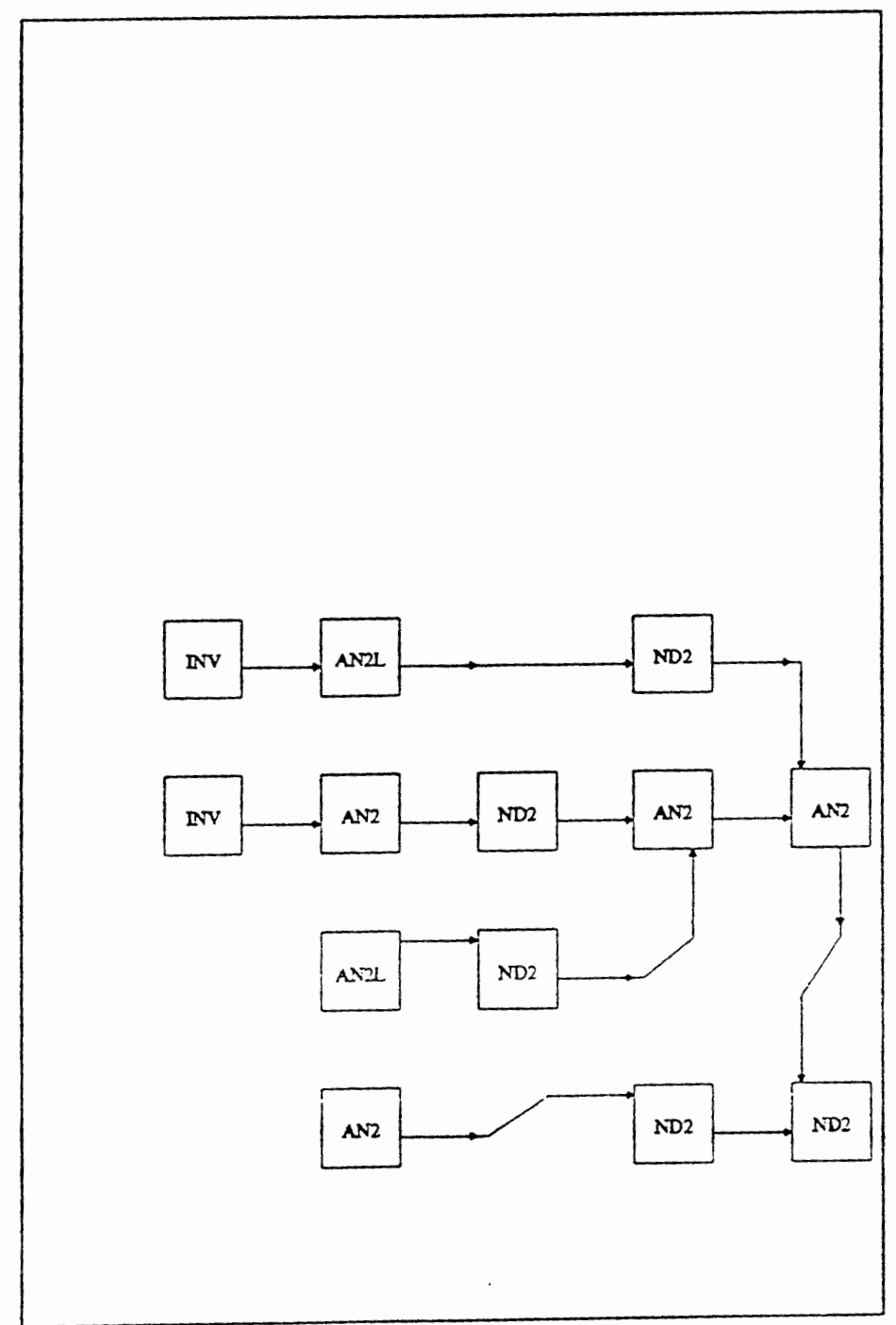

Figure B.5 MUX41 implementation in ATMEL (IDS) [47] 